\title{
A VISUAL SPREADSHEET USING HTML5 FOR WHOLE GENOME DISPLAY
}

\author{
NADA Alhirabi \\ A THESIS \\ IN \\ The Department \\ $\mathrm{OF}$ \\ Computer Science and Software Engineering \\ Presented in Partial Fulfillment of the Requirements \\ For the Degree of Master of Computer Science \\ CONCORDIA UNIVERSITY \\ Montréal, QuéBec, Canada
}

MARCH 2015

(C) Nada Alhirabi, 2015 


\section{CONCORDIA UNIVERSITY}

School of Graduate Studies

This is to certify that the thesis prepared

\section{By: $\quad$ Nada Alhirabi}

Entitled: $\quad$ A Visual Spreadsheet using HTML5 for Whole Genome Display

and submitted in partial fulfillment of the requirements for the degree of

\section{Master of Computer Science}

complies with the regulations of this University and meets the accepted standards with respect to originality and quality.

Signed by the final examining commitee:

Chair

Dr.Rajagopalan Jayakumar

Examiner

Dr.Juergen Rilling,

Examiner

Dr.Hovhannes Harutyunyan

Supervisor

Dr.Gregory Butler

Approved by

Chair of Department or Graduate Program Director

Dean of Faculty

Date 


\title{
Abstract
}

\section{A Visual Spreadsheet using HTML5 for Whole Genome Display}

\author{
Nada Alhirabi
}

Modern sequencing technology has enabled the cheap, rapid production of whole genomes. There is a need for visualization tools to show the data collected about a whole genome such as genes, proteins, annotations, and expression data. Many common approaches are developed such as the genome browser where sequence features are displayed as visual elements in tracks and features are aligned with their genome coordinates, visual networks where the data elements represented as nodes and relationship as edges, and traditional spreadsheet where each row captures the information about a gene/genome where the information is textual in nature, such as identifiers, descriptions, or sequences. Our study is focusing in the last approach with introducing some advanced features.

To build the system, the common used similar systems are reviewed, and during the implementation some software artifacts are reused such as reusing some JavaScript libraries to reduce the complexity of software development. Generally, an incremental method is used to develop the webpage starting from collecting the data from AspGD database, analyzing them, coding then testing them once at time. Our research group studies fungal genomes, so the spreadsheets are tested by displaying each of the Aspergilli genomes in the AspGD database (www.aspgd.org).

We have developed CGene and CGenome, pronounced See-Gene and See-Genome respectively, as a HTML5 web-based spreadsheets that can incorporate visual displays, as well as text, within the spreadsheet cells. Current displays use Scalable Vector Graphics (SVG) to present these spreadsheets which are generated from standard GFF3 files, standard output files from InterProScan, aspgd files from AspGD Gene Ontology Annotations File, and Chromosomal Feature File. All these files are analyzed to present them in a visual way that requires less effort to understand.

The main aim of our study is to take the advantages of the ability of humans to recognize patterns. The user can see the gene/genomes of interest as row-by-row of visualization. This can play powerful roll to ease the understanding of quantitive data by replacing them by graphical figures that make the comparison easier. 


\section{Acknowledgments}

I would like to express my sincere gratitude to my supervisor Dr.Greg Butler for his support, patience, knowledge and guidance throughout my period of my research.

I am particularly grateful to my beloved husband Majed for being supportive during the hardest moments, my daughter Jood for giving me the warmest smile every day, and to my baby Lujain for sharing the most stressful moments with me. Many thanks to them for sharing with me this experience by its good and ugly parts.

I owe my deepest gratitude to my mother Fawziah and my father Abdullah, whom I miss a lot, for their unconditional love, support and encouragement for every step in my life. Thanks to my family: Majed, Muhammed, Hessah, Munerah and Abdulaziz for their nice words that encouraged me to follow my dreams. Thanks to my grandmother for her warm words and tears every time she called me.

Many thanks go to the friendly lab mates at Concordia University: Asma Mistadi, Christine Kehyayan, Faizah Aplop, Lin Cheng, Patricia Hanney and Stuart Thiel for their help during my research. 


\section{Contents}

Abstract

\begin{tabular}{|ll} 
Acknowledgments & iv
\end{tabular}

List of Figures viii

List of Tables $\quad x$

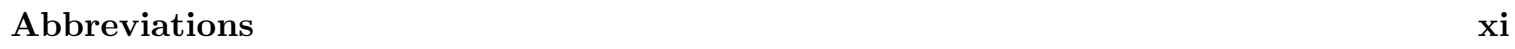

$\begin{array}{lll}1 & \text { Introduction } & 1\end{array}$

1.1 Visual Spreadsheet for Whole Genome $\ldots \ldots \ldots$. . . . . . . . . . . . . . . . . . 1

1.1 .1 What is Data Visualization, and Why Do It? . . . . . . . . . . . 1

1.1 .2 Genomics Sequencing and Annotation . . . . . . . . . . . . . . 3

1.1.3 Whole Genome Visualization Tools . . . . . . . . . . . . . . . . . . . 3

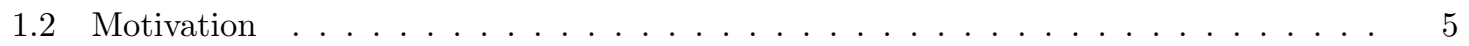

1.3 Research Challenges $\ldots \ldots \ldots \ldots \ldots \ldots \ldots \ldots$

1.4 Research Contributions $\ldots \ldots \ldots \ldots \ldots \ldots \ldots$

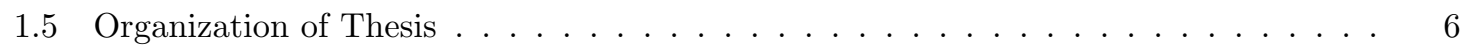

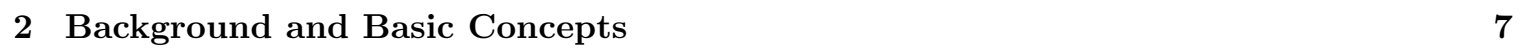

$2.1 \quad$ Basic Concepts of Biology $\ldots \ldots \ldots \ldots \ldots \ldots \ldots \ldots$

2.1 .1 Cell, Nucleic Acids and Amino Acids . . . . . . . . . . . . . 7

$2.1 .2 \quad$ Central Dogma of Molecular Biology . . . . . . . . . . . . . . . . . . 8

2.2 Gene and Gene Model . . . . . . . . . . . . . . . . . . . . . . . . . . 10

$2.2 .1 \quad$ Genes, Exons, Introns and Coding Segments _ . . . . . . . . . . . . . 10

2.2 .2 Gene Model . . . . . . . . . . . . . . . . . . . . . . . . 10

$2.3 \quad$ Gene Annotation and Gene Ontology . . . . . . . . . . . . . . . . . . . . 11

2.3 .1 Gene Annotation . . . . . . . . . . . . . . . . . . . . 11

2.3 .2 Gene Ontology $\ldots \ldots \ldots \ldots$

2.3 .3 Go Slims . . . . . . . . . . . . . . . . . . . . . . . . . . 16

2.4 Protein Structure, Protein Domain and its Architecture . . . . . . . . . . . . . . 16

$2.4 .1 \quad$ Protein Structures $\ldots \ldots \ldots \ldots$. . . . . . . . . . . . . . . . . . . . 16 
2.4 .2 Protein Domains . . . . . . . . . . . . . . . . . . . . . . . . . . . . . . . . . . .

$2.4 .3 \quad$ Protein Domains Architecture . . . . . . . . . . . . . . . . . . . . . . . . . 19

2.5 Principles of Biological Data Visualization $\ldots \ldots \ldots \ldots \ldots$

2.5 .1 Visual Representation Understanding . . . . . . . . . . . . . . . . . . . . . . . . . . . . . . . . . .

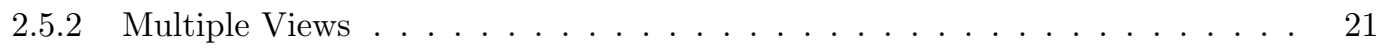

2.6 Whole Genome Visualization Tools $\ldots \ldots \ldots \ldots \ldots$

2.6 .1 Genome Browsers . . . . . . . . . . . . . . . . . . . 26

2.6 .2 Textual Spreadsheet $\ldots \ldots \ldots \ldots$. . . . . . . . . . . . . . . . . . 27

2.6 .3 Network Visualization Tools . . . . . . . . . . . . . . . . . . . . . . . 28

2.6 .4 Tree Layout Visualization Tools $\ldots \ldots \ldots$. . . . . . . . . . . . . 30

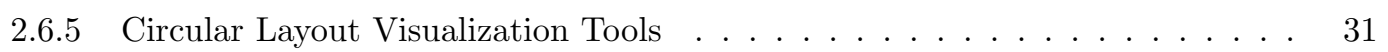

2.6 .6 Related Works . . . . . . . . . . . . . . . . . . . . . . . . . 32

3 CGene and CGenome: System Analysis and Design 35

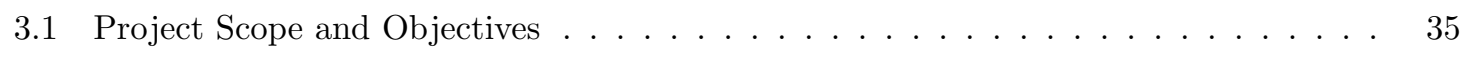

3.2 System Activity Diagram $\ldots \ldots \ldots \ldots \ldots$

3.3 General Requirements $\ldots \ldots \ldots \ldots \ldots \ldots \ldots$

3.3 .1 Functional Requirements $\ldots \ldots \ldots \ldots \ldots \ldots$

3.4 Domain Model $\ldots \ldots \ldots \ldots \ldots \ldots$

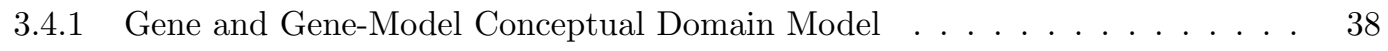

$3.4 .2 \quad$ Protein and Protein-domain Architecture Conceptual Domain Model . . . . . 39

3.5 Data Sources and Formats . . . . . . . . . . . . . . . . . . . . . . . 43

3.5 .1 Data Selection . . . . . . . . . . . . . . . . . . . . . . . . . . . . . . . . . . 43

3.5 .2 Data Formats . . . . . . . . . . . . . . . . . 54

3.6 Data File Preprocessing $\ldots \ldots \ldots \ldots \ldots \ldots \ldots \ldots \ldots$

3.7 Why HTML5 and SVG? . . . . . . . . . . . . . . . . . . . . . . . . . . . . . . . . 62

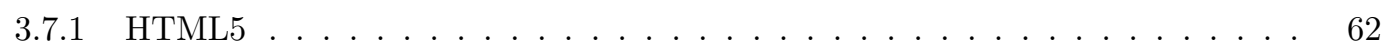

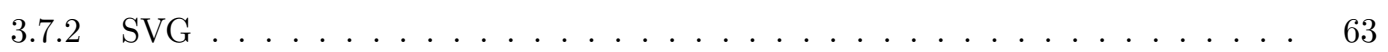

3.7 .3 JavaScript . . . . . . . . . . . . . . . . . . . . . . . 67

3.7 .4 Why HTML 5 , SVG and JavaScript? . . . . . . . . . . . . . . . 68

3.8 Graphical User Interface $\ldots \ldots \ldots \ldots$. . . . . . . . . . . . . . . . . . . 69

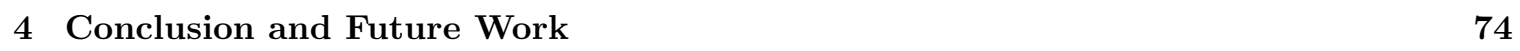

4.1 Summary of Works . . . . . . . . . . . . . . . . . . . . . . . . 74

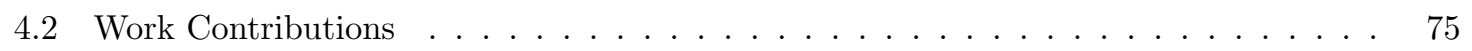

4.3 Research Limitations and Future Work . . . . . . . . . . . . . . . . . . 75

4.4 Resource of the system . . . . . . . . . . . . . . . . . . . . 79

\begin{tabular}{ll}
\hline Bibliography & 79
\end{tabular}

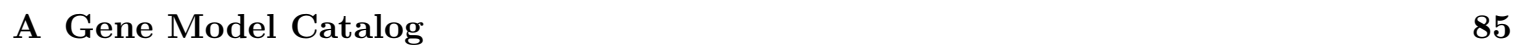


\begin{tabular}{ll}
\hline B Protein Domain Catalog & 98
\end{tabular}

\begin{tabular}{ll}
\hline C Comparative Genomics & 114
\end{tabular}

\begin{tabular}{ll}
\hline D Glossary & 115
\end{tabular} 


\section{List of Figures}

1 Tabular view for fungus $A_{-} a c i d u s_{-} C B S_{-} 106.47$ features. . . . . . . . . . . . . . 2

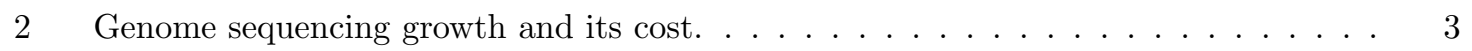

3 Illustration of chromosome that have DNA double helix stranded molecule. . . . . . 8

$4 \quad$ A summary of Central Dogma of Molecular Biology $\ldots \ldots \ldots$. . . . . . . . . . . 9

5 Removal of introns during the splicing process to produce a mature mRNA in eukary-

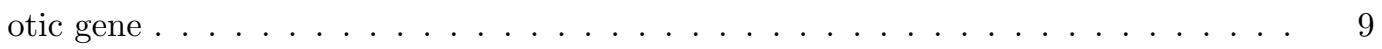

$6 \quad$ Gene model in general eukaryotic gene and A acidus CBS 106.47 fungi in CGene

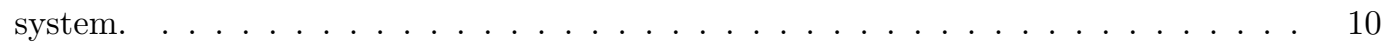

7 Graphs illustrate the increased amount of Genome Sequencing Projects and annotation. 11

8 Generalized flow chart illustrates Genome Annotation procedure. . . . . . . . . . . . . 12

$9 \quad$ Graph shows regulates and part_of relationships between Molecular Function and Biological Process. . . . . . . . . . . . . . . . . . . . . . . . 14

$10 \quad$ Gene Ontology terms and annotations from Quick $G O$ web browser. . . . . . . . . . 15

11 3D crystal structure of Endophilin BAR domain with the domain architecture repre-

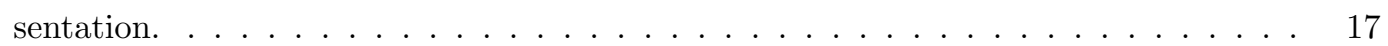

12 Domain architecture representation for DNA repair helicase UVH6 in different DBs. 19

13 Illustration of A Multi-Level Typology of Abstract Visualization Tasks . . . . . . . . 20

14 A summary illustration of dataset types and their types. . . . . . . . . . . . . 21

15 MulteeSum: an example of Multiple-View system. . . . . . . . . . . . . . . . 22

16 Model-View-Controller (MVC) architecture. . . . . . . . . . . . . . . . . . 25

17 Rat genome on UCSC Genome Browser . . . . . . . . . . . . . . . . . . . . . . . . . . . . . .

18 ISA-TAB representation tool $\ldots \ldots \ldots \ldots \ldots \ldots \ldots \ldots$

19 Visualization of protein interaction networks in Cytoscape. . . . . . . . . . . . . 29

20 Representing phylogenetic tree in iTOL $\ldots \ldots \ldots \ldots \ldots \ldots$

21 Using Circos Circular layout to express genomic annotation data. . . . . . . . . . . . 31

22 Screenshot of FancyGene website $\ldots \ldots \ldots \ldots \ldots \ldots$

23 FeatureStack showing RFX gene family members over a diverse set of species . . . . 34

$24 \quad$ Activity diagram for visual HTML5 spreadsheets system: CGene and CGenome. . . 37

25 Gene-Model domain conceptual class diagram. . . . . . . . . . . . . . . . 41

$26 \quad$ Protein and Protein-domain conceptual class diagram. . . . . . . . . . . . . . . . 42 
27 Pareto chart shows InterPro entries with their frequencies in the five most common filamentous fungi of the genus Aspergillus. . . . . . . . . . . . . . . . . . . 44

28 Pareto chart shows the top 25 selected InterPro domains in the five most common filamentous fungi of the genus Aspergillus . . . . . . . . . . . . . . . . 46

29 Chart shows Molecular Function Go Slims Aspergillus occurrence percentage in the

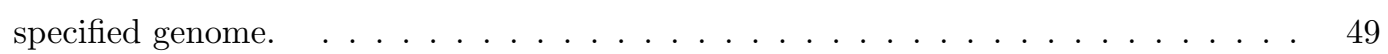

$30 \quad$ Chart shows Cellular Component Go Slims Aspergillus occurrence percentage in the

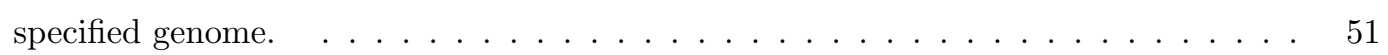

31 Chart shows Biological Process Go Slims Aspergillus occurrence percentage in the

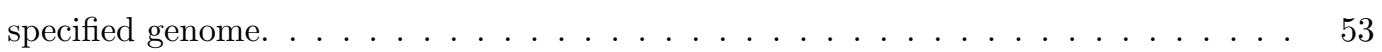

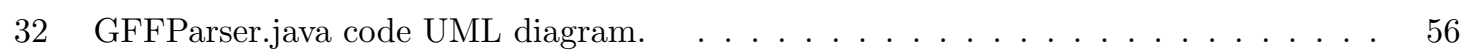

$33 \quad$ ProteinDomainParser.java code UML diagram. . . . . . . . . . . . . . . . . . . . 57

34 GeneProteinLinking.java code UML diagram. . . . . . . . . . . . . . . . . . . . . . . . . . . . .

35 GeneModelSVG.java code UML diagram. . . . . . . . . . . . . . . . . . . 59

36 DomainArchitectureSVG.java code UML diagram. . . . . . . . . . . . . . . . 60

37 ChromosomeLocationParser.java code UML diagram. . . . . . . . . . . . . . . . . 61

38 CreatHTMLtable.java code UML diagram. . . . . . . . . . . . . . . . . . 61

39 Illustration of the effect of magnification on vector graphics versus raster graphics. . 64

$40 \quad$ Vector graphics concept illustration. . . . . . . . . . . . . . . . . . . . 64

41 Basic vector shapes. . . . . . . . . . . . . . . . . . . . . . . . 65

$42 \quad$ SVG image for gene locations in chromosomes as part of CGenome spreadsheet. . . . $\quad 67$

43 Master page of the visual spreadsheet system using HTML5 for the whole genome. . $\quad 70$

44 CGene spreadsheet with normalized SVG images $\ldots \ldots \ldots \ldots \ldots \ldots$. . . . . . 71

45 CGene spreadsheet with unnormalized SVG images . . . . . . . . . . . . . . 71

46 Snapshot of CGene spreadsheet. . . . . . . . . . . . . . . . . . . 72

47 CGenome spreadsheet for different genomes comparative analysis. . . . . . . . 73 


\section{List of Tables}

$1 \quad$ Proteins' 20 amino acids . . . . . . . . . . . . . . . . . . . . . . . . . . . . . . . . 8

2 The status of Gene Ontology as September 2007 and September 2009. . . . . . . . . 15

$3 \quad$ The top 25 selected InterPro domains information . . . . . . . . . . . . . . . . . . . 45

4 Table shows Molecular Function Go Slims Aspergillus occurrence in specified genomes: A. niger, A. nidulans, A. fumigatus and A. oryzae. . . . . . . . . . . . . . . . . . . . 48 48

5 Table shows Cellular Component Go Slims Aspergillus occurrence in specified genomes: A. niger, A. nidulans, A. fumigatus and A. oryzae. . . . . . . . . . . . . . . . . . . 50

6 Table shows Biological Process Go Slims Aspergillus occurrence in specified genomes: A. niger, A. nidulans, A. fumigatus and A. oryzae. . . . . . . . . . . . . . . . . . . . 52

$7 \quad$ Catalog of visualization tools for Gene Models . . . . . . . . . . . . . . . . . . . . . 86

8 Catalog of tabular view for Gene Annotations . . . . . . . . . . . . . . . . . . . . . . 90

$9 \quad$ Different glyph's visualization for Gene Models . . . . . . . . . . . . . . . . . . . . . 91

10 Catalog of visualization tools for Protein Domain Architecture. . . . . . . . . . . . . 99

11 Different glyph's for Protein Domain Architecture . . . . . . . . . . . . . . . . . . . 103

12 Pfam Domain Architecture graphics. . . . . . . . . . . . . . . . . . . . . . . . . . . . 107

13 DOG: Protein Domain Structure Visualization . . . . . . . . . . . . . . . . . . . . . 111

14 Comparative Genomics catalog . . . . . . . . . . . . . . . . . . . . . . . . . . . . . . 114 


\title{
Abbreviations
}

\author{
BED . . . . B Browser Extensible Data format \\ CMV ...... Coordinated and Multiple Views \\ EV ...... Exploratory visualization \\ GFF ...... General Feature Format \\ GO . . . . . . G Gene Ontology \\ HTML . . . . . . HyperText Markup Language \\ HMM . . . . . . Hidden Markov Models \\ ISA-Tab . . . . Investigation-Study-Assay Tab-delimited \\ MSA . . . . . multiple sequence alignments \\ SVG ...... Scalable Vector Graphics \\ Vis ....... Visualization \\ W3C ...... World Wide Web Consortium
}




\section{Chapter 1}

\section{Introduction}

\subsection{Visual Spreadsheet for Whole Genome}

\subsubsection{What is Data Visualization, and Why Do It?}

Information overload (infobesity) is considered as one of the major human-computer communication problems. Many efforts were presented to solve this problem, and one of them is data visualization. The main benefit of using this solution is enabling the users to explore and explain data better by taking the advantage of the human ability to recognize patterns O'Donoghue et al., 2010]. Data visualization, as defined by Latham and Latham, 1995], is the process of "representing data as visual image". The created images are combination of graphics primitives such as lines and triangles accompanied by attributes such as shades and colours that used to represent different quantities measurements Tufte and Graves-Morris, 1983].

When we are given data, the first question is how to design a system to visualize this kind of data. However, we previously need to think deeply about what does this data mean in the real world and what is the type of this data. For example, if the data given is $\{$ Apple, Banana\}, many questions about the meaning will be guessed such as do they mean fruit names or company names Munzner, 2014. The semantic is very crucial in this matter in order to see how to group or organize this data. In addition to the semantic, the structural or mathematical data type is important to perform visualization analysis. There are different categories for the data type of what can be visualized. One of the categorization divides the dataset types into four basic types that are tables, networks, fields and geometries. Each one of them has a combination of associated properties such as attributes, links, items, positions and grid cells Munzner, 2014.

In our work, we are focusing on producing spreadsheets which is a tabular display method used frequently to organize and present a high-level summary of annotated genomic information Dudley and Karczewski, 2013. In general, a table as display is a combination of rows and columns and their intersection is a single cell. As a dataset, a simple table as seen in Figure 1, consists of items (rows), attributes (columns) and their intersection is a value (cell). Complex/multidimensional tables will have more complex cell indexing with multiple keys Munzner, 2014. 


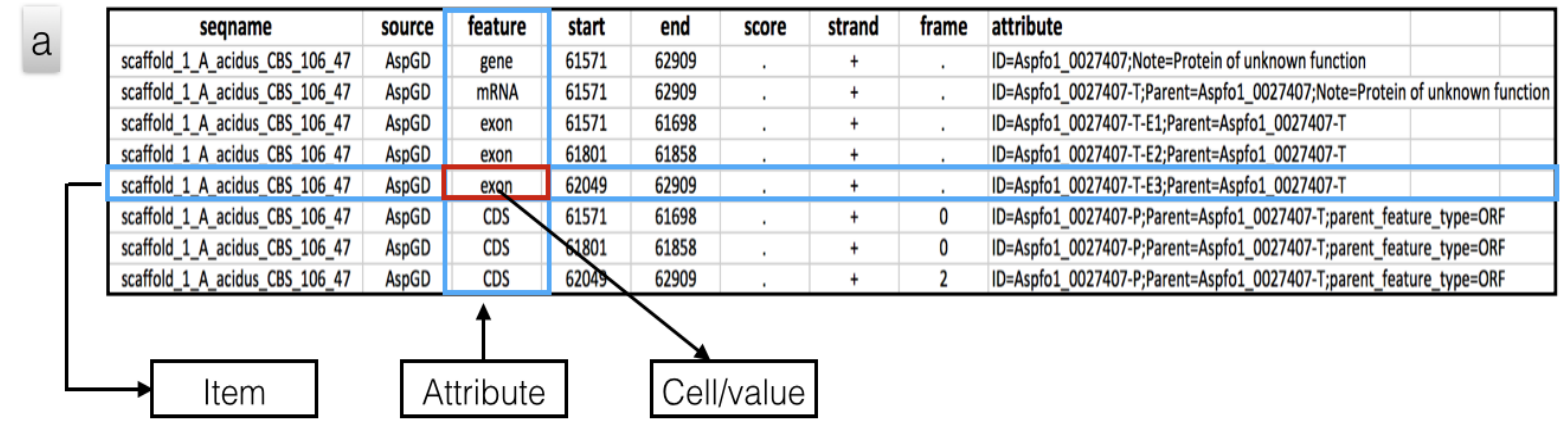

\begin{tabular}{l|l|l|}
\hline$\square$ & $\begin{array}{l}\text { Gene ID } \\
\text { length } \\
\text { Aspfo1_0027407 }\end{array}$ & Gene model \\
\hline$\square$ & $\mathbf{1 3 8}$
\end{tabular}

Figure 1: Tabular view for fungus $A_{-} a c i d u s_{-} C B S_{-} 106.47$ features; where items represented by rows, attributes represented by columns and their intersection is a value which represented by cell. Table (a) represents the features in simple tabular veiw with textual format fom $A_{-}$acidus_CBS_106.47 GFF file. Table (b) represents the same information but in visual and textual format as produced by our system called $\boldsymbol{C G e n e . ~}$

During the design of data visual representation, the scientists may spend minutes or hours to create the visual display manually. However, in some points their ability to draw by hand will be impossible especially with a massive data such as biological datasets. In this point, the computerbased system is required to save the human effort compared to the manual way Munzner, 2014. The computer-based data visualization systems have the ability to demonstrate the datasets in interactive visual representation. This representation is used to extend the understanding and help users to make decision effectively Card et al., 1999].

According to Munzner, 2014, data visualization is used in many situations. Visualization is mainly used in a situation when a human assessment and evaluation is needed to make decisions rather than depending on computational decision-making. When users do not know the exact questions to ask about the data and they need to explore things, visualization in this case is also required. In addition, visualization is needed when the experts know something and they want to explain it to others visually or to help them to assess the data easily. In some situations, the need for machine learning and computational techniques for making decisions is high. For example, if scientists know the questions about the dataset very well, but they want to make sure if the outcome from the system is as they expected or not. In that case, they use machine learning techniques to make decisions. 


\subsubsection{Genomics Sequencing and Annotation}

Genomics is a scientific discipline that deals with sequencing, and analyzing genomes Hrmova and Fincher, 2009. The main goal of genomics is to identify an organism's complete DNA sequence, including all of its genes. This organism can an animal, a fungus, a plant, a virus etc.

Today, genome sequencing is growing fast and its cost is dropping at the same time as we see in Figure 2. Modern sequencing technology has enabled an inexpensive, rapid production of whole genomes. Million of genomes such as human genome has been sequenced and over 2.7 billion bases are available in public databases Kent et al., 2002.

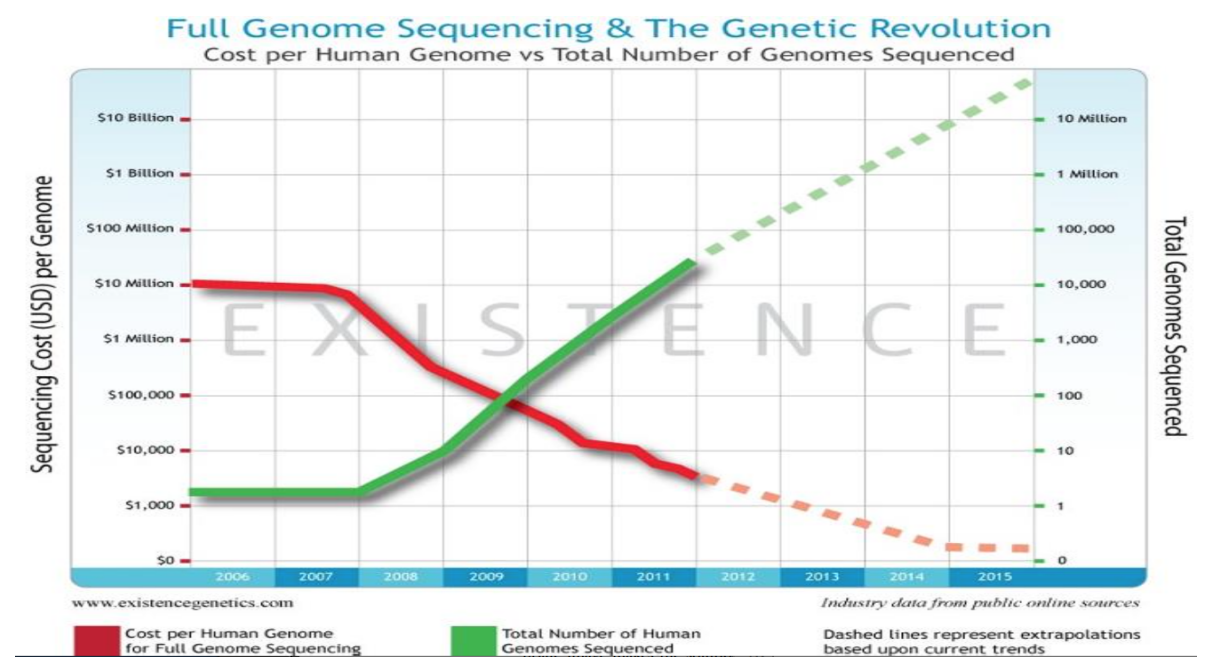

Figure 2: This chart illiterate that the number of genomes has speedily increased, while the costs of sequencing a whole human genome has decreased.Credit: How DNA sequencing works and how it became affordable (http://www.extremetech.com/extreme/151133-the-quest-for-the-1000genome/2)

Once a genome is sequenced, there is a need to understand what these sequences mean since each genome has all the information required for building and maintaining that organism. The process of attaching identities and functions to sequences is called genome annotation. As a result of genome annotation, massive amount of data are recorded in several databases; and understanding this huge amount of genomic data is quite a challenge Nielsen et al., 2010. To be focused, mostly scientists prefer genome assessment at the level of an exon, a gene, and a biochemical pathway according to Kent et al., 2002. The main purpose of this assessment is to find the key features of the genome, particularly the genes and their products Stein, 2001].

\subsubsection{Whole Genome Visualization Tools}

With the exponential and rapid development of genomic data, the need for visualization tools for presenting genome annotation is becoming more pressing. As stated previously, scientists mostly prefer genome assessment at the level of an exon, a gene, and a biochemical pathway Kent et al., 
2002]. Consequently, there is a need for tools for the visualization of the data collected about a whole genome such as genes, proteins, annotations, and expression data.

As we mention in the previous section that the dataset representation is divided into four basic types that are tables, networks, fields and geometries. There are some common approaches that used this categorization for the visualization purposes such as genome browsers, spreadsheets, trees and networks visualization tools.

Genome browsers are valuable tools for many purposes such as identifying genes, locating genes, and observing their detailed information in the genomic context. In these browsers, the sequence features are displayed as visual elements in tracks and features are aligned with their genome coordinates. For each view, users can display part of the genome and zoom in and out to control the amount of the displayed data such in UCSC browser (http://genome.ucsc.edu) Kent et al., 2002. Ensembl (http://www.ensembl.org), JBrowse (http://jbrowse.org), and GBrowse (http://www.gbrowse.org) are some of the most common browsers used to visualize genomic data.

Tree layout is commonly used in the applications that need an efficient way to present the phylogenetic context. By using the tree layout, a better understanding for organism evolution patterns in gene and genome can be achieved [Soltis and Soltis, 2003]. Interactive Tree Of Life (ITOL) (http://itol.embl.de) is one of the well-known systems adapted this kind of visualization where the phylogenetic tree is displayed as rooted, unrooted, or circular tree.

With massive datasets, the interpretation and analysis of relationships between biological molecules becomes a big challenge. One of the best ways to express these relations is using networks where nodes (circles) represents the elements and the edges represent the interaction between them Pavlopoulos et al., 2008. These relationships could be physical relationships such as protein interaction, gene-gene interaction, having a shared protein domain or family, regulatory transcription factors that regulate the genes, and functional interaction. Today, there are many 2D graph visualization tools that used to visualize biological interactions. These tools are varying on their user friendliness especially when thousands of circles with many connections have to be visualized and explored Pavlopoulos et al., 2008. One of the most common network visualization tools these days is Cytoscape (http://www.cytoscape.org) which is an open source network visualization and analysis tool for bioinformatics. Cytoscape enables users to display bioinformatic networks through the web with the ability to visualize a large-scale network that has thousands of nodes and edges with a variety of node shapes and colors Pavlopoulos et al., 2008 Gehlenborg et al., 2010.

Spreadsheets is another way to represent the data, in which the information is textual in nature. Each row captures the information about a gene/genome such as identifiers, descriptions, or sequences. For tabular (or spreadsheet) data representation in biology, there are some efforts that are done. Most of the efforts that are done was to find a universal format to exchange omics data and metadata; which is one of the challenges in genomic data exchange between heterogeneous systems Sansone et al., 2008. One of the most common tools is ISA-TAB which is stand for Investigation-Study-Assay (ISA) tab-delimited (TAB) (http://www.isa-tools.org/). According to Jameson et al., 2011, ISA-TAB is initially developed as a standard in typical tabular format to describe data from diverse forms of omics experiments. This standard is mainly created to allow 
easy data exchange of metadata in the future between public resources. These data particularly is "omics-based" experiments such as genomics, proteomics etc. In ISA-TAB, data are organized in tab delimited file which can be viewed as textual table representation using ISA-TAB web viewer for ISA-Tab files that found in (https://github.com/ISA-tools/ISATab-Viewer).

\subsection{Motivation}

Many organisms have been completely sequenced today. Consequently, there is high demand for visualization tools for the data collected about a whole genome such as genes, proteins, annotations, and expression data. Those tools are essential to understand what a gene and gene product do. Since the genes role can be noticed by watching some patterns, visualization in tabular (spreadsheet) format is one of the important methods to organize and identify these patterns. There are many special tools that created for visualizing the gene structure in non-tabular format such as FancyGene Rambaldi and Ciccarelli, 2009 and GECA Fawal et al., 2012. However, they have limited visualizing options. For example in FancyGene there is rich annotation information for the gene model but it only shows a single gene. In contrast, GECA displays many genes but the features are limited. There is a need for visual representation for all the genes in the genome to be in a single place.

In our research, we put the annotated information about the gene and its encoded protein in a single place, and gather them in tabulate view to allow the user to explore patterns much easier. In our system, the protein feature that are associated to the gene is presented in single row with the gene that belongs to while this feature is highlighted on the top of gene models on FeatureStack Frech et al., 2012

\subsection{Research Challenges}

While data visualization is increasingly important, its design is challenging since it involves considering many aspects related to the problem domain and the end-users. According to Krzywinski, 2013], the human ability to read visually is extremely sophisticated, so applying some principles will be required to have useful and usable visual system. Some of the important principles that considered in this thesis are stated in Chapter 2 Section 2.5.

In addition, usability is one of the big challenges in visualization since many powerful systems are available but the big question is: are they useable or not. Currently, researchers have started to act seriously and pay more attention to usability analysis. In Wang Baldonado et al., 2000 paper, there are some guidelines that should be considered in designing usable visualization systems, which will be discussed in Chapter 2 Section 2.5.2. Since the targeted end-users are the most important thing while designing our system, we try to follow the common visual designs as there are metaphors familiar to users. 


\subsection{Research Contributions}

The general goal of the thesis is to develop a web-based system that provides the visualization of genome annotation data in single spreadsheet. In order to achieve this, the contributions of this thesis are the following:

- Analyze different annotation files about different fungal genomes with different file formats from the AspGD database (www.aspgd.org) and InterPro (http://www.ebi.ac.uk/interp ro). Then, extract the key features that required to display the following: gene model, protein domains, genes location in the chromosome and GO Slims availability in the genome.

- Implement a new approach of presenting genome analysis data, which is HTML5 visual spreadsheet to express genome annotation data. The system can be accessed from any device with a web browser.

- In the CGene system, we lined up the gene structure with protein domain architecture in a single row of the spreadsheet. Each genome, that may have more than 10000 genes, will be presented in a single spreadsheet which allows the scientist to explore genomic patterns easily. The visual image of a gene model for each genes in the genome is given in Scalable Vector Graphics (SVG) format. Any single image can be zoomed in/out without loosing image details and can be downloaded as SVG file. In addition, the protein features are presented in the row with the gene. The protein domain architecture is also presented in SVG format.

- In the CGenome system, we lined up different genomes in which each row in the spreadsheet represents a single genome. In each row of the spreadsheet we display the following in SVG format: Gene location, Go Slims bitmap and Go Slims histogram.

\subsection{Organization of Thesis}

The thesis document is organized the follows:

- In Chapter 2, we present some background basics that are needed to ease the understanding of the developed system. The background includes some biology, bioinformatic basics and principles for visualization. This chapter also includes some of the examples about the available visualization systems that used to display genomic context data.

- In Chapter 3, a detailed description of $\boldsymbol{C G}$ ene and $\boldsymbol{C G}$ enome systems, pronounced See-Gene and See-Genome respectively, is presented. The details include: system requirements, system analysis, system construction and system user interface.

- Chapter 4 has the conclusion. This chapter summarizes the contributions, states limitations, and makes some suggestions for future work. 


\section{Chapter 2}

\section{Background and Basic Concepts}

\subsection{Basic Concepts of Biology}

\subsubsection{Cell, Nucleic Acids and Amino Acids}

A cell is the basic building unit of creatures and living species. The human body is made of trillions of cells which come in many different varieties with many different functions. Each of the cells has the complete human genome on its nucleus, which composes of 23 pairs of chromosome. Each chromosome have a collection of DNA(Deoxyribonucleic acid) molecules that encode the genetic code which used in building and functioning of the human being and the different organisms Majoros, 2007].

While DNA is found in the nucleus of eukaryotic cells, prokaryotic cells lack a membrane-bound nucleus. As shown in Figure 3, each chromosome has a collection of DNA double helix stranded molecule that determined in 1953 by Watson and Crick. This determination opened the way to understand most important biological process more deeply. DNA composes of sugar and phosphate backbone and four different bases $\{$ ATCG $\}$ : Adenine (A), Thymine (T), Cytosine (C) and Guanine $(\mathrm{G})$. The pairing between these bases called Watson-Crick complementarity in which the bases are represented in the following rule: $C \Leftrightarrow G, A \Leftrightarrow T$ ( $A \Leftrightarrow U$ in case of Ribonucleic acid (RNA)) Watson et al., 1953.

Small parts of DNA strand are called genes. Genes control a hereditary characteristic and determine the physiology of the organism. The strand that contains the gene is called the template (or sense strand) for that gene while the complementary strand is referred as the reverse strand (or antisense strand). 


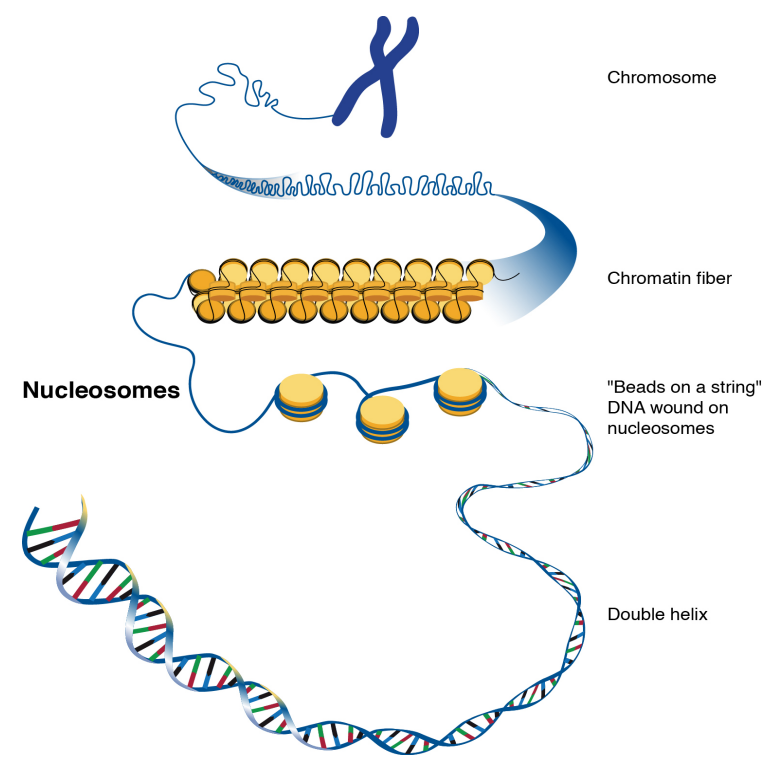

Figure 3: Illustration of chromosome that have DNA double helix stranded molecule.(Credit: Darryl Leja, The National Human Genome Research Institute (NHGRI))

A protein is a large biological molecule, which its primary structure is the sequence of one or more long amino-acid residues. A protein sequence is represented as a chain of 20 English alphabet letters $\{\mathrm{A}, \mathrm{C}, \mathrm{D}, \mathrm{E}, \mathrm{F}, \mathrm{G}, \mathrm{H}, \mathrm{I}, \mathrm{K}, \mathrm{L}, \mathrm{M}, \mathrm{N}, \mathrm{P}, \mathrm{Q}, \mathrm{R}, \mathrm{S}, \mathrm{T}, \mathrm{V}, \mathrm{W}, \mathrm{Y}\}$ where each letter represents an amino acid as shown in Table 1 .

\begin{tabular}{|c|c|c|c|}
\hline Amino Acid & Alphapet & Amino Acid & Alphapet \\
\hline Alanine & A & Methionine & M \\
\hline Cysteine & C & Asparagine & N \\
\hline Aspartic acid & D & Proline & P \\
\hline Glutamic acid & E & Glutamine & Q \\
\hline Phenylalanine & F & Arginine & R \\
\hline Glycine & G & Serine & S \\
\hline Histidine & H & Threonine & T \\
\hline Isoleucine & I & Valine & V \\
\hline Lysine & K & Tryptophan & W \\
\hline Leucine & L & Tyrosine & Y \\
\hline
\end{tabular}

Table 1: Proteins' 20 amino acids

\subsubsection{Central Dogma of Molecular Biology}

The central dogma of molecular biology deals with the detailed residue-by-residue transfer of sequential information. It states that such information cannot be transferred from protein to either protein or nucleic acid. Crick et al., 1970]

In 1958, the central dogma of molecular biology was first stated by Francis Crick, and then it was 
re-stated and published in a Nature paper 1970 [Crick et al., 1970. It is a framework for describing the genetic information flow from DNA to messenger RNA (mRNA) molecules then to proteins. The transfer from DNA to mRNA is done through a process called transcription, in which a fragment of DNA sequence is copied into RNA by the enzyme RNA polymerase. Then protein is synthesized by using the information in mRNA as a template through an irreversible process called translation as illustrated in Figure 4 as a summary of central dogma of molecular biology.

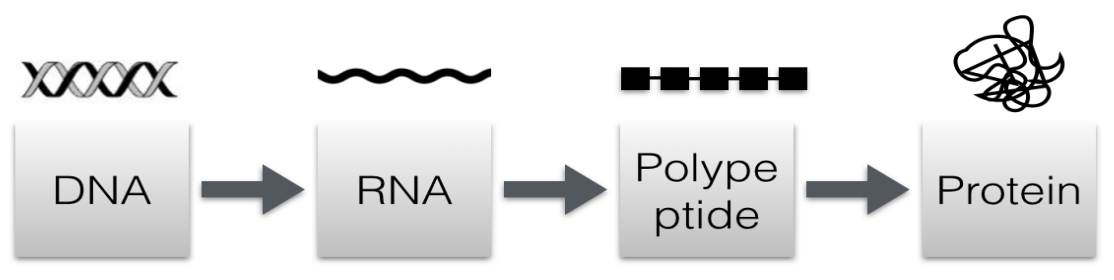

Figure 4: A summary of Central Dogma of Molecular Biology

During the transcription process, the enzyme $R N A$ polymerase is used to copy a particular segment of DNA into RNA. Then, to produce messenger RNA (mRNA) that will act lately as template for protein synthesis, the RNA nucleotides are joined by strong phosphodiester bonds. Before the translation process, the transcript (or pre- $m R N A$ ) is spliced by large and complex molecular machine called spliceosome. Its job is to remove portions of RNA known as introns from the sequence and keeps the exons as shown in Figure 5.

In translation, $m R N A$ is decoded by a ribosome complex which moves from codon to codon along the mRNA to produce a chain of amino acids. After terminating translation, a complete polypeptide is released from the ribosome which will later fold into an active protein Majoros, 2007.

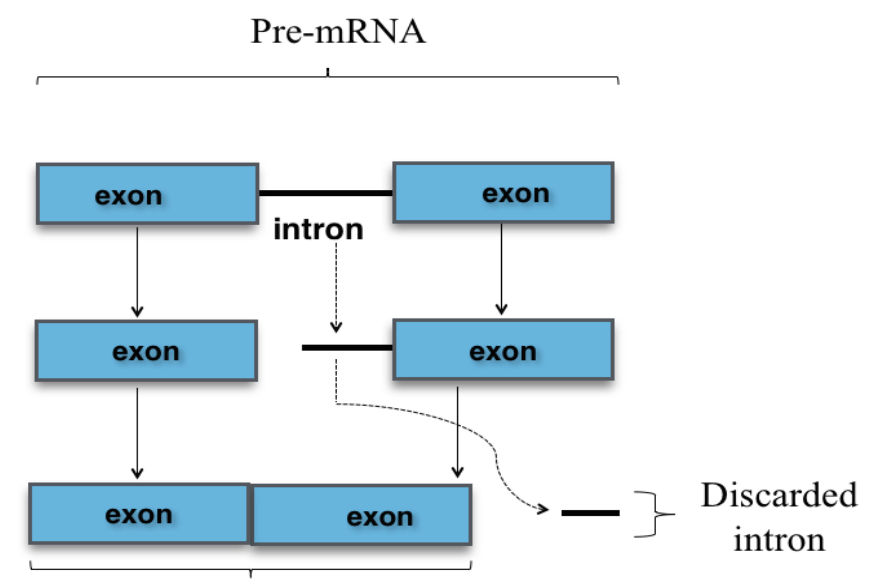

Mature RNA

Figure 5: Removal of introns during the splicing process to produce a mature mRNA in eukaryotic gene 


\subsection{Gene and Gene Model}

\subsubsection{Genes, Exons, Introns and Coding Segments}

As mentioned before in translation process at Section 2.1.2, the continuous sequence of bases in RNA transform later to protein. However, not all these sequences convert to protein. Specifically, this means not all exons that found in the gene are translated to protein, only the specific part called the coding region (or coding segment $C D S$ ) that encode the actual recipe for gene's product. The coding segment usually starts with a start codon (or translation start site) and terminates with a stop codon (or translation stop site). Lying in between, there are exons that only translated to protein which located apart from the five prime region and three prime region of the start and stop codons.

\subsubsection{Gene Model}

A gene model usually is drawn to reflect the location of introns and exons relative to the genomic sequence Majoros, 2007]. In addition, it shows where the RNA polymerase started transcription and the location of the starts and stops of translation as seen in Figure 6. In this figure, a general eukaryotic gene model and $A$ acidus $C B S 106.47$ fungi gene model are illustrated. Both gene models in the figure have multiple exons and introns.

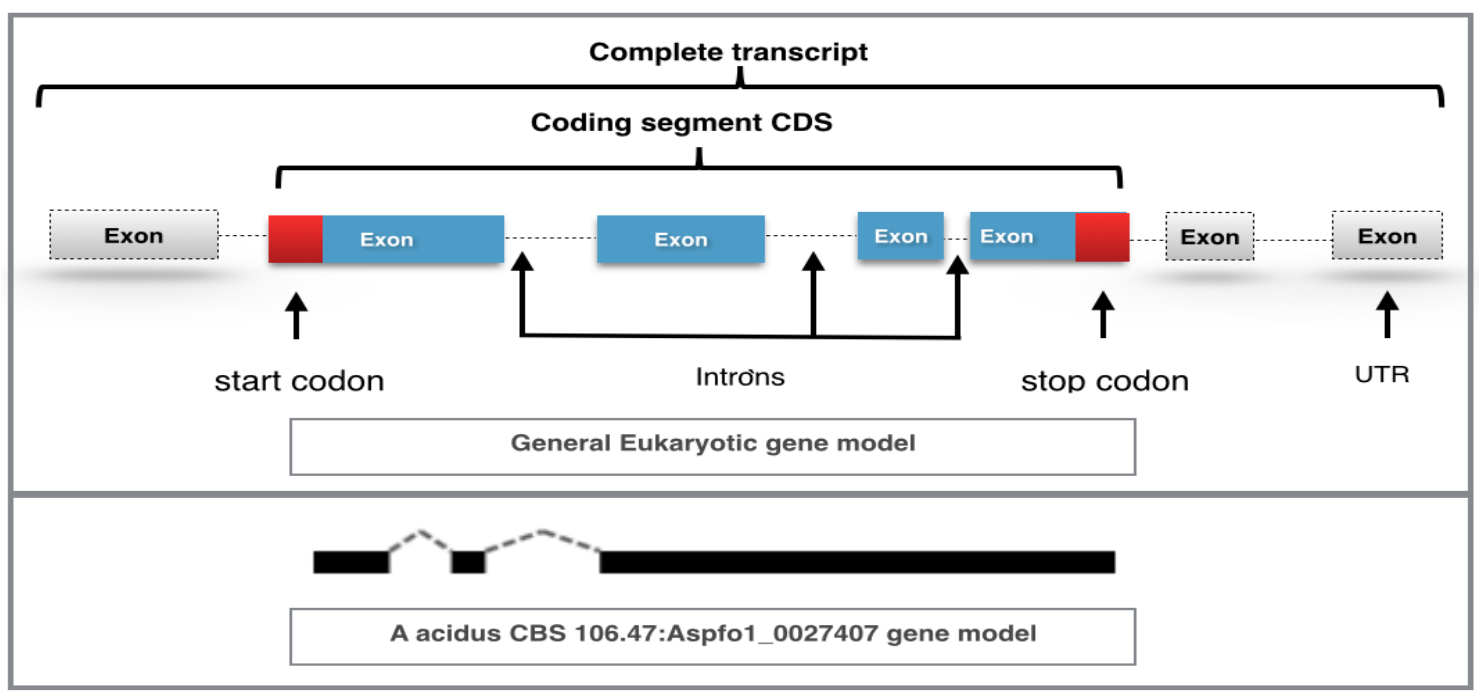

Figure 6: Gene Model in eukaryotic gene. It illustrates the coding segment (CDS) that contains the exons connected by introns. The grey rectangles are the untranslated regions (UTR). The gene model below is for one of the genes in A acidus CBS 106.47 fungi.

It is worth to mention that in eukaryotic gene, some parts of the original gene, called introns, are removed during the splicing process in order to complete the translating process. In case of prokaryotic gene, it is simpler since it does not have introns. Each intron begins with donor splice site and end with acceptor splice site. Codons work as signals in DNA sequence and they have 
specific codes. ATG is the code for start codon while TAG, TGA, or TAA are the codes for stop codons. GT and AG are the codes for splice donor site and acceptor donor site respectively. However, the identification for these codons in DNA sequence is not distinct which means not every presence of ATG is start codon and not each AG is acceptor donor site Majoros, 2007.

\subsection{Gene Annotation and Gene Ontology}

\subsubsection{Gene Annotation}

Over the years, the amount of the genome sequencing data has expanded significantly. As we see in Figure 7, the number of Genome Sequencing Projects in Genome Online Database (GOLD) has been increased generally from 2007 to 2014. The figure also shows the growth of sequence databases and annotation since 1982. Sequence discovery helps for identifying new genes, observing chromosome organization and structure, and for performing comparative genomics. These data may lead to advance in fields such as medicine, agriculture etc.

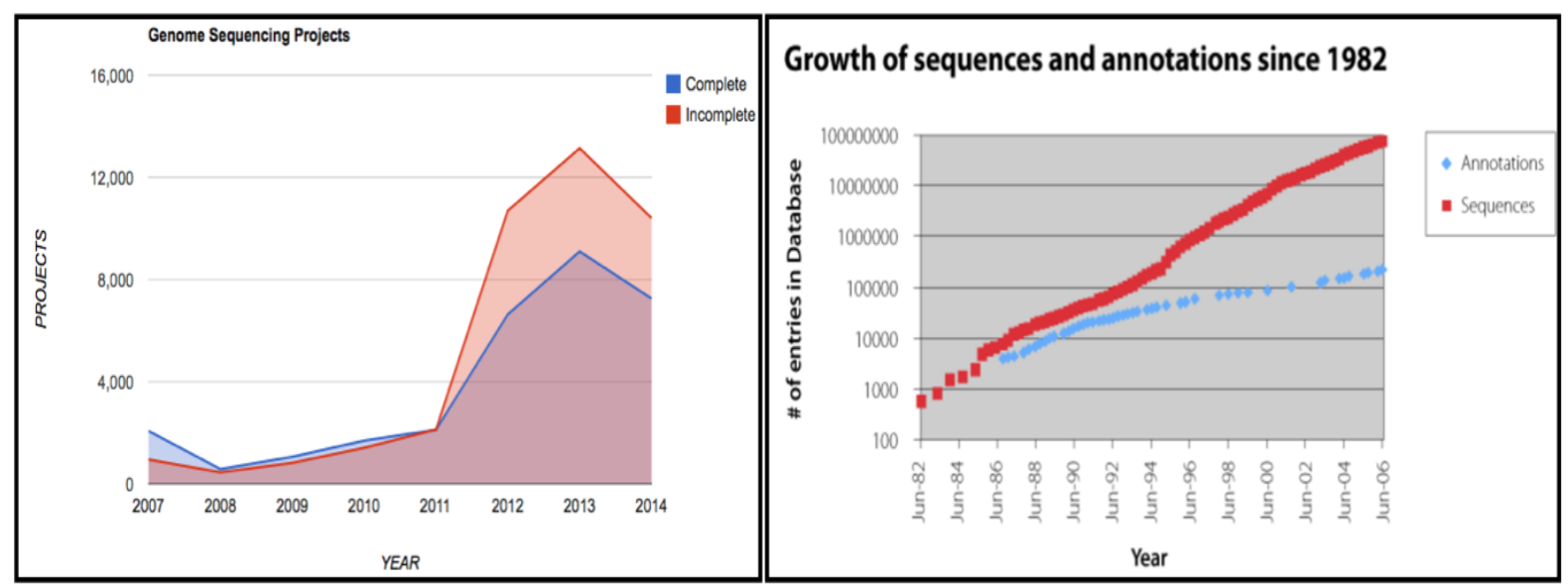

Figure 7: The graph in the left illustrates the increased amount of Genome Sequencing Projects in Genome Online Database (GOLD) from year 2007 to 2014. The graph in the right represents the growth of sequence databases and annotation since 1982. The numbers are taken from of the databases Genbank(NCBI) and Swissprot(EBI). Credit: (Genome Online Database (GOLD) and (Folker Meyer, Argonne National Laboratory).

As much as this data are discovered, it does not become useful for biologist until its components are identified and analyzed Rouzé et al., 1999. The process of attaching identities and functions to sequences is called genome annotation. Genome annotation is sub-field of genome analysis, which deals with computational processes in genome sequences.

The main purpose of genome annotation is to find the key features of the genome, particularly the genes and their products Stein, 2001. After the sequences are discovered, some steps are done on the sequences to achieve the extracted results. This is done by following schematic data flow, as shown 
in Figure 8 from Koonin EV, 2003, to produces these key features. The steps are starting with identifying genome elements that encodes genes, then predicting some functional elements. These steps are integrated with statistical gene prediction, structural features prediction, and database searching. The database searching for sequence similarity can be done in general databases such as NCBI non-redundant database or specialized databases such as Pfam and SMART.

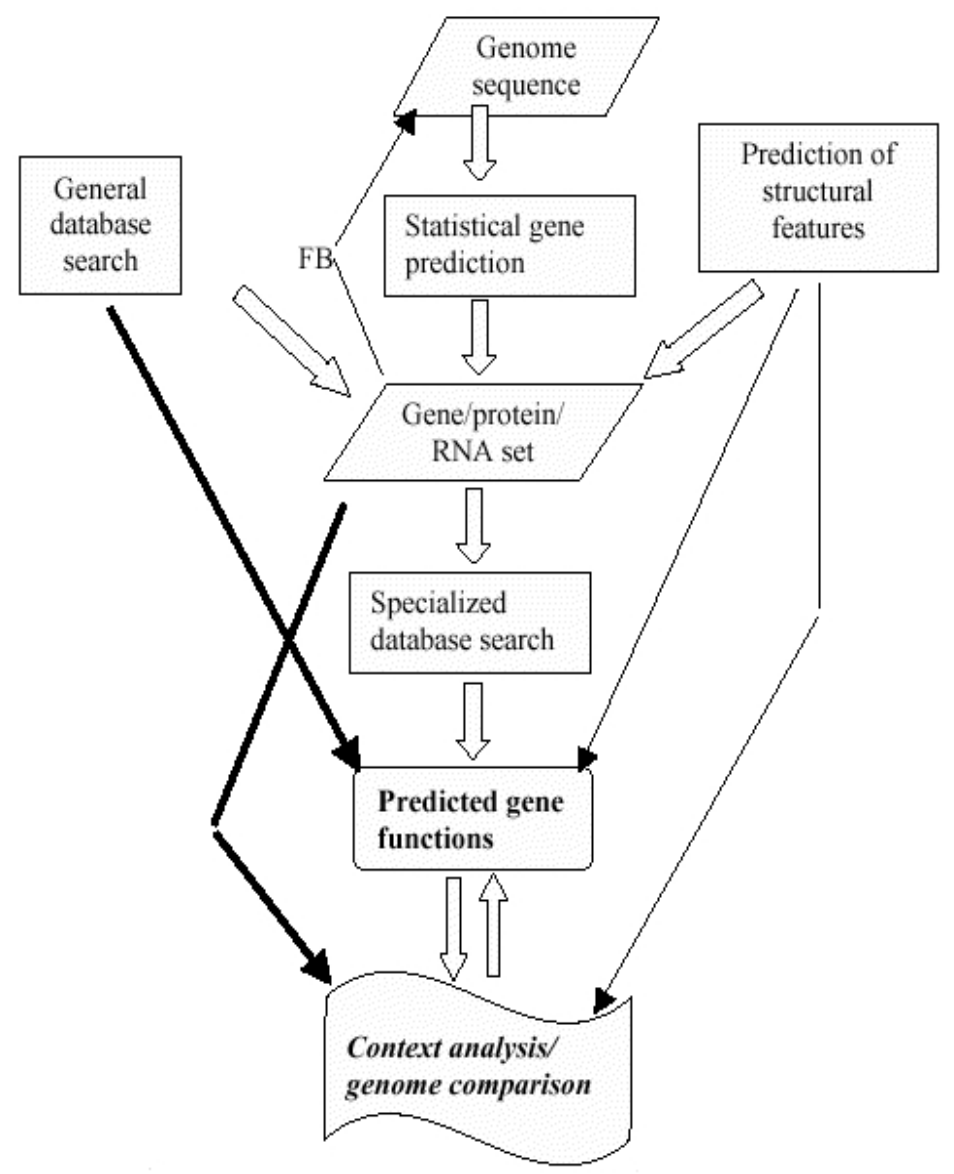

Figure 8: Generalized flow chart illustrates Genome Annotation procedure. The steps are integrated with statistical gene prediction, structural features prediction, and database searching to produces genome key features. Credit: ( Koonin EV, 2003 paper. For further reading about the steps in the chart pleas visit: (http://www.ncbi.nlm.nih.gov/books/NBK20253/))

Many computational tools have been developed in order to help in generating genome annotation. One of the most basic and common tools is Basic Local Alignment Search Tool (BLAST) http: //www.ncbi.nlm.nih.gov/BLAST/), which allows the user to query protein or nucleotide sequence and find its similarities against a large number of sequences. The genome is annotated based on that similarity. However, doing the whole process in BLAST then waiting the results is time consuming especially if the number of sequences is huge Koonin EV, 2003. Consequently, it is essential to use some level of automation in genome annotation by using some softwares that do the query 
process then organize the results. After that, the expert is responsible to assign gene functions manually or automatically such as GeneQuiz (http://www.sander.ebi.ac.uk/genequiz/) project, which is general public automatic system for genome analysis. In GeneQuiz, results evaluation and function annotation initiation are done automatically by an expert system after similarity search is finished Koonin EV, 2003.

\subsubsection{Gene Ontology}

As we discussed in the previous section, many genes are identified. Consequently, we need to assign names and functions for them. It is common in biology to have more than one word to describe the same phenomenon such as citric acid cycle, which also called tricarboxylic acid cycle (TCA cycle) and the Krebs cycle. The need for uniform vocabulary and naming structure for genes, its products and their relationships became essential. For this reason, Gene Ontology (GO) project has been developed as cooperative effort between three databases: FlyBase (http://flybase.org), the Mouse Genome Database (MGD) (http://www.informatics.jax.org), and the Saccharomyces Genome Database (SGD) (http://www.yeastgenome.org) GeneOntology, 2014.

The main role of this GO consortium (http://www.geneontology.org) is to create a systematic language that has consistent vocabulary and description for genes and its products across different databases Gene Ontology Consortium and others, 2008. Each GO term is given a name and unique number that consists of seven digits in the form of GO:nnnnnnn such as GO:0005102 for receptor

\section{binding.}

The GO terms are divided into three aspects based on the biological domains, which are Molecular Function, Biological Process, and Cellular Component. Molecular Function explains activities that happen at the molecular level such as catalysis or binding activities. Biological Process explains the higher-level activity that the molecular function provides such as DNA metabolic process. Cellular Component describes the cell and its environment such as chromosome, ribosome, or cytoplasm.

GO terms can be assigned to a UniProtKB entry in electronic or manual way. Each term is associated with a specific reference to describe what type of analysis done to identify the association between the GO term and the gene product. This reference called evidence code which each annotation must has to indicate how the annotation to a specific term is supported GeneOntology, 2014. For example, IEA is a code for Inferred from Electronic Annotation which is used when annotation transferred from database or derived from computation.

These terms are not organized in a tree structure, but instead they organized in a directed acyclic graph, in which one term can have more than one parent term. In Figure 9, we see an example of regulates and part_of relationships between Molecular Function and Biological Process Gene Ontology Consortium and others, 2010.

GO terms are increasing and the relationship between the three branches has been expanded too. In Table 2, a summary of the GO content in the years 2007 and 2010 is presented. This table shows that terms are increased in biological process from 13916 to 17069 terms, in molecular function from 7878 to 8637 terms and in cellular component from 2007 to 2432 terms.

There are browsers for GO terms such as QuickGO, which is a open-source web-based browser 
for Gene Ontology terms and annotations searching. This browser is developed by the UniProt-Gene Ontology Annotation (UniProt-GOA) project (http://www.ebi.ac.uk/QuickGO/). In QuickGO, as shown in Figure 10, a GO term can be viewed alongside with its definition, synonyms, child terms, etc.

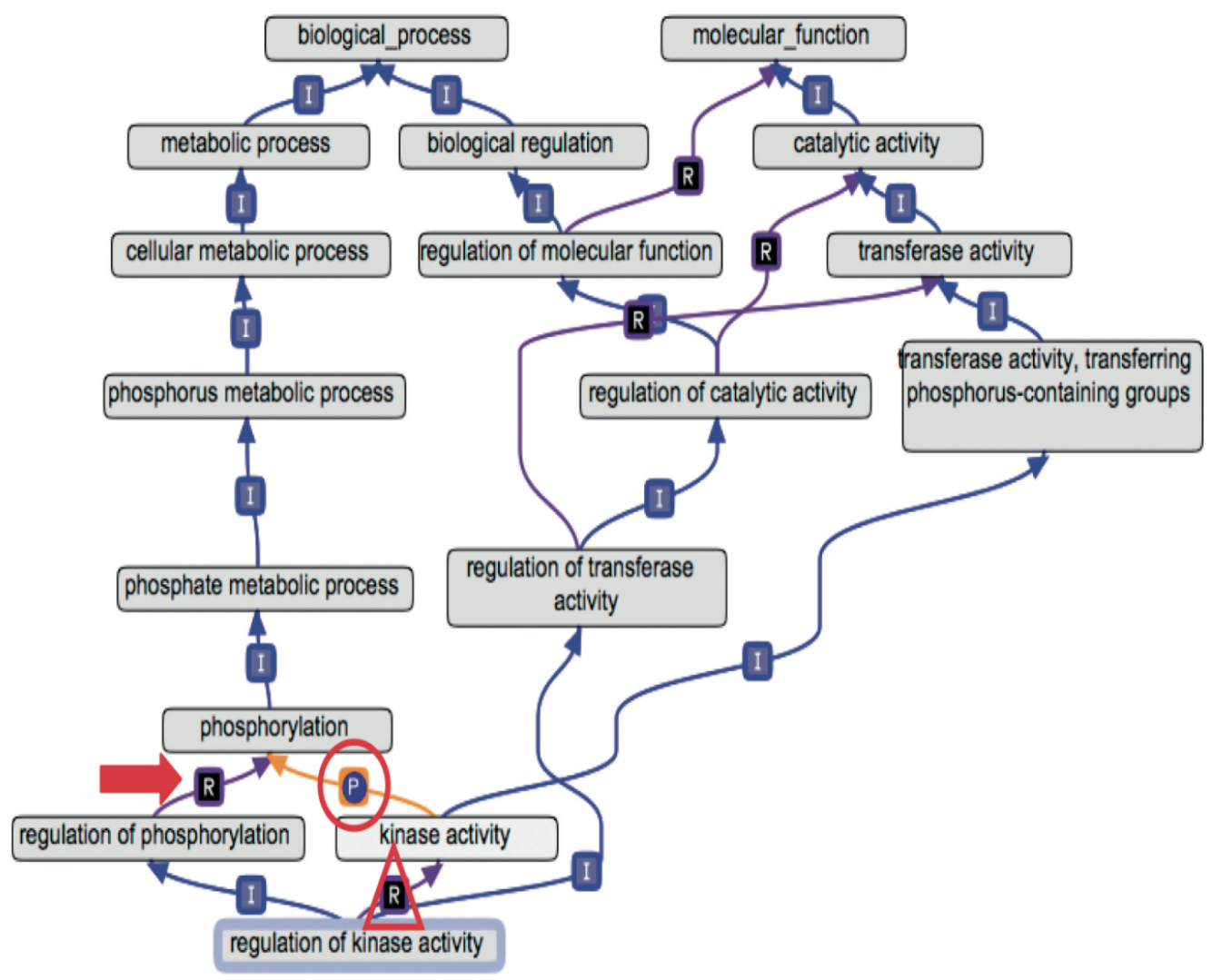

Figure 9: Graph shows regulates and part_of relationships between Molecular Function and Biological Process. Triangle indicates a regulates relationship and circle indicates part_of relationship between them. Credit: ( Gene Ontology Consortium and others, 2010]) 
Table 2: The status of Gene Ontology as September 2007 and September 2009, which shows increase in Go terms between 2007 and 2009. It shows that terms are increased in biological process from 13916 to 17069 terms, in molecular function from 7878 to 8637 terms and in cellular component from 2007 to 2432 terms. Sources: Gene Ontology Consortium and others, 2008 and Gene Ontology Consortium and others, 2010.

\begin{tabular}{|c|c|c|}
\hline & \multicolumn{2}{|c|}{ Gene Ontology status } \\
\hline & Gene Ontology status in 2007 & Gene Ontology status in 2009 \\
\hline Biological process terms & 13916 & 17069 \\
\hline Molecular function terms & 7878 & 8637 \\
\hline Cellular component terms & 2007 & 2432 \\
\hline Sequence ontology terms & 1305 & 1603 \\
\hline Annotation datasets ${ }^{\mathrm{a}}$ & 35 & 52 \\
\hline \multicolumn{3}{|l|}{ Annotated gene products } \\
\hline Total & 3347495 & 44545253 \\
\hline Electronic $^{\mathrm{b}}$ & 3128309 & 43655159 \\
\hline Manual & 219186 & 890094 \\
\hline
\end{tabular}

Note: Gene Ontology Consortium and others, 2010] ${ }^{\text {a }}$ : Most datasets represent single species.

b: Annotations using the IEA (inferred from electronic annotation) evidence code.

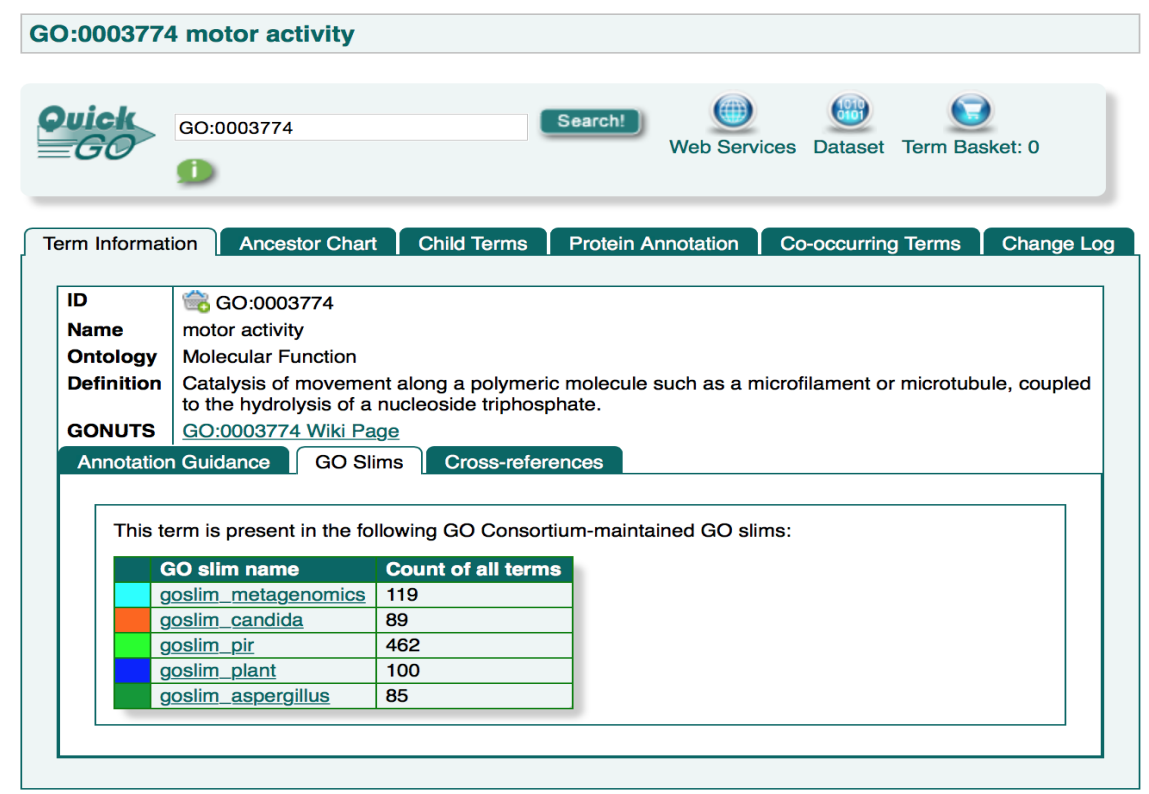

Figure 10: Gene Ontology terms and annotations from Quick $G O$ web browser, where GO term can be viewed alongside with its definition, synonyms, child terms, etc. 


\subsubsection{Go Slims}

It is difficult to handle all terms in the gene ontology, especially for biologists who focus in specific species or who do not need a lot of details about the terms. Consequently, the need for cut-down versions of the GO ontologies became necessary. Go Slims terms are a sub-list of whole GO terms that give general outline of the ontology content without many details. GO Slims are useful for having a general classification of gene products for a genome, microarray, or complementary DNA (cDNA) collection GeneOntology, 2014. There are generic GO slims, which are suitable for general purposes, since they are not species-specific. In the other hand, there are species-specific slims that are developed for specific organisms or groups of organisms. For example, there is Yeast slim which developed by Saccharomyces Genome Database (http://www.yeastgenome.org) and Candida albicans which developed by Candida Genome Database (http://www.candidagenome.or g). In addition, biologists can create their own slims by contacting Gene Ontology Consortium GO helpdesk. All these subsets can be downloaded from Gene Ontology Consortium website (http: //geneontology.org/page/download-ontology).

Below in the box is an example for GO term named motor activity from GeneOntology, 2014 for Aspergillus slim that developed by Aspergillus Genome Data (http://www.aspgd.org), which our research group is focus on, and is used in this research in CGenome visual spreadsheet.

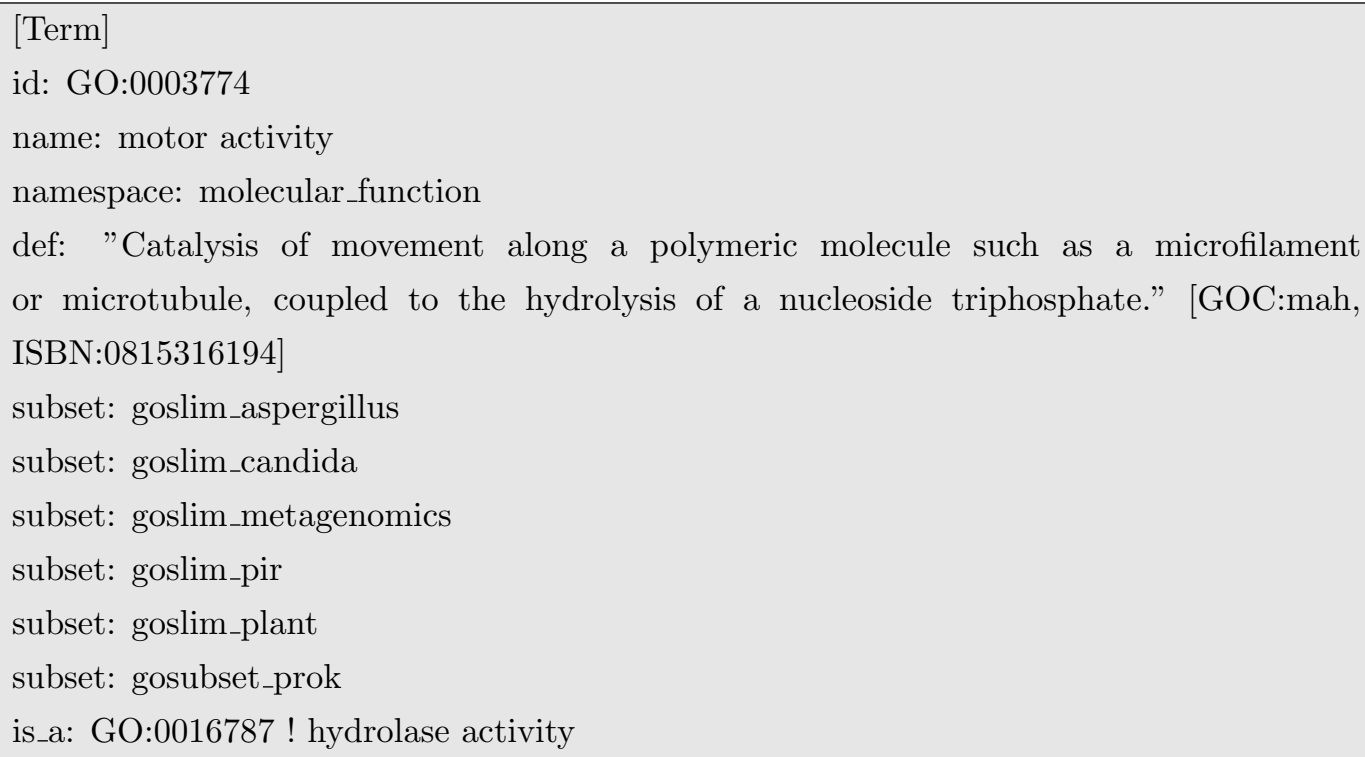

\subsection{Protein Structure, Protein Domain and its Architecture}

\subsubsection{Protein Structures}

Generally, protein structure is divided into three or four levels structure based on the references that categorized the protein structure. According to Rangwala and Karypis, 2010, protein structure is divided into four levels which are primary structure, secondary structure, tertiary structure, and 
quaternary structure. Primary structure is the main level structure of the protein which is the Amino Acids Sequence. There are mainly 20 different amino acids that form the building protein sequence blocks which stated previously in Section 2.1.1.Table 1 These amino acids is linked by peptide bonds to form the sequence chain. The secondary structure composes the second level of protein structure. It consists of some regions, which form themselves independently from the rest of the protein into repeated patterns that are occurring in the protein. The most common forms of the secondary structure are $\alpha$-helices which has a coil-like structure, and $\beta$-sheets that contains parallel strands of residues. These regular structures can have critical role in biological function. Tertiary structure is the global 3D shape that represents the protein in three dimensional crystal coordinate. This shape contains of a variety arranged secondary structure elements that bonds together. Quaternary structure represents the interaction between various polypeptide chains which happens because of the "non-covalent interactions between the atoms of the different chains" Rangwala and Karypis, 2010.

\subsubsection{Protein Domains}

The word domain denotes a certain region in the protein such as domain family. According to Morrison, 2013, protein domains are high-level representation for protein parts. These domains can fold independently from the rest of the protein sequence into a tertiary (three-dimensional) structure. A protein sequence can have many domains, and most of them have a specific function or participate with other domains in particular function on their protein.

An example of protein domain is BAR (Bin-Amphiphysin-Rvs) domains, which shaped as banana shape and binds to membrane through its concave face, are extremely conserved protein dimerization domains. These domains occur in several proteins involved in membrane dynamics in a cell Peter et al., 2004. A single protein domain can be structured into a three-dimensional structure. To represent protein sequence that has multiple or single domain, we can use protein domain architecture as represented in Figure 11. In this figure, a 3D crystal structure of Endophilin $\boldsymbol{B A \boldsymbol { R }}$ domain and the domain architecture representation for the same domain are illustrated.

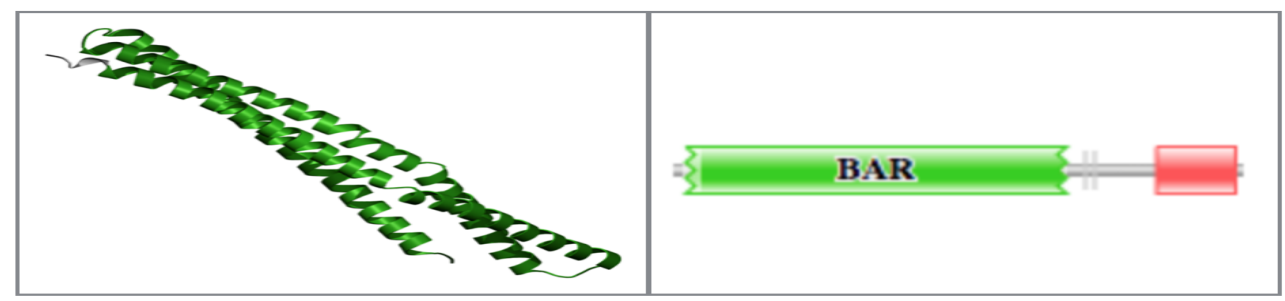

Figure 11: In the left image is the 3D crystal structure of Endophilin $\boldsymbol{B} \boldsymbol{A} \boldsymbol{R}$ domain, while the right image is the domain architecture representation for BAR Domain in Endophilin-B1 from

Pfam DB. Credit: both images are taken from Pfam DB 


\subsubsection{Protein Domain Databases}

There are many domain databases (DBs) that store protein annotation resources. These DBs differ on the way that they are constructed by Morrison, 2013. Below is a list of the most common protein DBs each associated with URL for further details.

- SCOP: it is stand for Structural Classification of Proteins. It uses the information that gathered and experimentally determined from protein 3D structure. For more details: http://scop.mrc-lmb.cam.ac.uk/scop/.

- CATH: it is a classification DB of protein structures which are downloaded from the Protein Data Bank. For more details: http://www. cathdb.info.

- SUPERFAMILY: it is a structural and functional annotation DB for all proteins and genomes. It uses SCOP domains and assigns them to protein sequences by matching them using Hidden Markov Models (HMMs). For more details: http://supfam.cs.bris.ac.uk/S UPERFAMILY/

- Gene3D: it is constructed in a way similar to SUPERFAMILY, but using CATH domains instead of SCOP domains. For more details: http://gene3d.biochem.ucl.ac.uk/Gen e3D/.

- Pfam: it is a large DB that has many protein families which represented by multiple sequence alignments (MSA) and hidden Markov models (HMMs). For more details: http://pfam .xfam.org.

- NCBI CDD: it is stand for Conserved Domain Database that created by the National Center for Biotechnology Information. It consists of a group of sequence alignments and profiles that represent conserved protein domains in molecular evolution. For more details: http: //www.ncbi.nlm.nih.gov/cdd/.

- SMART: it is stand for Simple Modular Architecture Research Tool. It is manually curated DBs that mainly focus on signaling and extracellular domains. For more details: http: //smart.embl-heidelberg.de

- INTERPRO: it is meta-database that combined its data from several DBs such as Pfam. For more details: http://www.ebi.ac.uk/interpro/.

- UniPort: it is stand for Universal Protein Resource, which has collection of protein sequence and functional information. For more details: http://www.uniprot.org.

- ProDom: it is automatically created DB that gathered from Uniport For more details: http: //prodom.prabi.fr/prodom/current/html/form.php.

- ADDA: it is stand for Automatic Domain Decomposition Algorithm. Its concept is similar to ProDom since its data are gathered and clustered form several Dbs. For more details: url http://ekhidna.biocenter.helsinki.fi/sqgraph. 


\subsubsection{Protein Domains Architecture}

Domain architecture, or domain arrangement, refers to the domains and their arrangements in individual protein. The direction of these domains goes along the amino acid chain from $\mathrm{N}$ - to $\mathrm{C}$ terminal [Morrison, 2013]. The design of the shape and the colour of each domain differ depending on the system that built the architecture as seen in Figure 12. In this figure, protein domain architecture from SMART, ProDom, InterPro and Pfam is illustrated. Most of the domains in those systems use the rectangular shape with or without rounded or jagged edges. Generally, the rectangular shape is the most common shape that used to represent the domain, however some systems use different shapes for different domain families such as SMART system DB. Other systems such as Pfam use rectangle for the domain with different colour for different domain families. The colour is also varying between the systems and some of them use patterns such as ProDom. In Appendix B. Table 12, there is a descriptive catalog for Pfam domain architecture graphics design. For more examples about protein domain architecture in different systems see Appendix B.

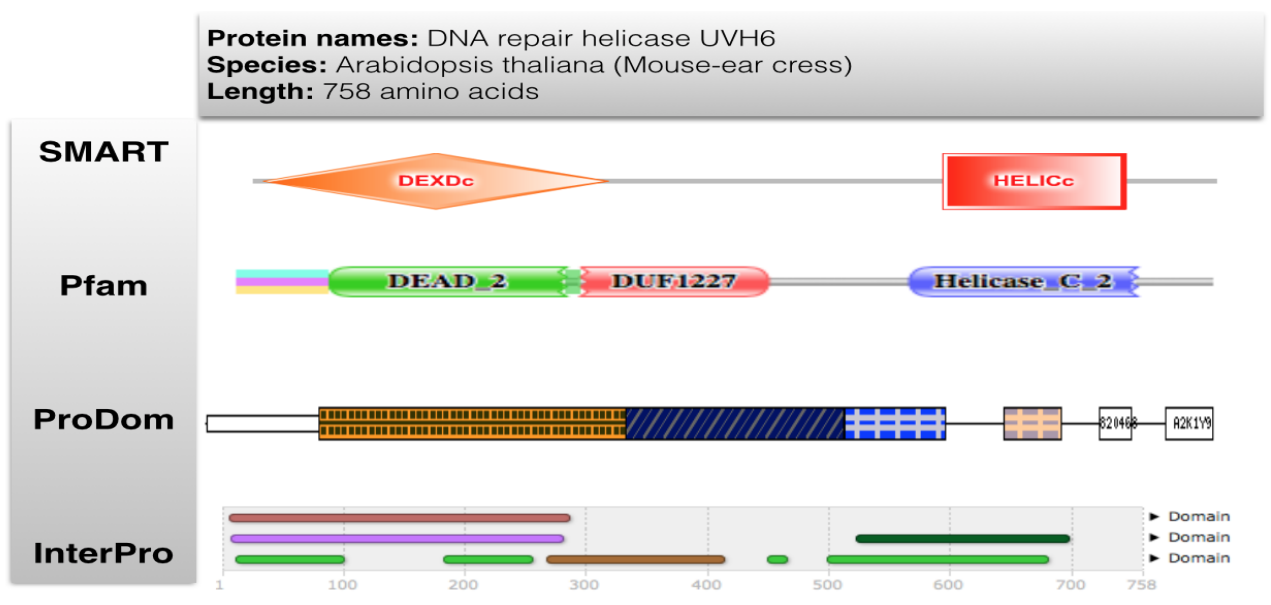

Figure 12: Domain architecture representation for DNA repair helicase $\boldsymbol{U} \boldsymbol{V H} \boldsymbol{6}$ protein which belong to Arabidopsis thaliana (Mouse-ear cress) Organism. It is gathered from four different databases: SMART, ProDom, InterPro and Pfam. The rectangular shape is the shape that used to represent the domains in ProDom, InterPro and Pfam while SMART uses different shapes. The color is varying between the systems while ProDom uses colors and patterns for domain architecture displaying.

\subsection{Principles of Biological Data Visualization}

In some situations the ability to display data in visual format is difficult especially with a massive data such as biological datasets. Consequently, the computer-based system is required to save the human efforts. For computer-based visualization to be effective tool, some visualization principles should be considered during the visualization design such as visual representation understanding and multiple views system building principles. In the next two Sections 2.5.1 and 2.5.2 these principles 
will be discussed.

\subsubsection{Visual Representation Understanding}

In order to understand the visual representation, we need to analyze three important questions first, which are: what is the data type that we are willing to show, why are users looking at this data, and how to show the data Munzner, 2014. To answer these questions clearly, we need to divide the analysis to four levels which are domain situation, abstraction, idiom and algorithm. These three questions are found inside the four levels as seen in Figure 13 Munzner, 2014.

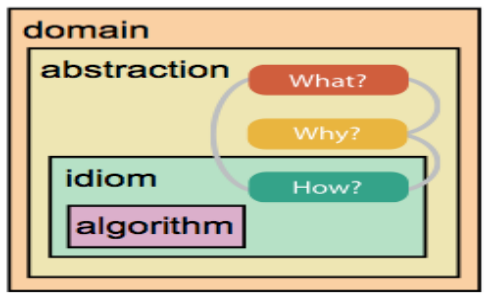

Figure 13: Illustration of A Multi-Level Typology of Abstract Visualization Tasks. These levels are needed to answer three important question in visualization, which are: what is the data type that we are willing to show, why are users looking at this data, and how to show the data. ( Credit: Brehmer and Munzner, 2013])

Each system has its own targeted users, and a visualization tool has to be more than just graphical demonstration of information when its users are scientific researchers Wong, 2012. The objectives and researchers' expectations need to be considered while designing the system. After knowing the domain that the system is targeting, there is a need to think deeply about what does the data that need to be displayed means in the real world and what is the type of this data Munzner, 2014.

The semantic, which care about what does the data mean, is very crucial in visualization in order to see how to group or organize this data. In addition to the semantic, the structural or mathematical data type is important to perform visualization analysis. According to Munzner, 2014, there are different categories for the dataset type which can be tables, networks, fields and geometries. Table data type where the data is represented as a combination of rows and columns and their intersections is a value in a single cell. Network where there is a relationship between two or more items. The data is represented as nodes (or vertex) and multiple links (or edges) connecting these nodes based on the relationship between them. In field dataset type there are values in cells, but these cells have measurements from continues domain where a new measurement can be taken between two available cells. These measurements could be pressure, temperature, speed, density etc. For example, if a scan of human body is made and spread through a volume of 3D space. The density of the tissue at the sample could be taken apart for a coarser grid, or closer with a higher resolution grid of cells Munzner, 2014. In table and network data types the value is discrete, and showing values in between them is not a meaningful concept. The geometry dataset type has information about "the shape of items with explicit spatial positions" Munzner, 2014. These 
items could be points, one-dimensional lines, 2D surfaces, or 3D volumes. Each one of these dataset types may have a combination of types of data which are attributes, links, items, positions and grid cells. These data types describe the values and their relationships and positions. A summary of these dataset types and their types is illustrated in Figure 14 To encode the data, we need to categorize the data such by their colour, shape, specific pattern or all of them. We also need to group them even by one or combination of connection, similarity, or positions. Then the data need to be ordered by ordinal or quantitative order by saying the data size is small or its measure is 10 inches. At data abstracting stage, it is essential to translate the targeted data from a specific domain to visualization vocabulary in a process called the visual encoding of data in order to display it later on. After analyzing the data, an efficient computation algorithm has to be applied based on the system objectives. In the next Section 2.5 .2 , there will be a suggested principles of how to build a successful visualization system in biology.

\section{Dataset Types}

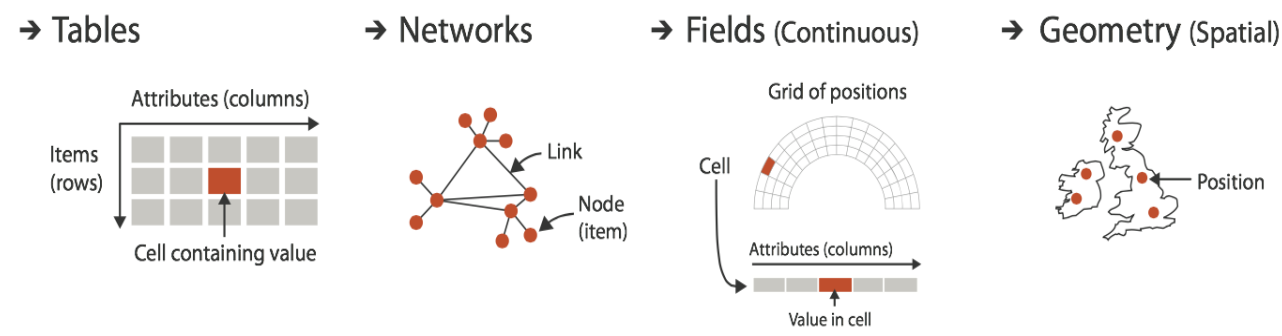

Figure 14: A summary illustration of dataset types and their types. Table and network have discrete values while fields has continuous values. Source: (http://www.cs.ubc.ca/ tmm/talks/minicou rse14/halfdaycourse14.pdf).

\subsubsection{Multiple Views}

The Coordinated and Multiple Views part of study is a specific exploratory visualization technique in which the data is represented in different ways that enables the users to understand and interact with the data better. According to Roberts, 2007, there are several reasons make the Coordinated and Multiple Views study more attractive. One of the reasons is that scientific experts are seeking to investigate complex data. These data may come from different datasets, which requires data aggregation and more analysis to generate comprehensive and useful information. The other reason is that human beings are always looking for things that they are familiar with. As a result, they will follow the system that they used to deal with previously even if it lacks the richness that other systems may offer. Therefore, it is favourable having a multiple viewing environment that shows the data in different displays to meet users requests. An example of a multiple-view system is MulteeSum, which is a tool for "comparative spatial and temporal gene expression data" as seen in Figure 15 Meyer et al., 2010. The figure shows that MulteeSum tool supports inspection and curation of datasets presenting gene expression over time. 


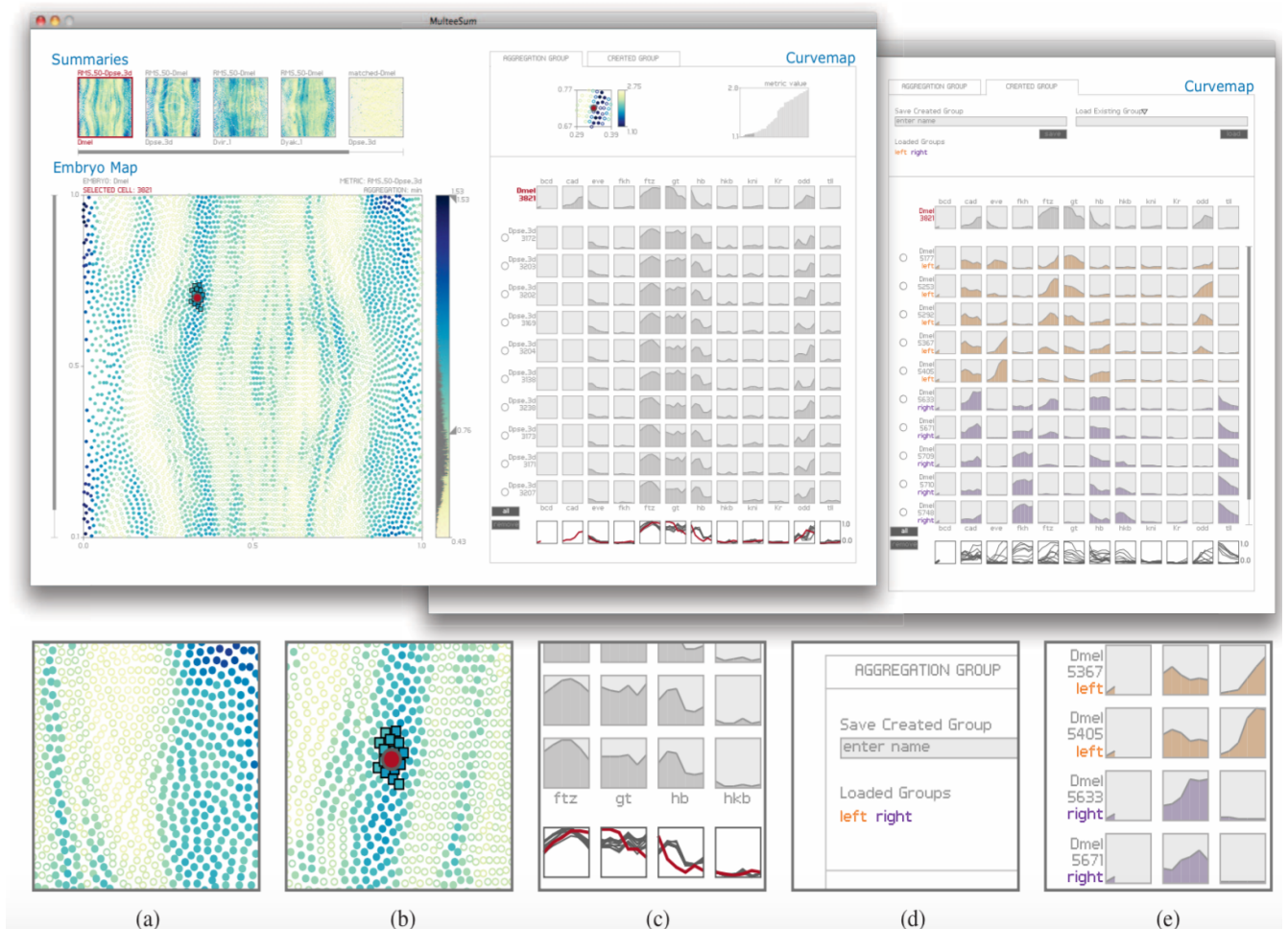

Figure 15: MulteeSum is a tool for Comparative Spatial and Temporal gene expression data. This tool supports inspection and curation of datasets presenting gene expression over time. This screenshot contains data for four species of Drosophila. (a) An embryo map where the summary value of a cell is encoded with colour. Circles are the filtered cells while the filled disks are the remaining cells. (b) The selected cell is coloured as red, while the other cells in the created group are represented as squares. (c) Curvemap highlights the cell's related curves in the overlapped plots. (d) Current groups loaded into the curvemap are given a colour. This is used when rendering a label for the groups. (e) The colour in the curvemap is used to indicate the group members' expression profiles. Credit: (

As any area of study, clear fundamentals should be stated as guideline or declaration for this area. Most of the demonstrations and the ideas for Coordinated and Multiple Views for Exploratory Visualization are taken from discussions and opinions that stated at Coordinated and Multiple Views Conferences. In Roberts, 2007 paper, a seven fundamental areas are covered. These fundamentals will be declared briefly, mainly from the above mentioned paper, to explain the available suggestions about how to build an efficient multiple-view system.

Data Processing and Preparation: One of the core challenges for exploratory visualization is dealing with enormous amount of data. The reasons for this difficulty are that huge dataset: takes long time to process, has many relationships, are slow to explore and the data may come from different sources. Therefore, the huge dataset needs to be simplified first before displaying. In order to make to perform simplification, data should be aggregated and cleaned from any possible 
errors. Most of the systems use some techniques to substitute the missing information by using average value; nevertheless the integration between these data fusion techniques and Coordinated and Multiple Views system still did not fully incorporated. For speeding up the running time for multiple views, many techniques are suggested such as parallel algorithms, data mining and grid based architectures. In addition, it is suggested that the liner algorithm should be avoided especially with large datasets. However, few researchers apply these recommendations to Coordinated and Multiple Views systems.

View Generation and Multiple Views: At the beginning of this part, view generation will be stated with mentioning its three common styles. Then the term of multiple-view and form will be declared with some examples.

One of the top questions that the Coordinated and Multiple Views system developer may ask is which style of visualization should be picked for viewing the system. Consequently, the developer will figure out how to map the information to this style. The other two questions that should be considered are: how to abstract this data to fit in the system and how the users will interact with the system.

When the users change one of the parameter, there are three reactions to this change. First possible reaction is the parameter modification will replace the information to new one. The second, a new window will be opened with the new information which called replication. The last scenario is the new information will be merged with the old one, which called overlay. Replication is one of CMV principle when the user can move from window to another.

Back to selecting a suitable style for interactive system, there are three categories for userinterface style. First, menus and buttons style in which the user pick the parameters from. Second, modular approach such as Module Visualization Environments. This style adapts the idea of giving users more flexibility by making them select the style from variety of offered modules. These modules will be linked together as multiple-view display after user selection. However, the coordination between these views is created using more command and modules. Third, using automatic algorithms from which the presentation is created by the users preferences. This style gives the user more flexibility for exploring the data but it increases the user task, which makes it not favourable in the developer's side.

After selecting the style, developers should think about how many forms are needed to be displayed in a single window. First we need to understand what is form and view meaning. Form means the way of representation such as maps, networks, charts, tables, matrix, symbols and glyphs. Multiform in another hand describes the data that displayed in diverse forms. In another hand, MultipleViews concept cares about the data that displayed in multiple windows beside each other in specific coordinates. These concepts can be merged in one system which called dual view system which may have variety of views such as: overview-detail, focus-context, difference views, master-slave and small-multiples. In overview-detail view, the data will be showed in one section and the details will be showed in another. If the dataset is huge, the overview should be abstracted and simplified first in order to represent the meaningful and useful information. Focus-context view is similar to overview-detail style in which the details will be showed in part while the context of the information 
in another. Difference views style is used to evaluate algorithms or underline textual differences. In this style different view styles is combined to show the differences. In master-slave style, one of the dual view part control the other part representation. Small-multiples style is not strict dual since multiple and small views come beside each other. This is usually used with symbols and glyphs that shown in matrix.

Exploration techniques: Changing the data processing, filtering the displayed data, changing the display color, zooming through the data in/out, and placing the view windows in specific coordinate all these are kind of user interaction with the system. There is a need for techniques to build such kind of systems. There are two common techniques that allow data exploration which are indirect and direct manipulation.

For indirect manipulation technique the users deal with following: menus, buttons and sliders. These tools used to indicate the type of interaction, if it is filtering the displayed data or any other kind of interaction. In direct manipulation technique, users themselves can do any type of interactions. This technique has two approaches, which are brushing approach and manipulators/widgets approach. These two approaches are supported in Coordinated and Multiple Views system in diverge levels. In brushing, if an element is highlighted in one side, the related information in other views will be highlighted too. This method enables the user to explore the data deeply. In the second approach, each manipulator/widget is connected to particular elements, and the users can change element properties directly using the widget.

Coordination and Control: The way that the views are organized and structured is following some theories. These theories explain how data relationships determine the place of the view. First, if a parameter change another view this means they are related, so they can grouped together which called render groups. Second, the views are related based on the early stage such as during data preparation and clustering, during mapping and rendering, or during manipulation stage. Third, the views coordination may change based on user exploration sessions.

Tools and Infrastructure: Developing an effective Coordinated and Multiple Views system is not easy job. It required having models and tools that guide the users to create the desired system. In this section, some of the most common models and tools will be mentioned briefly to give an idea about them.

Constraint approach, data centric approach and Model-View-Controller approach are the main three architecture models that connected directly with Coordinated and Multiple Views system building. In constraint approach, the system building goes through many small constraints or at least one. Therefore, the constraints are identified at the beginning, then rest of the system restructure around it. For example, putting specific presentation constraints, then building the whole system based on it. Data centralization as concept is coming from relational database in which any change in one component is reflected immediately on the others. The last approach is Model-View-Controller, where the internal representation of information is separated from the ways that information is 
presented to the user by splitting the software application into three communicated parts as seen in Figure 16 Deacon, 2009.

While creating a tool for Coordinated and Multiple Views system is still challenging, there are some toolkits and languages that can help to create such system by allowing developers to insert graphical displays to their applications.

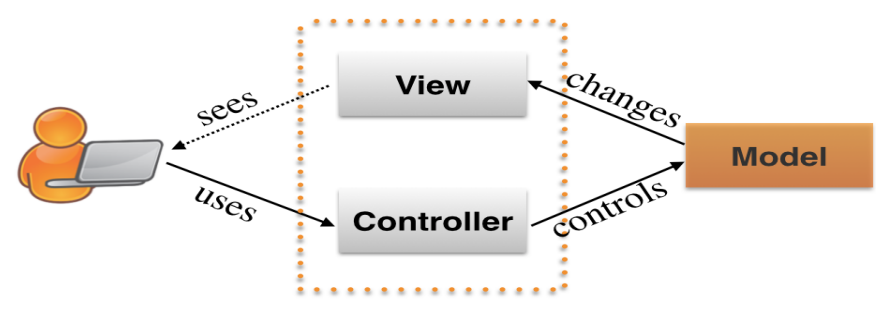

Figure 16: Model-View-Controller architecture where the software application divided to three communicated parts: model, view and controller. In this architecture, the internal representation of information is separated from the ways that information is presented to the user.

Human interface: Researchers started to pay attention to the importance of managing the users session and how they move from view to another. There are some tools, such as utilize dataflow modules, which enable storing users history session. However, these tools lack to the effective management. The screen size is an important factor that affects the user transferring between windows. The system is preferable to give the user the help by deleting unwanted windows or controlling where the windows is placed in different screens. Some systems use certain techniques to solve this issue such as zoomable user interfaces (ZUI's) and elastic windows. However, some of these techniques are not full joined with Coordinated and Multiple Views systems. Another solution to the large windows beside ZUI's is using big projected screens. Some companies propose a technology that operates multiple LCD panels to realize one large screen. However, these techniques not fully integrated with Coordinated and Multiple Views systems, and these screens still need a better navigation tools.

Usability and Perception: Usability is big issue in visualization area since many powerful systems are available but big question is: are they useable or not. Currently, researchers have started to act seriously and pay more attention to usability analysis. In Wang Baldonado et al., 2000 paper, there are some guidelines should be considered in developing multiple-view visualization systems, which are summarized into eight rules which are: diversity, complementarity, decomposition, parsimony, space/time resource optimization, self-evidence, consistency and attention management. In diversity, multiple-views should be used when there are diversity of models, user profiles, attributes, abstractions or genres. In complementarity rule, multiple-views should be used when the views have connections or differences. Decomposition rule cares about dividing complex visualizations into smaller views for easer management while parsimony rule cares about using multiple views 
slightly. In space/time resource optimization rule, developers should balance the space/time costs and benefits of presenting multiple views that displayed sequentially compared to side-by-side. Selfevidence cares about using perceptual cues that makes multiple-views relationships more obvious. Consistency rule states that developers should keep the multiple-views interfaces consistent. Attention management rule states that developers should use perceptual techniques that keep the user attentive on the right view.

\subsection{Whole Genome Visualization Tools}

As we mentioned previously, understanding huge amount of genomic data that resulted from sequencing and annotating genomes can be a challenge. Consequently, there is high demand for visualization tools for this data. There are some common available approaches that will be briefly discussed with some examples in this section, which are the genome browser, spreadsheets, network, circular and tree visualization tools.

\subsubsection{Genome Browsers}

Genome browser is a software that represents visually the enormous genomic data that gathered from specific databases for studying purpose. Since humans unable to understand the genomic data textually these browsers are designed to give users the ability to access and display sequence data in organized way. Most of the browsers are built in coordination system where the gene features aligned to the genome. For each view, users can display portion of the genome and scale by zooming in and out for controlling the amount of the displayed data. Ensembl (http://www.ensembl.org), JBrowse (http://jbrowse.org) and GBrowse (http://www.gbrowse.org) are some of the most common available browsers that used to visualize genomic data. For more examples about genome browsers, see the attached catalog in Appendix A about visualizing gene model in genome browsers. In this section, we will examine one of most common existing browsers which is UCSC Genome Browser.

University of California Santa Cruz (UCSC) is developed and supported by the Genome Bioinformatics Group at the University of California Santa Cruz (UCSC) http://genome .ucsc .edu. This browser is using the human genome assembly that is generated by NCBI. It has variety of living groups to pick from such as mammal, vertebrates, insects, nematodes, yeast etc. UCSC site has features that makes it useful browsing tool, such as easy navigating, ability of browser configuring, and data extracting. It allows the users to access the gene region and download its genomic sequence in easy-going steps. In UCSC browser, the users also can control the amount of detailed information that displayed in the browser. UCSC browser has many tracks option that enable displaying mRNA and expressed sequence tag alignments, multiple gene predictions, sites, transposon repeats and more Kent et al., 2002.

To browse the genome, the user sets first the group, genome, assembly and position choices. Then, the user can submit it immediately, or configure the available tracks, or can add some custom 
tracks by providing annotation data in some file formats such as GFF or BED format; then the user can submit the configuration to the site. Below in Figure 17 is an example that shows Rat genome on UCSC Genome Browser. In this figure, a small portion of the rat genome is displayed where the user can zoom in/out for more or less details from chromosome called chr1. There are only two chosen tracks to be displayed, which are other RefSeq and RefSeq genes. Other RefSeq track shows the known protein and non-protein coding genes for organisms other than rat. RefSeq genes track shows known rat protein and non-protein coding genes taken from the NCBI RNA reference sequences collection (RefSeq).

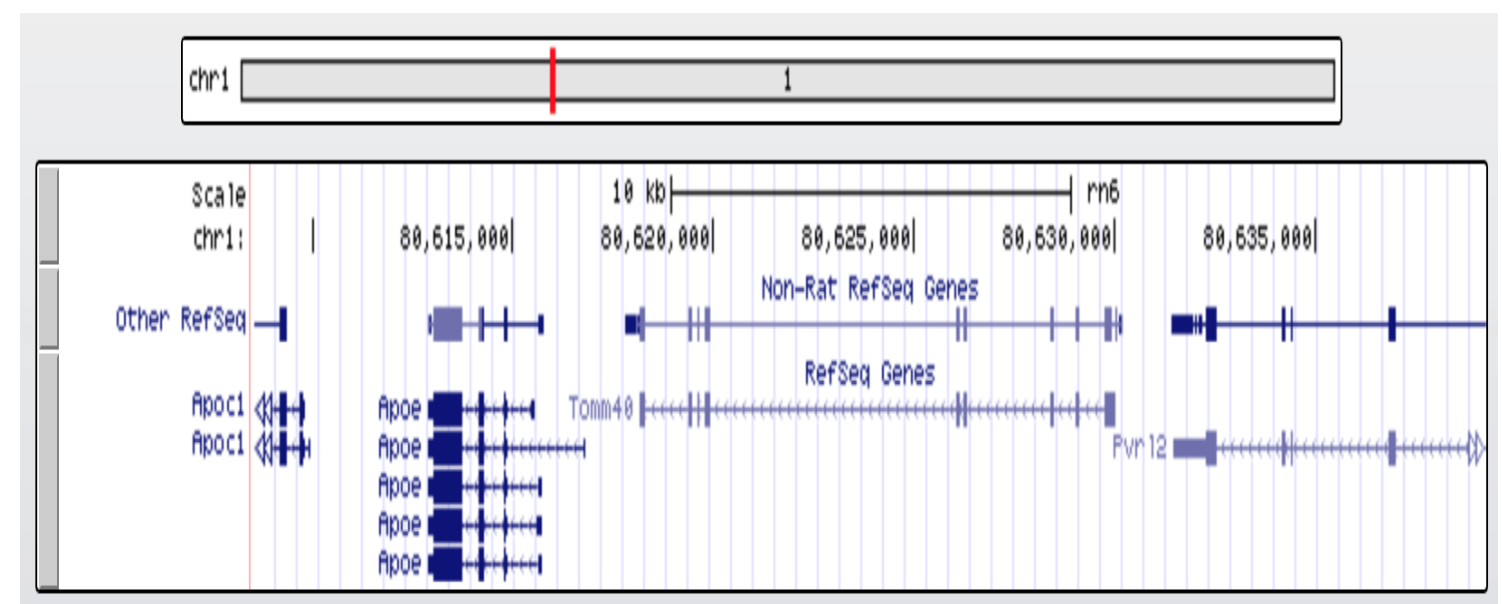

Figure 17: Rat genome on UCSC Genome Browser in Jul. 2014 (RGSC 6.0/rn6) Assembly. A small portion of the chromosome chr1 on rat genome is displayed here which is from the position 80608553 to 80639261 . There are only two chosen tracks to be displayed from chromosome called chr1 which are Other RefSeq and RefSeq genes. Other RefSeq track shows the known protein and non-protein coding genes for organisms other than rat. RefSeq genes track shows known rat protein and non-protein coding genes taken from the NCBI RNA reference sequences collection (RefSeq). For each gene model on both tracks, the small rectangles are the exons.

\subsubsection{Textual Spreadsheet}

For tabular (or spreadsheet) data representation in biology, there are some efforts that are done. Some of them are developed for the purpose presenting annotation data textually such as UCSC Table Browser (http://genome.ucsc.edu) and MaizeGDB (http://www.maizegdb.org), while others developed for of data exchange such as ISA-TAB.

UCSC Table Browser is mainly used for retrieving and manipulating data that associated with a track in genome browser in textual format. Having a table that has DNA sequence or annotation data for the entire genome for specific coordinate and having some statistics about selected dataset are some of the benefit of using UCSC Table Browser. for visualization sample of these tables, see Appendix A

The Investigation-Study-Assay (ISA) tab-delimited (TAB) format is developed initially to be a 
universal purpose framework for collecting and exchanging complex experiments metadata such as protocol and sample characteristics. These data particularly is related to "omics-based" experiments such as genomics and proteomics, but it is not limited to them. In Figure 18 below, is a sample from ISA-TAB file for protein expression profiling data, which visualized after using ISA-TAB web viewer. In ISA-TAB, data are organized in tab delimited file which can be viewed as textual table representation using ISA-TAB web viewer for ISA-Tab files that found in (https://github.com/I SA-tools/ISATab-Viewer).

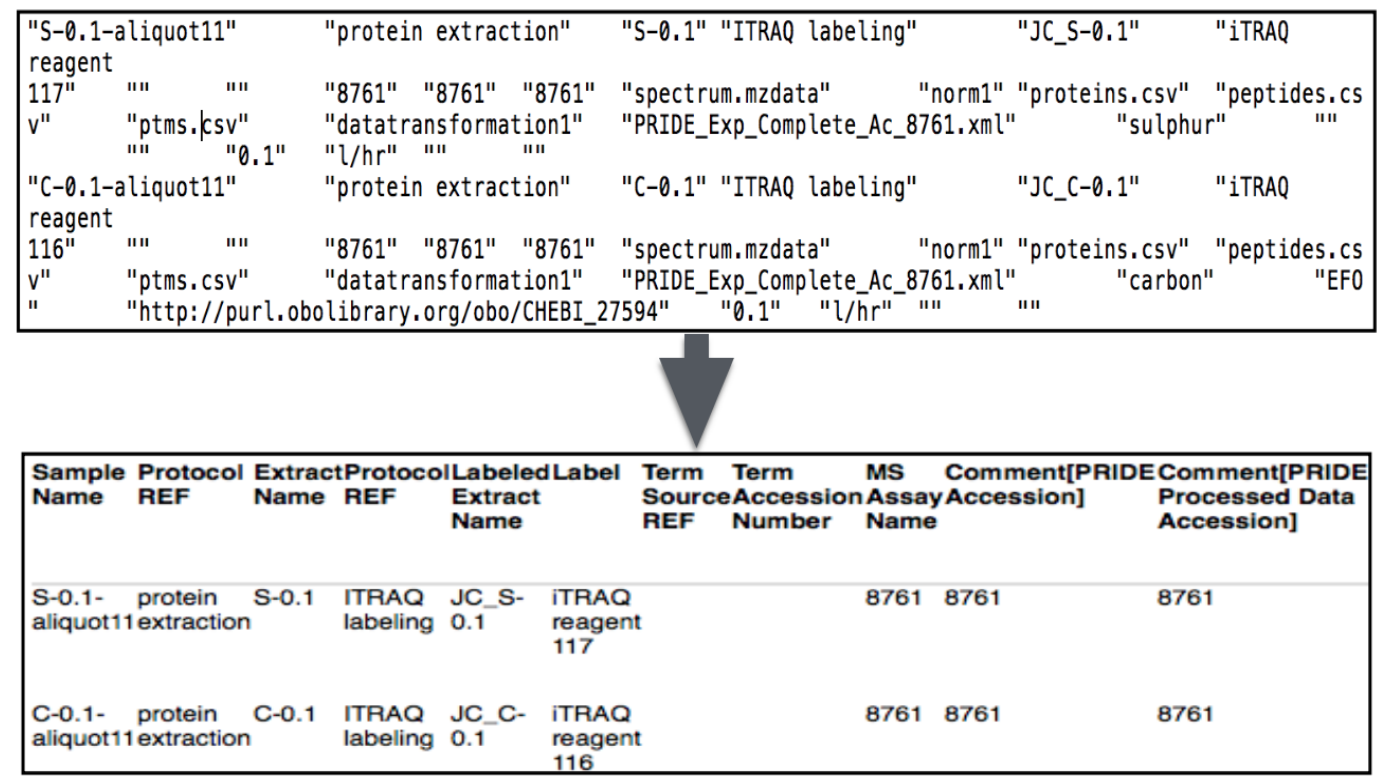

Figure 18: Protein expression profiling mass spectrometry data that saved in ISA-TAB file format. In ISA-TAB, data are organized in tab delimited file as seen in the box above. This tab delimited file can be visualized as tabular format using ISA-TAB web viewer as seen in the box below.

\subsubsection{Network Visualization Tools}

In general, networks represent relationships and in biology these relationships could be functional or physical relationships. These include protein interaction, gene-gene interaction, regulatory transcription factors that regulate the genes interaction, or having a shared protein domain or family. The most benefit from using molecular interaction networks is to discover huge dataset, which helps later for understanding gene function in biology. It is more powerful method than tables to express many relationships among these massive datasets [Pavlopoulos et al., 2008]. Many network visualization tools are available to visualize biological interactions. These tools are varying on their public availability and user friendliness especially when thousands of circles with many connections have to be visualized and explored Pavlopoulos et al., 2008.

One of the most common network visualization tools is Cytoscape. Cytoscape (http://www.cy toscape.org) is an open source network visualization and analysis tool for bioinformatics, which 
enables users to display networks through the web. It is mainly used for biological research applications with many plugins that used to show specialized features [Shannon et al., 2003. It is useful tool to visualize molecular interaction networks and biological pathways. In addition it integrates these networks with extra information such as gene expression profiles and annotations. Cytoscape works by loading network data then visualizes it as nodes (usually circles) and the edges as interaction between them. In Figure 19 below is an example of using Cytoscape in visualization of protein interaction networks Gehlenborg et al., 2010. In this figure, a network for Mycoplasma pneumoniae protein interaction data derived by mass spectrometry analysis. In the left network, there is an initial protein interaction network which has $>400$ proteins. Each node has a color that represents different data. In the right network, there is recomputed network remaining after removal of nodes not of interest. Nodes here are coloured according to functional annotation and their shapes represent different roles. In both networks, each node has relationship with other node if they are connected by link.
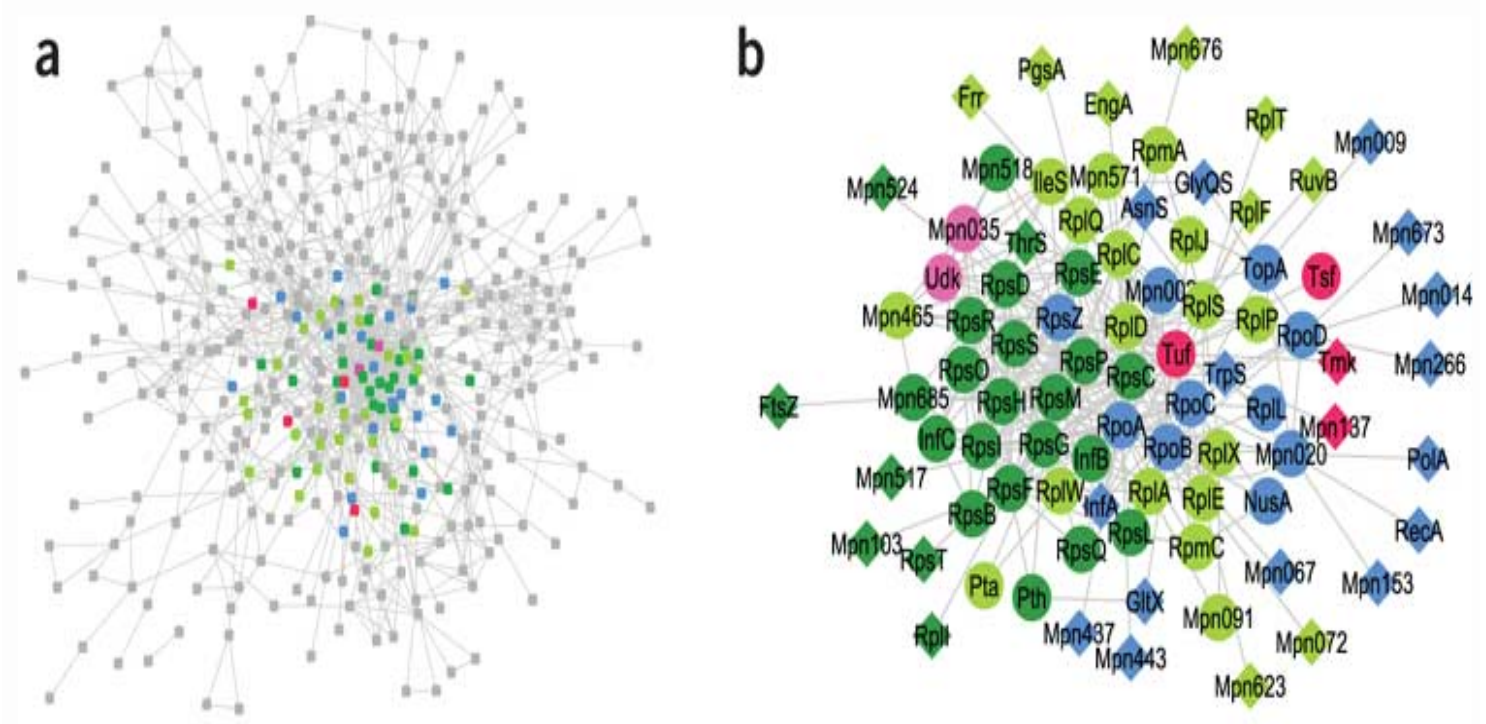

Figure 19: Cytoscape images for Mycoplasma pneumoniae protein interaction data derived by mass spectrometry analysis. (a) Initial protein interaction network ( $>400$ proteins). Each node has a color that represents different data. For example, blue represents RNA polymerase; dark or light green represents small or large ribosomal subunits respectively; red represents elongation factor. (b) Recomputed network remaining after removal of nodes not of interest. Nodes are coloured according to functional annotation and their shapes represent different roles. Circle shape represents core protein of complex while diamond represents protein attached to complex but not part of the core. Credit: Gehlenborg et al., 2010. 


\subsubsection{Tree Layout Visualization Tools}

Tree layout is commonly used in the application that needs an efficient arrangement for biodiversity information. It is the suitable way to present the phylogenetic context. By using the tree layout, a better understanding for organism evolution patterns in gene and genome can be achieved Soltis and Soltis, 2003.

Interactive Tree Of Life (ITOL) (http://itol.embl.de) is one of the well-known systems adapted this kind of visualization. It is web-based tool for displaying phylogenetic trees with adding customization features such as pruning and collapsing branches, downloading the tree in different formats, configuring and uploading dataset from text file to the tree Letunic and Bork, 2007 Letunic and Bork, 2011. The created tree can be represented in circular, normal and unrooted (radial) modes as shown in Figure 20. In this figure a tree in three different modes are showed to represent 191 species from Archaea, Bacteria and Eukaryota in green, purple and pink respectively. Each mode has different settings such as rotation and arc options in circular and unrooted mods. The circular made is useful to display mid-sized trees that have several thousand leaves.
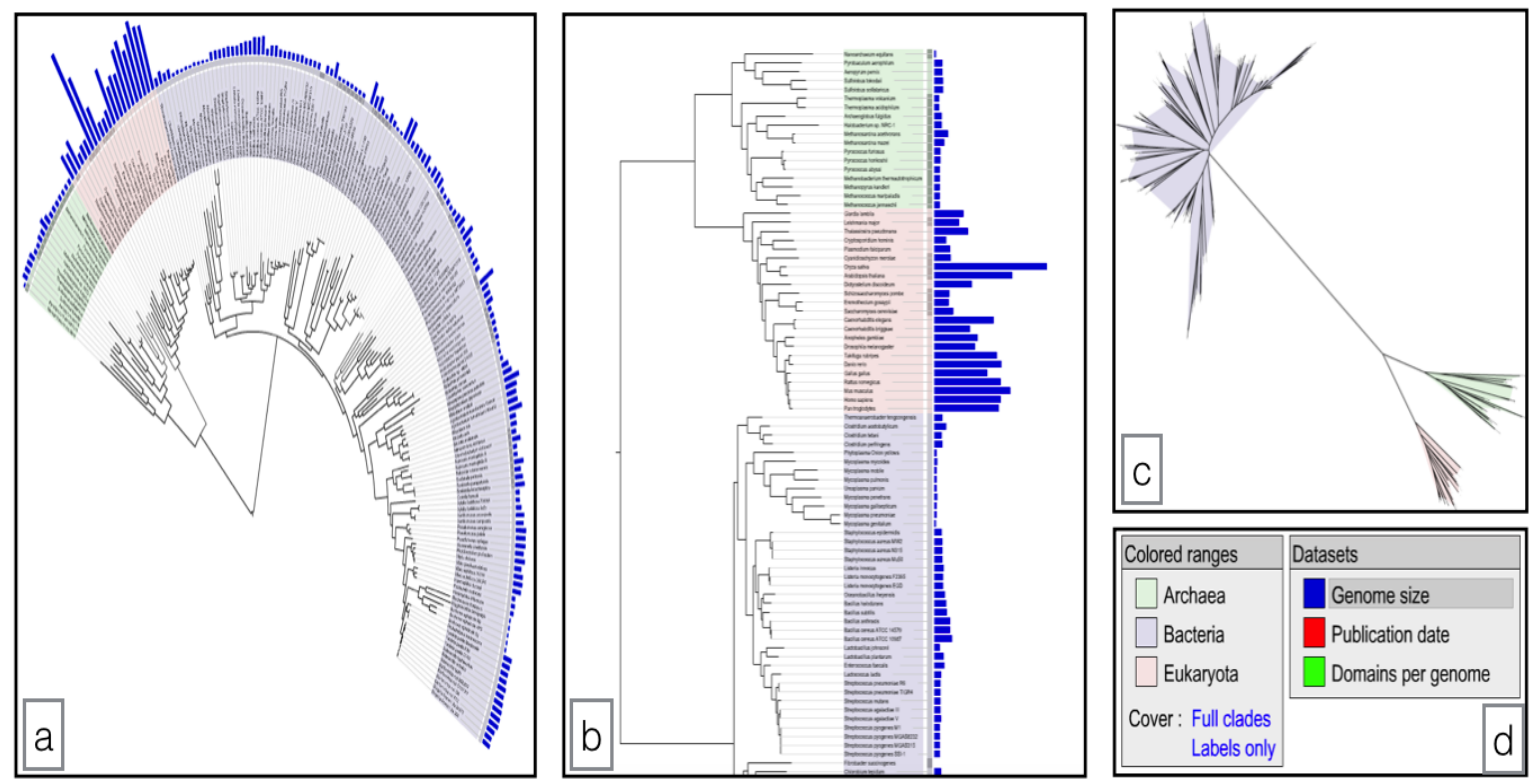

Figure 20: Representing phylogenetic tree in iTOL in different modes: circular, normal and unrooted respectively. The colours represent different type of data as seen in the small box (d) in the bottomright. a) A tree has 191 species from Archaea, Bacteria and Eukaryota in green, purple and pink respectively. The tree here is presented in a $180^{\circ}$ arc $\left(350^{\circ}\right.$ is the default). The blue bars represent the genome size dataset. b) The same tree represented in the normal mode where labels are aligned to the leaves. c) The tree in unrooted (radial) display mode with $360^{\circ}$ arc by default. 


\subsubsection{Circular Layout Visualization Tools}

Circular Layout is practical for exploring relationships between objects especially when the tabular layout fails to express them in single view. One of the common interactive systems that uses circular layout visualization is Circos (http://circos.ca/intro/circular_approach/). It is software that creates composite circular display of genomic data and multi-layered annotations such as visualizing alignments, conservation and chromosomal relationships as seen in Figure 21. In this figure, wholegenome RNA-seq data is showed. The data is represented in six tracks which are cytoband, genomic location of top 100 genes, genome-wild RPKM, gene location related to placenta in the OMIM, enriched genes in placenta and functional clustering of unique genes. Each one of them represents different data. Circos is commonly used in genomics and cancer biology, although it can illustrate several types of data. There is a comparison between tabular and circular layout that can be found in (Circos and tabular visualization).

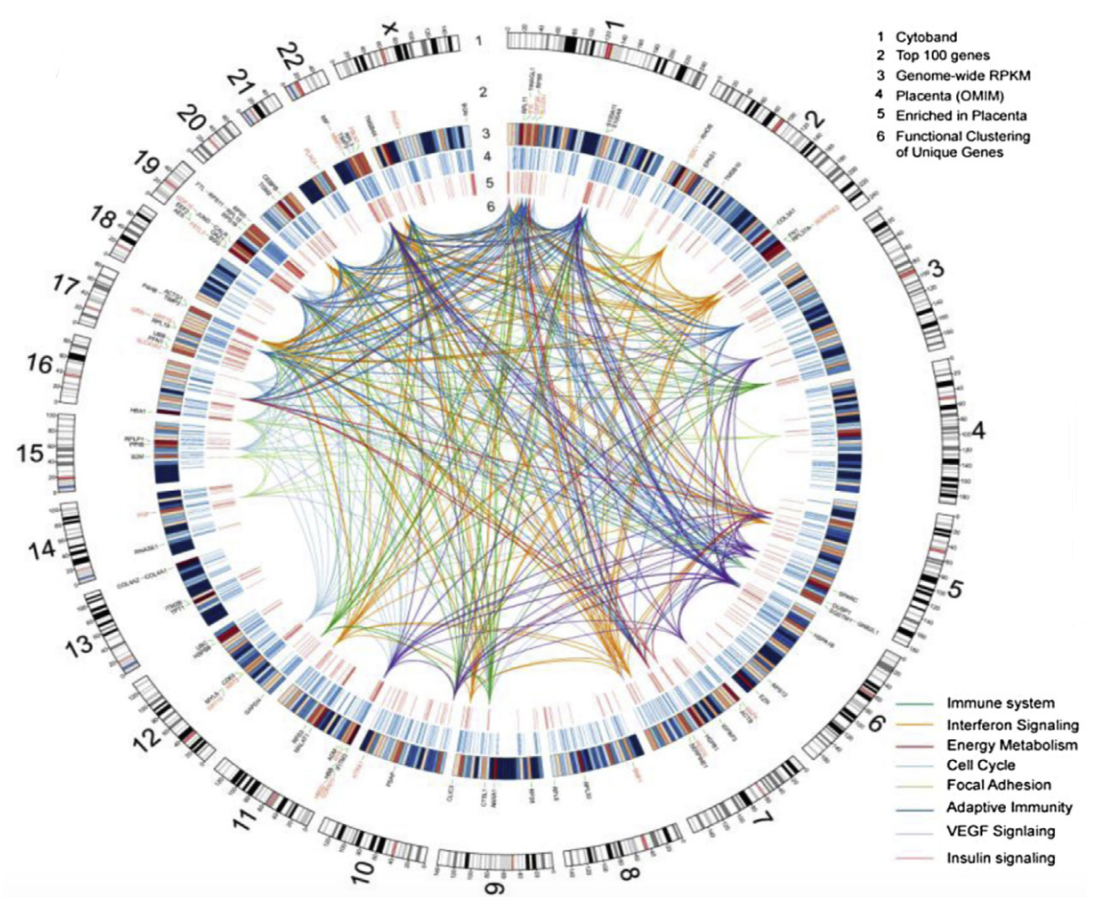

Figure 21: Using Circos Circular layout to express genomic annotation data for comprehensive analysis of the human placenta transcriptome. The data is represented in six different tracks. Track 1 represents cytoband where chromosomes are represented qter to pter. Track 2 represents genomic location of top 100 highly expressed genes in placenta based on average RPKM values. (Gene names in red represent enriched genes in placenta). Track 3 represents average RPKM values summarized over $6 \mathrm{MB}$ regions showing regions of high gene expression. Track 4 represents locations of genes related to placenta in the OMIM database. Track 5 represents enriched genes in placenta (3-fold over 7 other tissues); Track 6 represents biological functions enriched among placenta-enriched genes. Credit: Saben et al., 2014] 


\subsubsection{Related Works}

\section{FancyGene}

FancyGene is a web-based software that represents the gene structure in graphical way based on genomic coordinates of one ore more genes. Users can dynamically add some features after the standard representation of the gene structure is shown. These features may include adding the architecture of protein domains and changing the appearance of each object using a simple web interface.

The software can generate two output formats. First, high resolution PNG format that can be used in two ways: screen and poster presentations. Second, portable document format (PDF) that can be edited to allow the user to generate high-quality images suitable for publications Rambaldi and Ciccarelli, 2009. In Figure 22, gene representation for locus on human chromosome 17 taken from FancyGene. The figure also shows the option field where the user can set and change some of displaying options such as changing the colour of the exon box.

FancyGene has several features. First of all, it accepts data in a many input formats such as GFF (General Feature Format) and GTF (Gene Transfer Format) files. FancyGene is able to accept a simpler format where each line contains gene exons, the start and end points. The introns will be computed automatically. Labels to define the direction of transcription and to add UTRs information also can be added. Once input file is uploaded, it will be converted into the FancyGene format where each line contains four mandatory fields: the gene name label, the object tag, the start and stop of the object. The object tag can be intron, exon, UTR, marker or domain. The user can save the input file and the configuration file to re-use them later to create the same layout for the locus of interest. The second feature of FancyGene is having the ability to represent all parts of the gene structure that labeled in the input file such as coding exons, introns, UTRs and several types of labels. Third feature of the software is the ability to project protein domains information on the gene structure by associating exons to the encoded domains. FancyGene automatically alters the coordinates of the protein domain amino acidic into nucleotide coordinates. Then, it covers the coding exon by the domain architecture. Final feature is that the users have the ability to add features and new genes. They can also modify the look of any object and change their order such as changing gene labels positions. The coordinates of the locus also can be improved and the background can be changed [Rambaldi and Ciccarelli, 2009].

\section{FeatureStack}

FeatureStack is one of the Perl modules that used for automatic generation of multi-gene images. This software can be used to take BioPerl-compliant gene or transcript features as input and provide them on top of each other using a user-defined BioPerl glyph. The user has the option to generate SVG or PNG format for the output images. In addition, FeatureStack comes with a new BioPerl glyph and decorated_gene, which is able to highlight protein features on top of gene models Frech et al., 2012. According to Frech et al., 2012], the goal of the FeatureStack is to provide maximum flexibility in image generation. This is done by providing some options and enabling some parameters 


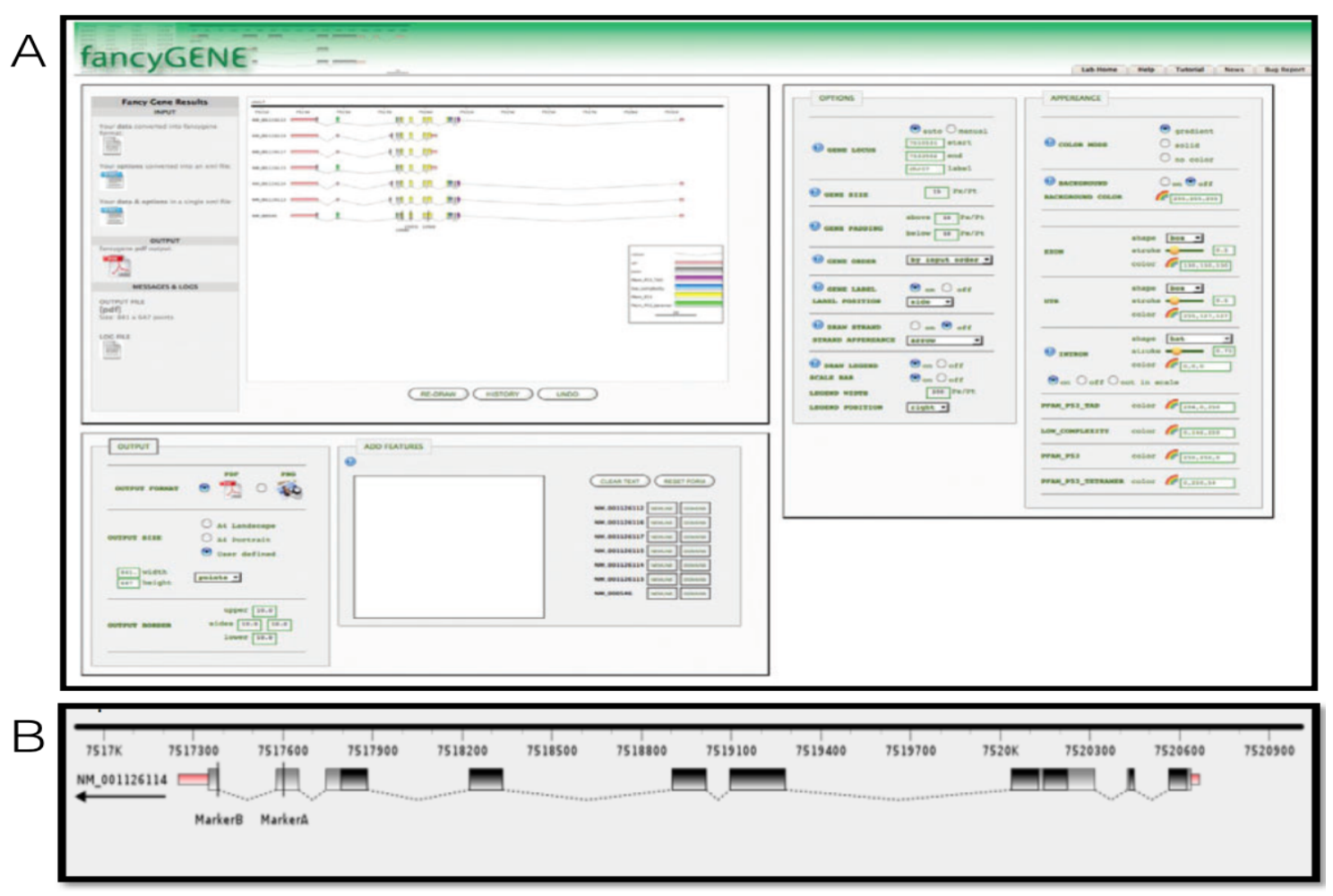

Figure 22: A) Screenshot of FancyGene website which has a gene representation for locus on human chromosome. The locus on human chromosome 17 corresponding to the p53 gene (entrez id: 7157) is shown. Credit: Rambaldi and Ciccarelli, 2009. B) Gene structure elemnts where intron represented as dash, exons represented as grey rectangles and encoded protein domain represented as black and grey rectangles.

settings to have better control for all aspects of the rendering process. One of FeatureStack advantages is that the user can use it with any BioPerl glyph that is compatible with the input features' structure. These glyphs will be more powerful when combined with FeatureStack decorated_gene glyph. The combination between them can generate rapid and automated large and annotation-rich images of stacked gene models. The comparison of gene structures will be much easier, because decorated_gene allows the highlighting and labeling of protein motifs like signal peptides, transmembrane domains, or protein domains on top of gene models. In Figure 23, RFX transcription factor gene family are represented by FeatureStack where the genes are ordered by their phylogenetic distance. They are also horizontally aligned by the their most conserved feature which is the DNA-binding domain that drown in black. By default, both exons and introns are drawn to scale, but exons in this figure is scaled while the introns has fixed size of $50 \mathrm{bp}$. 


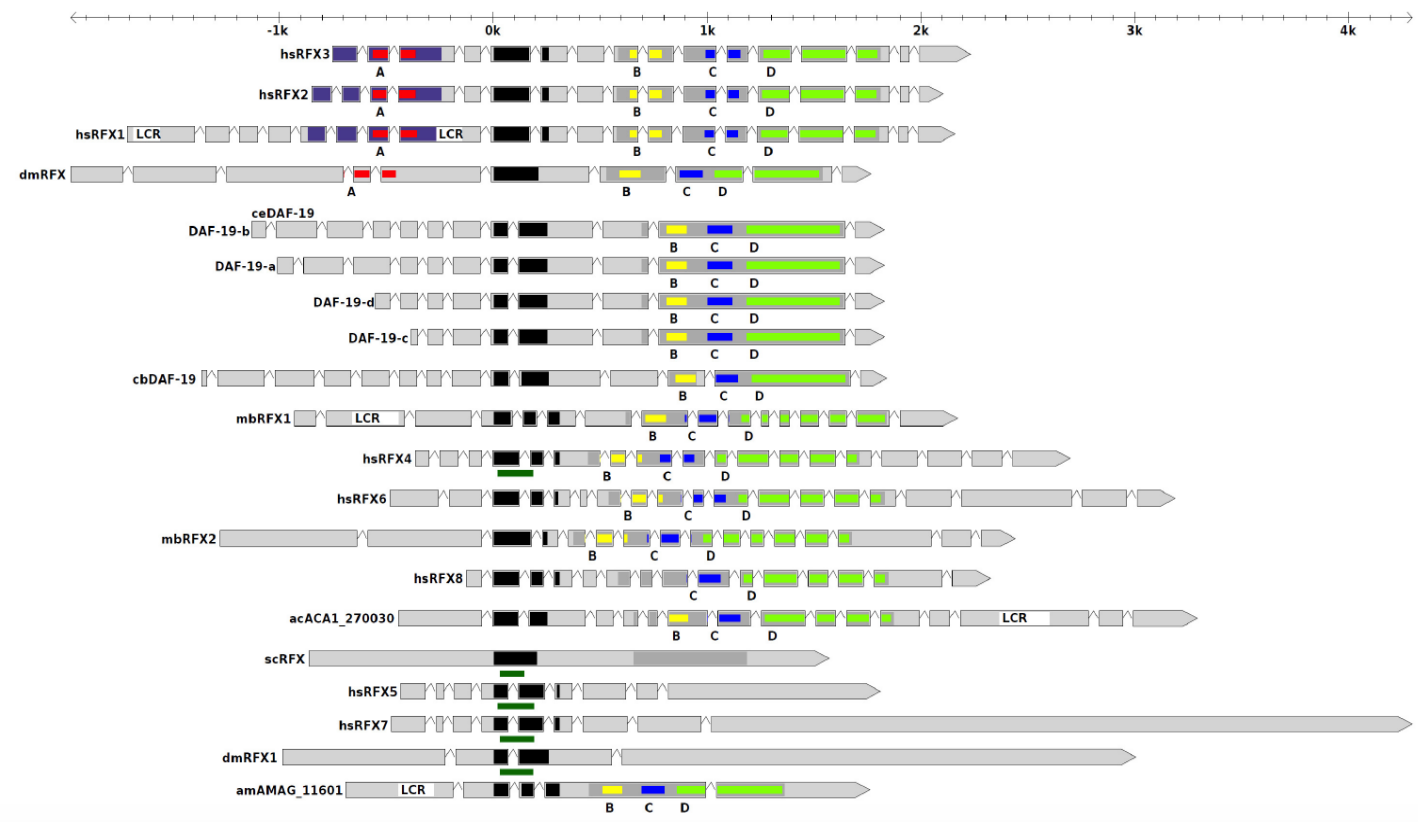

Figure 23: FeatureStack output which represents RFX gene family members over a different species: human (hs), fly (dm), C. elegans (ce, 4 isoforms), C. briggsae (cb), M. brevicollis (mb), A. castellanii (ac), S. cerevisiae (sc), and A. macrogynus (am). The genes are ordered by their phylogenetic distance. They are also horizontally aligned by the their most conserved feature which is the DNAbinding domain.Domains are drawn in different colors: DNA-binding domain (black); N-terminal activation domain (dark slate blue); A, B, C, and D domains (red, yellow, blue, and green, respectively); combined BCD domain (dark grey); low complexity regions (LCR) in white; dark green bars below DBD indicate regions of similarity with viral Pox_D5 domain. Credit: Frech et al., 2012]. 


\section{Chapter 3}

\section{CGene and CGenome: System Analysis and Design}

In this chapter we will see what services the system offers, what requirements our system should have, how the system is built, and what data format and technology are used to construct the system.

\subsection{Project Scope and Objectives}

In our research, we are concerned with the development of a visual system offering the possibility to produce a visual HTML5 spreadsheet for whole genome, in which the visual images are Scalable Vector Graphics (SVG) images for gene annotation features. The main services of the system are:

- Creating HTML5 spreadsheets.

- Browsing the spreadsheets.

- Presenting SVG images for both spreadsheets. For CGene spreadsheet, Gene model and Protein domain architecture images are presented. For CGenome spreadsheet, Gene location, Go Slims bitmap and Go Slims histogram images are presented.

- Downloading the SVG images.

- Selecting a dataset from the spreadsheet.

\subsection{System Activity Diagram}

Generally, an Incremental Method is used to develop the webpage starting from collecting the data from AspGD database, analyzing them, coding then testing them one at a time to make sure that the system objectives are achieved. Figure 24 shows the system activity diagram used to accomplish the objectives in Section 3.1. As shown in this figure, the first step is collecting the files from the AspGD 
database (www.aspgd.org) about Aspergillus fungal genomes with different file formats. Those files include gene features, Protein Domain Predictions information, Gene Ontology (GO) annotations, AspGD chromosomal features and Go Slims description. Then, the system analyzes these files to extract the key features that required for displaying the following features: gene model, protein domains, genes location in the chromosome and GO Slims availability in the genome. After that, a scalable vector graphics (SVG) images are created for these features. Then, multiple HTML5 pages are created which include the master page, CGene pages and CGenome page. When the master page is displayed to users, they can select the link to any genome spreadsheet in CGene or select the link to CGene page. In both spreadsheets, CGene and CGenome, the users can brows the spreadsheet, select row dataset and download the SVG images from the spreadsheet.

\subsection{General Requirements}

In general, the system should reflect the biology concepts. The system components such as a gene model should harmonious with the existing visualizations. Consequently, it does not require much time and effort from the user to understand the system.

\subsubsection{Functional Requirements}

\subsubsection{Functionality of CGene}

CGene, pronounced as See-Gene, is an HTML5 web-based spreadsheet, the data from a single genome will be explored and analyzed interactively as a visual spreadsheet. Each row in the visual spreadsheet captures the information about the gene and protein as text, images, and numerical data. The requirements for the visual spreadsheet using HTML5 for whole genome display are:

1. The system shall analyze different file formats that collected from the AspGD database (www. aspgd.org) about Aspergillus fungal genomes such as standard GFF3 files, standard output files from InterProScan. Those files include data about gene features and Protein Domain Predictions information that used for displaying the following features: gene model, protein domains.

2. The system shall produce a spreadsheet.

3. Each row on the spreadsheet shall hold the information about one gene.

4. For each gene, the following columns: gene ID, gene length, gene-model, protein-length and protein domain architecture shall be available.

5. If the gene is not associated with a protein, the system shall put blank in the domain architecture cell and in protein length cell.

6. The system shall present the gene ID as text.

7. The system shall present the gene length and protein-length as numbers. 


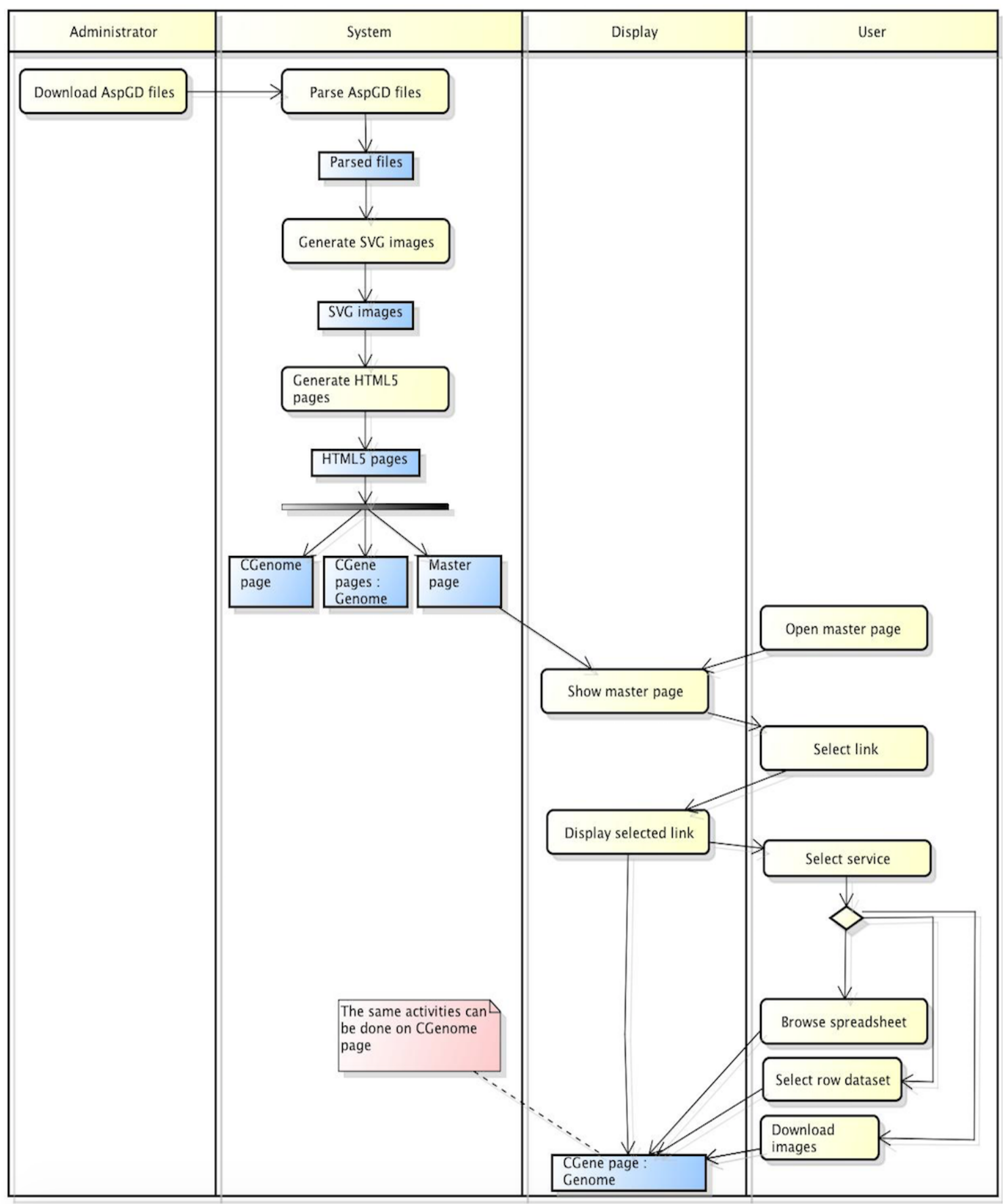

Figure 24: Activity diagram for visual HTML5 spreadsheets system: CGene and CGenome. The first step is collecting the files from AspGD database (www.aspgd.org) about Aspergillus fungal genomes. After that, the system extracts some key features that required for displaying gene model, protein domains, genes location in the chromosome and GO Slims occurrence in the genome. Then, a scalable vector graphics (SVG) images are created for these features. Then, multiple HTML5 pages are created which are the master page, CGene pages and CGenome page. The users can select a link from the master page to any CGene genome spreadsheet or select the link to CGenome page. For both spreadsheets, users can brows the spreadsheet, select row dataset and download the SVG images from the spreadsheet. 
8. The system shall present the gene model and protein domain architecture as Scalable Vector Graphics (SVG) format.

9. The system shall enable the user to select a dataset from spreadsheet rows.

\subsubsection{Functionality of CGenome}

CGenome, pronounced See-Genome, produces a visual spreadsheet where the data comes from multiple genomes. Each row in the visual spreadsheet captures the information about a single genome.

The requirements for CGenome are:

1. The system shall analyze the annotation statistics extracted from AspGD DB. The available data are for four genomes, which are A. nidulans genome, A. fumigatus genome, A. niger genome and A. oryzae genome.

2. Each row on the spreadsheet shall represent the information about one genome.

3. For each genome, the following columns: Genome ID, Gene Location, GO Slims Bitmap and GO Slims Histogram shall be available.

4. The system shall present Gene Location, GO Slims Bitmap and GO Slims Histogram as Scalable Vector Graphics (SVG) format.

5. In the Gene location cell, the system should show the genes location on each chromosome.

6. In the Go Slims bitmap cell, the system should represent the GO slims occurrence in the specified genome.

7. In GO Slims histogram cell, the system should represent the number of occurrence of each GO Slim term.

8. The system shall enable the user to delete rows.

\subsection{Domain Model}

Before describing our system in details, we need to explain a domain model for system components since this step is "the most important artifact" to generate during object-oriented analysis Larman, 2012]. This step will illustrate the meaningful conceptual classes in our problem domain. In this section, the most common conceptual classes in the system that reflects the real world will be defined. Then, the domain model diagram will be drawn.

\subsubsection{Gene and Gene-Model Conceptual Domain Model}

In order to build the Gene and Gene-Model conceptual domain model, we need to make a list of the important components that reflects the real-world conceptual classes. 
As mention previously in Chapter 2 Section 2.1.1 a cell is the basic building unit of creatures and living species. Each of the cells has the complete human genome on its nucleus, which composes of 23 pairs of chromosome. Each chromosome have a collection of DNA(Deoxyribonucleic acid) molecules that encode the genetic code which used in building and functioning of the human being and the different organisms Majoros, 2007. DNA contains many Genes. Gene is the fundamental unit of inheritance, comprising a segment of DNA (or RNA in some viruses) that codes one or several related functions and occupies a fixed position (locus) on a chromosome. Generally, genes are made of three kinds of nucleotide sequence which are: Series of exons (coding regions), introns (noncoding regions) and Regulatory regions (Promoter). Since gene is represented by gene model, there is a need to define some of the components that most well known genomic browsers use to represent gene-model (see Appendix $\mathrm{A}$ for genome browsers examples). Exons are segments of gene that are represented in the mature RNA product while introns are segments of DNA in a gene that connect the exons, but are removed from the transcript by splicing. Coding region is DNA sequence region of a gene that will be translated later into a protein. This region is started with start codon and end with stop codon. Start codon is a place that spots the start site at which translation into protein sequence begins while stop codon is a place that spots the end site at which translation into protein ends Majoros, 2007. The gene also has untranslated regions (UTRs), which are three-prime untranslated region ( $3^{\prime}$ UTR) and five-prime untranslated region ( $5^{\prime}$ UTR). The $\mathbf{3}^{\prime}$ UTR comes after the stop (termination) codon and located at the $3^{\prime}$ end of the transcript. The $\mathbf{5}^{\prime}$ UTR comes before the start codon and located at the $5^{\prime}$ end of a transcript.

After listing the important components that most well known genomic browsers use to represent gene-model, we create the domain conceptual model for visualizing these concepts as shown in Figure 25. In this figure, the components that reflect the real-world conceptual classes and their relationships are illustrated. This diagram has the basic building unit of creatures that called cell, which consists of DNA that found in chromosome. The DNA is transcribed into pre-mRNA. The pre-mRNA is spliced into mRNA, which is translated later to protein. The chromosome consists of multiple genes from specific genome. There are many components that the gene may include such as exons, introns, coding regions and UTRs. Gene model is the visual representation for the gene and its components.

\subsubsection{Protein and Protein-domain Architecture Conceptual Domain Model}

To build the Protein and Protein-domain architecture domain model, we need to make a list of the important components that reflects the real-world conceptual classes.

Protein, as described previously in Chapter 2 Section 2.1.1, is a large biological molecule, which its primary structure is the sequence of one or more long amino-acid residues. Amino Acids Sequence is the unique chain of amino acids that characterizes a given protein. They put together into a polypeptide chain on the ribosome during protein synthesis. A protein sequence is represented as a chain of 20 English alphabet letters (A,C,D,E,F, G,H,I,K,L,M,N,P,Q,R,S,T,V,W,Y) where each letter represents an amino acid. This Amino Acids Sequence may have many patterns and repeats. Patterns are regions that consist of a few amino acids. When these regions are 
identified in multiple sequence alignment process, it helps to recognize some sequence features such as the active sites of enzymes and binding sites EMBL-EBI, 2014. These alignments can be used to define either evolutionary or functional relationships Hertz and Stormo, 1999. Many proteins have runs of single amino acids that called repeat Albà et al., 2007. These repeats have specific roles in protein function and evolution. For example, repeats with complex patterns such as leucinerich and $W D$ mostly refer to the functional domain repeats, which are commonly participate in protein-protein interaction Luo and Nijveen, 2013.

As stated previously in Chapter 2, Section 2.4.1, protein structure is divided into three or four levels structure based on the references that categorized the protein structure. These structures are primary structure, secondary structure and Tertiary Structure. The next level of protein structure after amino acid sequence is Protein Structural Motif . It called super-secondary structure, which is defined by the connectivity between secondary structure elements. Helices and sheets are the most common secondary structures motif, but there are irregular secondary structure motifs that can have critical role in biological function Rangwala and Karypis, 2010. The most common structural motifs are Alpha Helix, Beta Sheets, Coil(or Loops) and Turns. Tertiary structure is the global 3D shape that represents the protein in three dimensional crystal coordinate. This shape contains of a variety arranged secondary structure elements that bonds together. There is a stable form of the tertiary structure which called protein folds. Protein folding is the process by which a protein structure assumes its functional shape or conformation. It is the physical process by which a polypeptide folds into its characteristic and functional three-dimensional structure from random coil Wikipedia, 2014.

Protein Domain Architecture, or domain arrangement, refers to the domains and their liner arrangements in individual protein. The direction of these domains goes a long the amino acid chain from N- to C- terminal Morrison, 2013. Domain architecture has many elements such as sites, motifs and domains. Site consists of the residues at specific position while motifs simply define the structural characteristics in a protein Biologyreference., 2014. Sequence motifs share a certain sequence of amino acids where a specific arrangement of amino acids that found in one protein can be found in other proteins. Motif can be part of domains such as the leucine zipper motif that frequently found as segment of a dimerization domain in several transcription factors. Domains consider as the basic building block of a protein structure. Usually they are responsible for a particular function or interaction, contributing to the overall role of a protein.

After listing the important components, we create the visualizing concepts in Figure 26. In this figure, the components that reflect the real-world conceptual classes and their relationships are illustrated. This diagram shows the protein, its structures, its domain representation and their relationships. at the beginning, the protein is structured into primary structure which is the amino Acids Sequence. This sequence has many patterns and repeats. The primary structure encodes protein secondary structure that encodes tertiary structure. Protein Structural Motif which called super-secondary structure consist of the common structural motifs which are Alpha Helix, Beta Sheets, Coil(or Loops) and Turns. Domain architecture represents many protein elements such as sites, motifs and domains. 


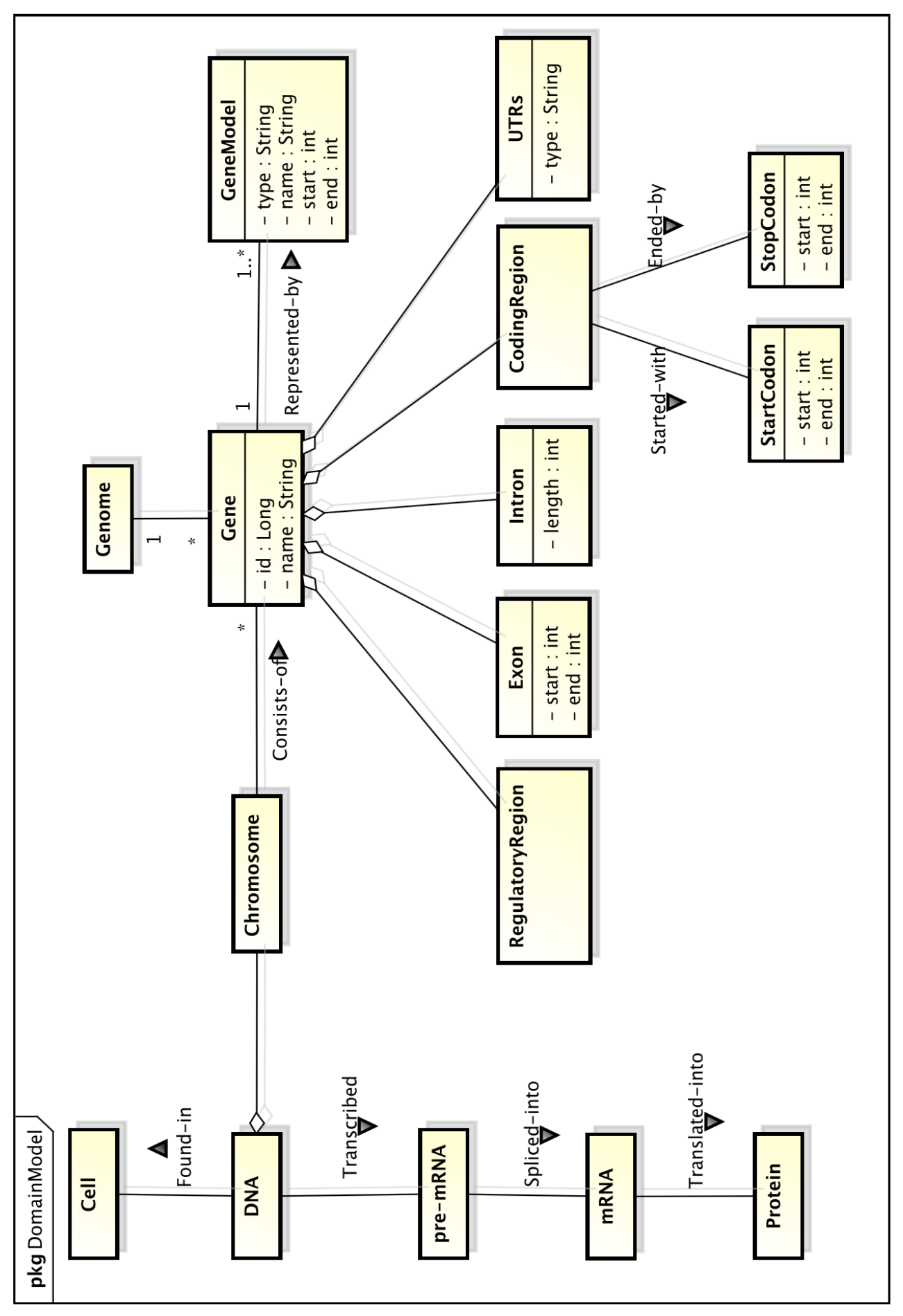

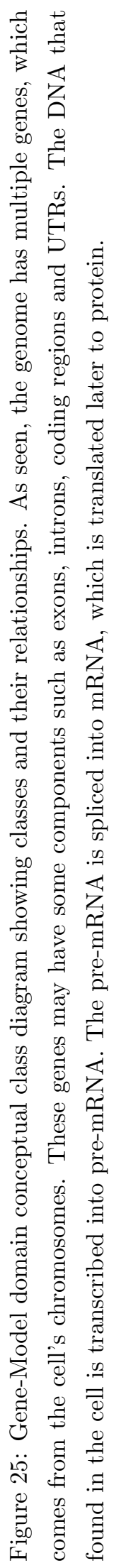




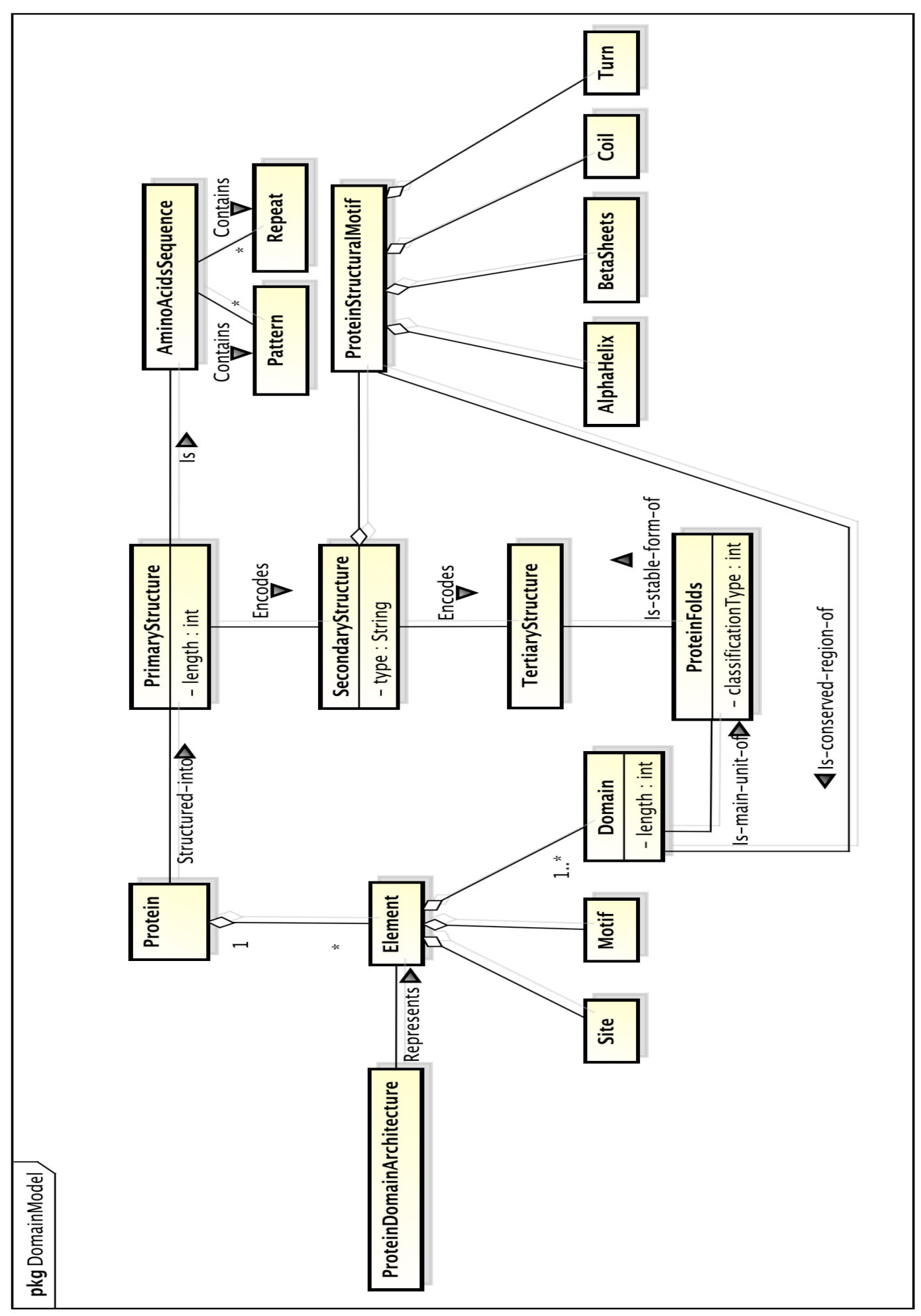

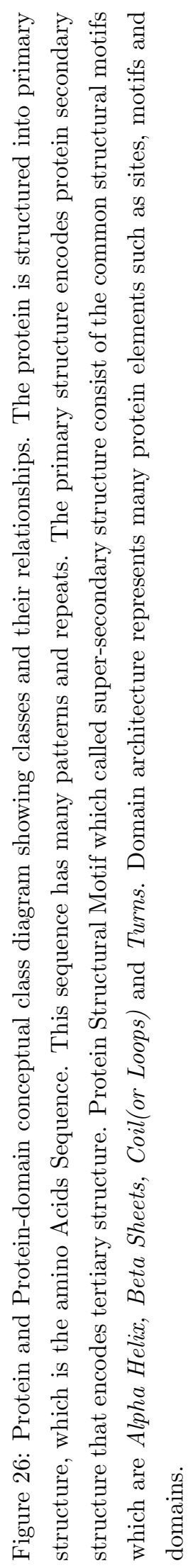




\subsection{Data Sources and Formats}

In this research, we focus on fungal genomes, so the genomic data used to produce the spreadsheets are stored in the AspGD database (www.aspgd.org). The generated data is come from standard GFF3 files, standard output files from InterProScan, aspgd file from AspGD Gene Ontology annotations file, and chromosomal feature file. For example, for A. acidus_CBS_106_47 genome the following files are processed: A_acidus_CBS_106_47_features.gff.txt created at 16-12-2013, A_acidus_CBS_106_47_iprscan.out.txt created at 19-11-2013. The other processed genomes have the same file format as A_acidus_CBS_106_47. Gene_association.aspgd.txt created at 29-6-2014 is the aspgd file that used for AspGD Gene Ontology annotations. A_fumigatus_A1163_chromosomal_feature .tab.txt created at 7-6-2014, A_nidulans_FGSC_A4_current_chromosomal_feature.tab.txt created at 7-6-2014 and A_niger_CBS_513_88_current_chromosomal_feature.tab.txt created at 6-6-2014 are the processed chromosomal feature files. All these file formats will be described later in Section 3.5.2.

\subsubsection{Data Selection}

\section{Data selection for some of protein domain architecture}

For the protein domain architecture, we did an analysis to understand what are the most repeated domains. Since there is no standard to visualize the protein domain architecture as mentioned in Chapter 2 Section 2.4.3 and CGene spreadsheets are implemented to be fixed, this study will help in case if we want to present some domains in different colors/shapes to help the user notice them. This analysis is done on five different standard output files which have protein domain predictions information that was predicted using IprScan software from the InterPro DB. The files are collected for five interested fungi according to the contents of AspGD DB [AspGD, 2014], covering the following genomes: A. nidulans, as an excellent model organism; A. fumigatus which is an important pathogen of the immunocompromised; A. flavus which is an important agriculture toxin producer; A. niger and A. oryzae which are two well known species that used in industrial processes. The analysis also includes testing 11547 distinct InterPro entries from interpro2go.txt file that found in InterPro, 2014 website. For each InterPro entry, the frequencies is counted as the sum of the entry occurrence in the five fungi. We find out that 3472 entries out of the 11547 occur at least once in the five fungi. Then, Pareto chart is applied for all the InterPro entries. First, we applied Pareto principle 80:20 rule, which states that $80 \%$ of the effects come from $20 \%$ of the causes Koch, 2011. This chart used to see what are the most important domains (or most repeated) as seen in Figure 27. However, in this figure we see that large number of entries is included in the $20 \%$. Around 666 out of 3472 entries will be selected by applying this chart. These entries are large dataset to present in different shapes/colors and they are difficult to distinguish from users, especially some of them are repeated only less than ten times. Consequently, for the sake of clarity we zoomed out the chart, and only the top 25 domains were selected as seen in Figure 28 and Table 3 In this table we picked up the top 25 repeated domain entries. This analysis helps to see what are the important domains, which is the top 25 domains from the domains that included in the $20 \%$ rule. After applying this study, these domains can be visualized in CGene spreadsheet in different shapes/colors 


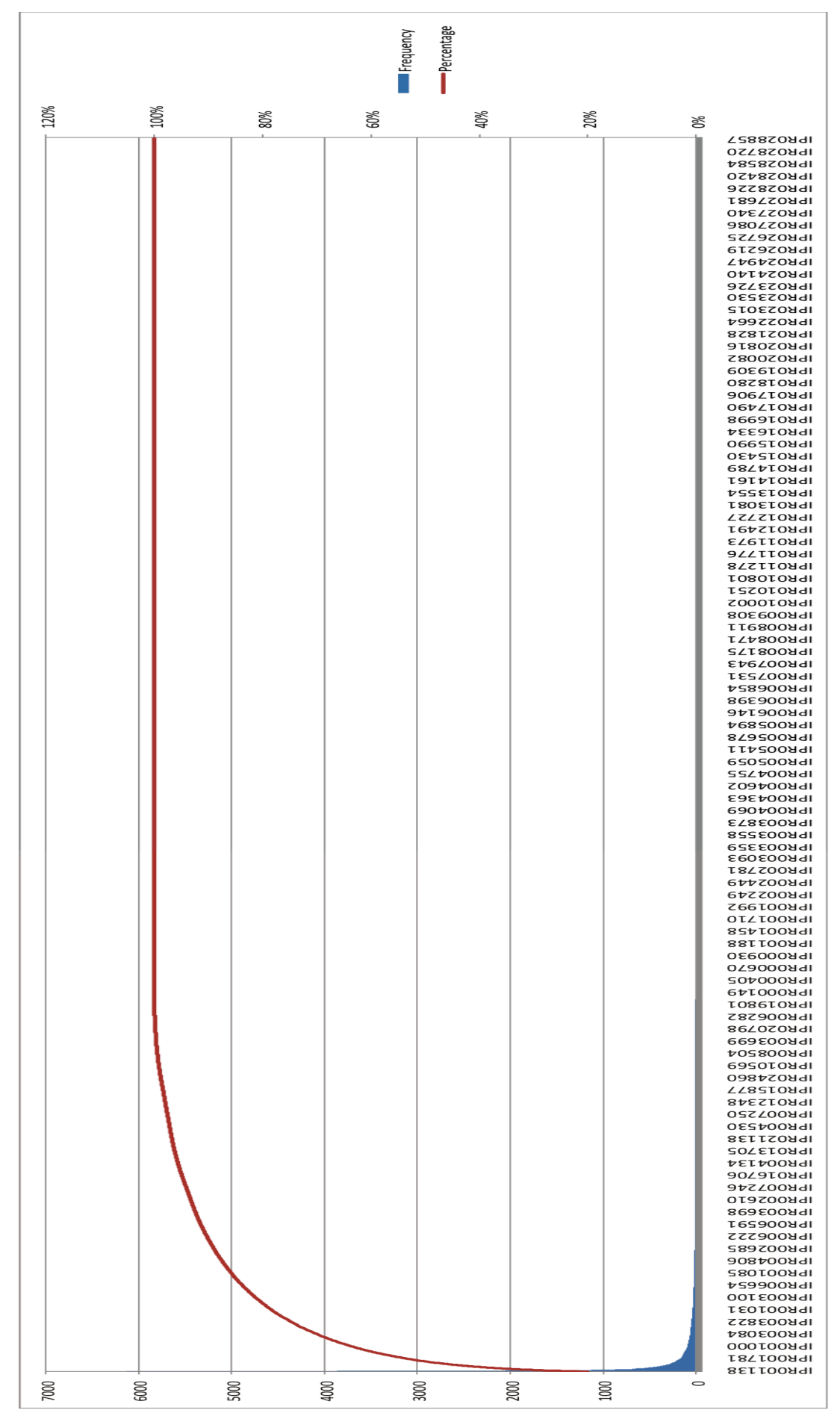

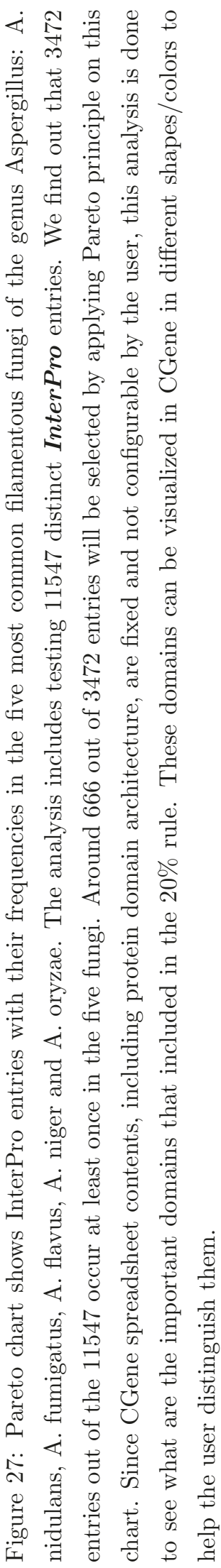




\begin{tabular}{|c|c|c|c|}
\hline InterPro Entry & Frequency & $\begin{array}{l}\text { Cumulative } \\
\text { Frequency }\end{array}$ & $\begin{array}{l}\text { Cumulative } \\
\text { Percentage }\end{array}$ \\
\hline IPR001138 & 6130 & 6130 & $4 \%$ \\
\hline IPR001680 & 5931 & 12061 & $9 \%$ \\
\hline IPR002110 & 3867 & 15928 & $11 \%$ \\
\hline IPR001128 & 3786 & 19714 & $14 \%$ \\
\hline IPR002401 & 2329 & 22043 & $16 \%$ \\
\hline IPR003663 & 2064 & 24107 & $17 \%$ \\
\hline IPR007219 & 2056 & 26163 & $19 \%$ \\
\hline IPR002198 & 1704 & 27867 & $20 \%$ \\
\hline IPR011701 & 1581 & 29448 & $21 \%$ \\
\hline IPR003042 & 1344 & 30792 & $22 \%$ \\
\hline IPR003593 & 1301 & 32093 & $23 \%$ \\
\hline IPR019734 & 1134 & 33227 & $24 \%$ \\
\hline IPR000504 & 1046 & 34273 & $25 \%$ \\
\hline IPR015943 & 992 & 35265 & $25 \%$ \\
\hline IPR011009 & 911 & 36176 & $26 \%$ \\
\hline IPR001757 & 874 & 37050 & $27 \%$ \\
\hline IPR001650 & 852 & 37902 & $27 \%$ \\
\hline IPR000719 & 832 & 38734 & $28 \%$ \\
\hline IPR017986 & 794 & 39528 & $28 \%$ \\
\hline IPR011990 & 763 & 40291 & $29 \%$ \\
\hline IPR001199 & 687 & 40978 & $29 \%$ \\
\hline IPR001806 & 682 & 41660 & $30 \%$ \\
\hline IPR002403 & 654 & 42314 & $30 \%$ \\
\hline IPR003439 & 650 & 42964 & $31 \%$ \\
\hline IPR016024 & 647 & 43611 & 31\% \\
\hline
\end{tabular}

Table 3: The top 25 selected InterPro domains data from the five most common filamentous fungi of the genus Aspergillus: A. nidulans, A. fumigatus, A. flavus, A. niger and A. oryzae. These domains are selected from the 11547 entries that showed in Figure 27. Since CGene spreadsheet contents, including protein domain architecture, are fixed and not configurable by the user, this analysis is done to see what are the top repeated domains. These domains can be visualized in CGene in different shapes/colors to help the user distinguish them. 


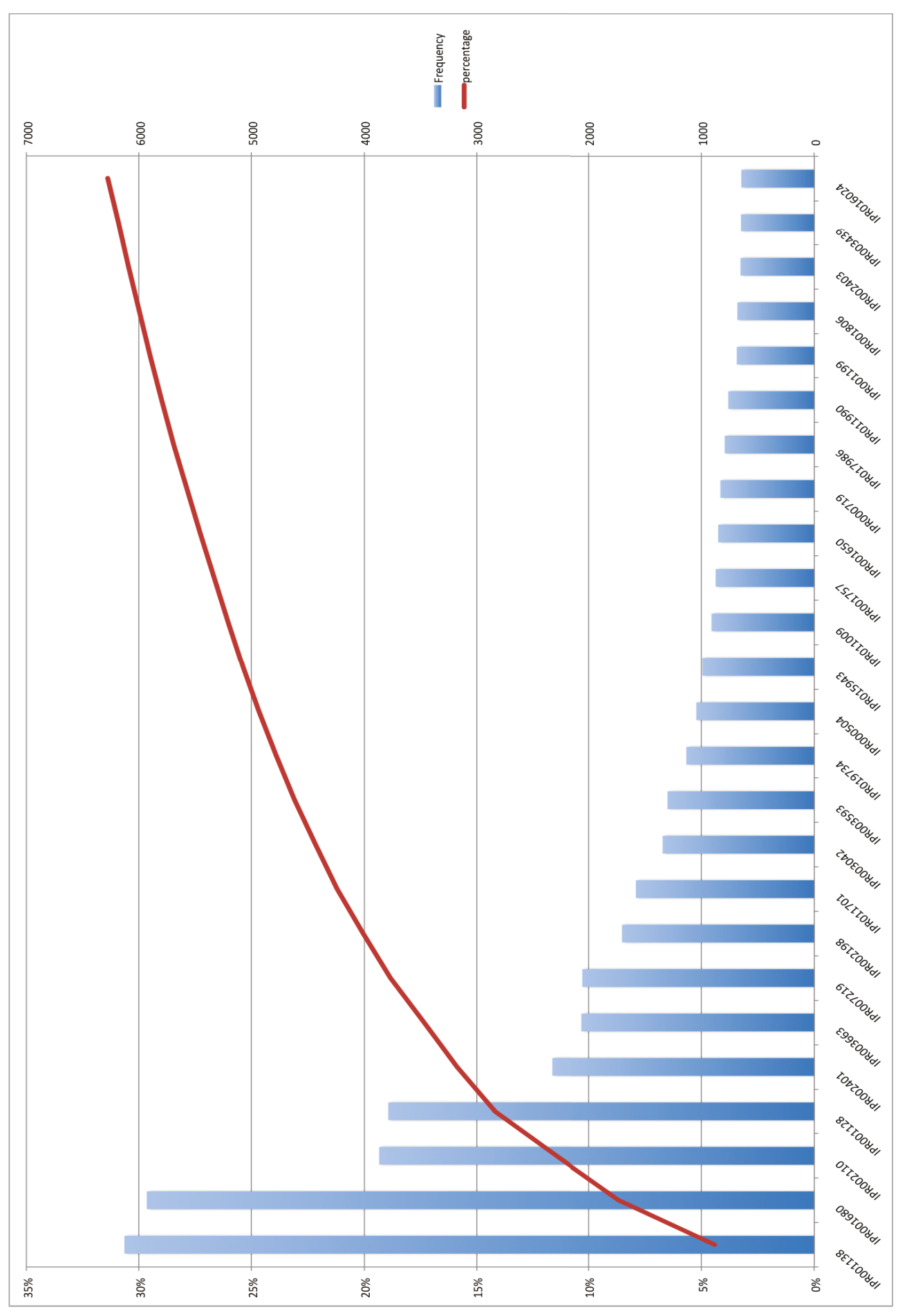

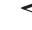

额

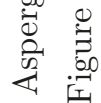

号.

D.

I

\begin{tabular}{c}
4 \\
\hline \\
50
\end{tabular}

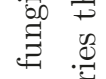

苛

릉

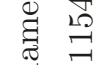

굼

궁

छี

$\begin{array}{cc}0 & 0 \\ 0 & 0 \\ 0 & 4\end{array}$

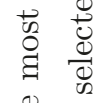

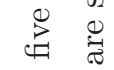

o

.

응

.

है

0

通

ज

$\stackrel{0}{0}$

离

次

요 $)$

\&

ज1

登

की

苞

兽

ค.

$\ddot{\sim}$

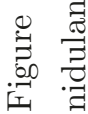




\section{Go Slims data selection for CGenome visual spreadsheet}

Go Slims, discussed previously in Chapter 2. Section 2.3.3 that used for Go Slims bitmap and Go Slims histogram in CGenome spreadsheet are Go Slims Aspergillus which selected from Gene Ontology GeneOntology, 2014 website (http://geneontology.org/page/go-slim-and-subset-gui de). In GeneOntology, 2014, slims are named to be in agreement with the application that uses these slims. For example, Go Slims Aspergillus file includes terms that mainly valuable when annotating Aspergillus. There is also a generic Go Slims file that used for most applications without species preference. Before creating CGenome spreadsheet, we did comparative analysis to have an idea about the GO Slims and to see what are the most repeated Go Slims Aspergillus in four Aspergillus fungi which are: A.nidulans_FGSC_A4, A.fumigatus_Af293, A.niger_CBS_513_88 and A.oryzae_RIB40. This analysis is done before implementing Go Slims bitmap and Go Slims histogram. By applying the analysis, we found out that protein binding GO Slim (ID: GO:0005515) has the highest number of occurrence among other molecular function Go Slims Aspergillus for all the four genomes with 4958, 3963, 3519 and 4024 for A. niger, A. nidulans, A. fumigatus and A. oryzae respectively as shown in Table 4 Figure 29 shows the same information but as percentage of occurrence instead of number of occurrence. In this figure, protein binding has the highest percentage of occurrence for all the four genomes as $46.3 \%, 43.8 \%, 44.9 \%$ and $43.0 \%$. In Table 5 nucleus GO Slim (ID: GO:0005634) has the highest number of occurrence among cellular component Go Slims Aspergillus for all the four genomes with 2609, 2333, 1696 and 1832 for A. niger, A. nidulans, A. fumigatus and A. oryzae respectively. In Figure 30, nucleus GO Slim has the highest percentage of occurrence for all the four genomes as $59.0 \%, 54.9 \%, 49.4 \%$ and $48.2 \%$. For biological process Go Slims Aspergillus, carbohydrate metabolic proces GO Slim (ID: GO:0005975) has the highest number of occurrence for all the four genomes with 875, 907, 941 and 943 as shown in Table 6 it has also the highest percentage of occurrence as $27.9 \%, 30.2 \%, 32.7 \%$ and $29.9 \%$ for A. niger, A. nidulans, A. fumigatus and A. oryzae respectively as shown in Figure 31. Some Slims did not occur at all in the four genomes such as lipase activity and site of polarized growth molecular function slims, membrane fraction cellular component slim and cellular respiration biological process slim.

After creating CGenome spreadsheet, we compare the results from the analysis and the images that showed in the spreadsheet for Go Slims bitmap and Go Slims histogram. The drawn images should have the same results that obtained from the analysis. 


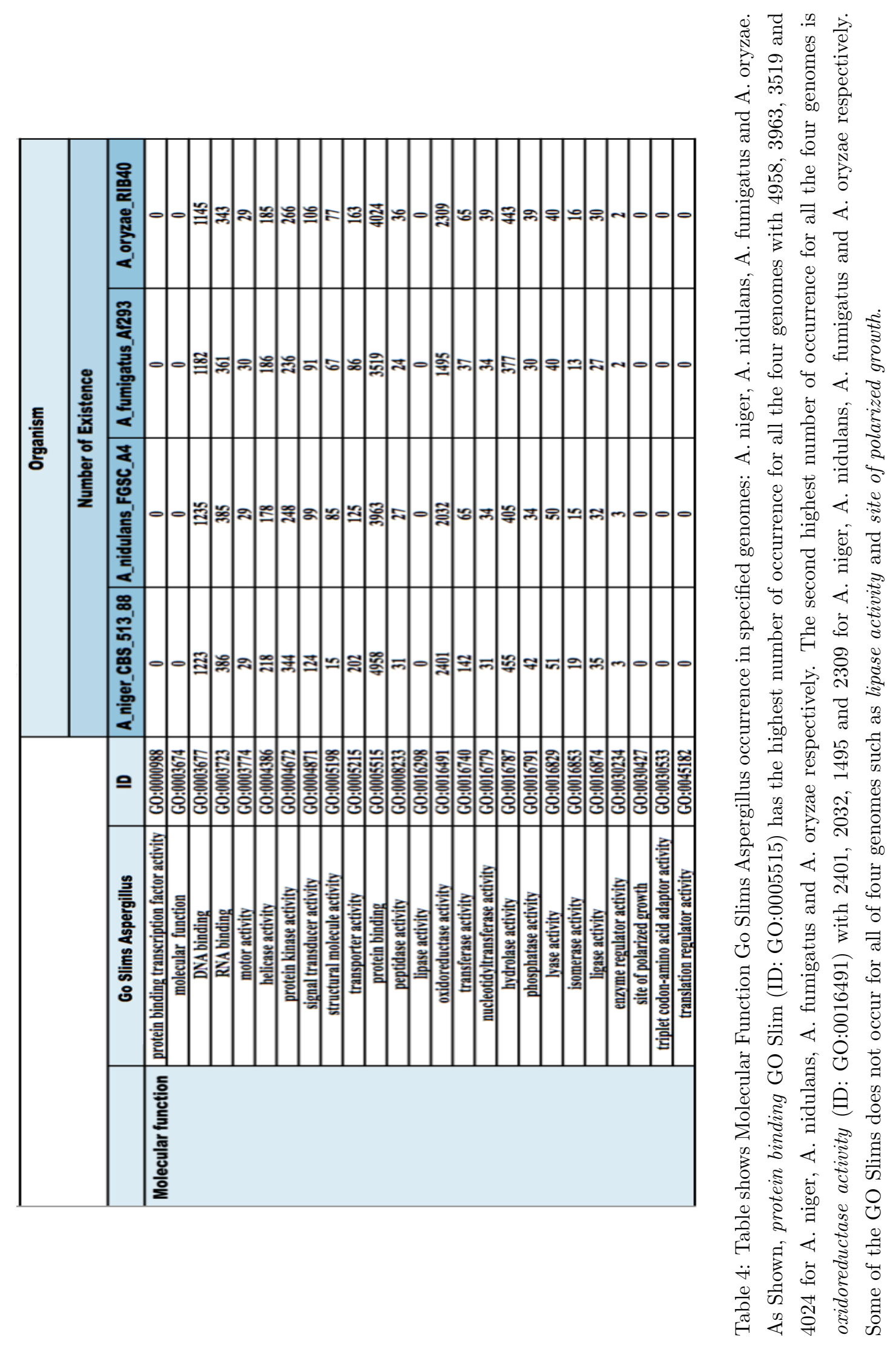




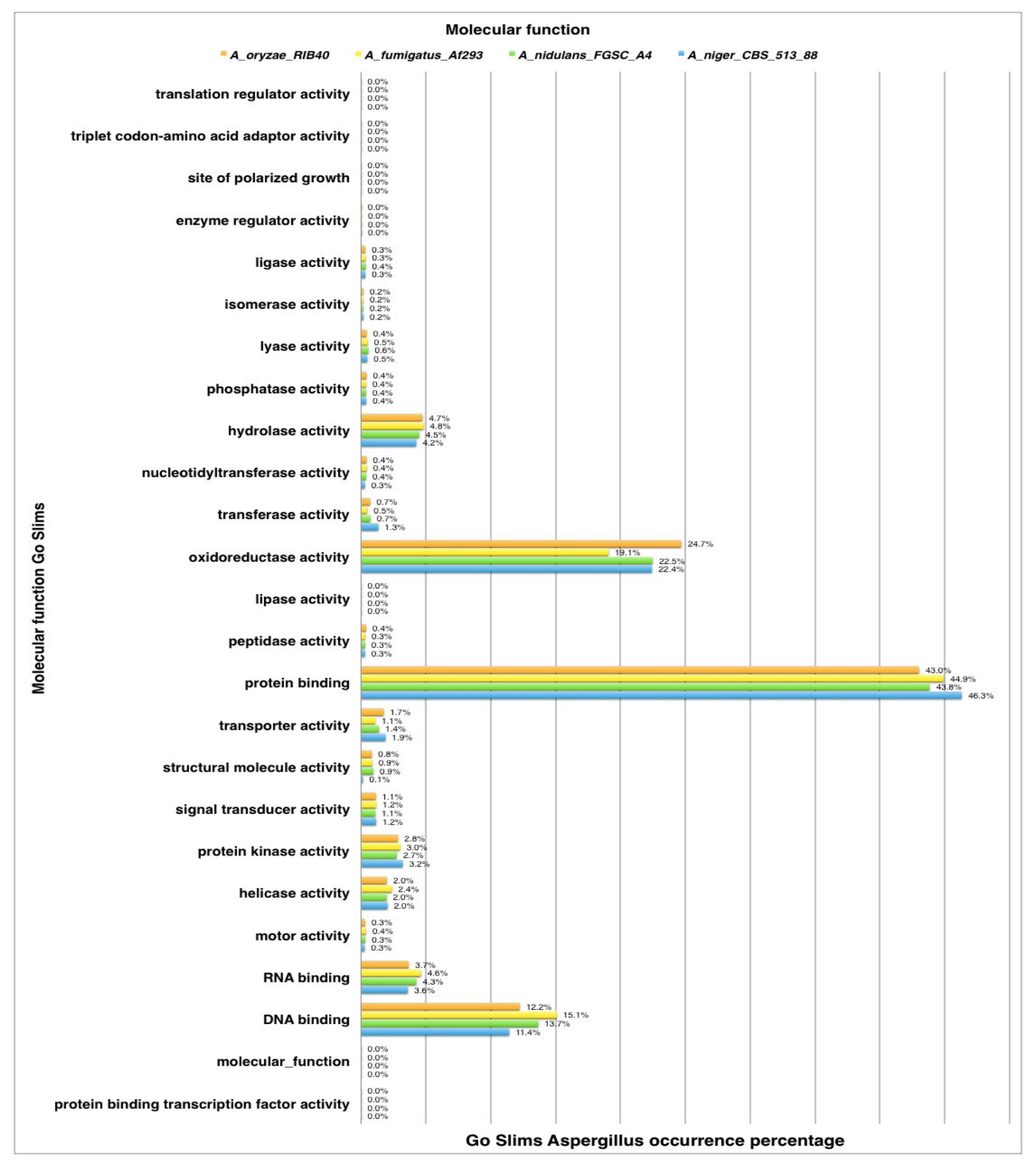

Figure 29: Chart shows Molecular Function Go Slims Aspergillus occurrence percentage in the specified genome. As Shown, protein binding GO Slim (ID: GO:0005515) has the highest percentage of occurrence for all the four genomes as $46.3 \%, 43.8 \%, 44.9 \%$ and $43.0 \%$ for A. niger, A. nidulans, A. fumigatus and A. oryzae respectively. The second highest percentage of occurrence for all the four genomes is oxidoreductase activity (ID: GO:0016491) with $22.4 \%, 22.5 \%, 19.1 \%$ and $24.7 \%$ for A. niger, A. nidulans, A. fumigatus and A. oryzae respectively. Some of the GO Slims has zero occurrence percentage for all of four genomes such as lipase activity and site of polarized growth. 


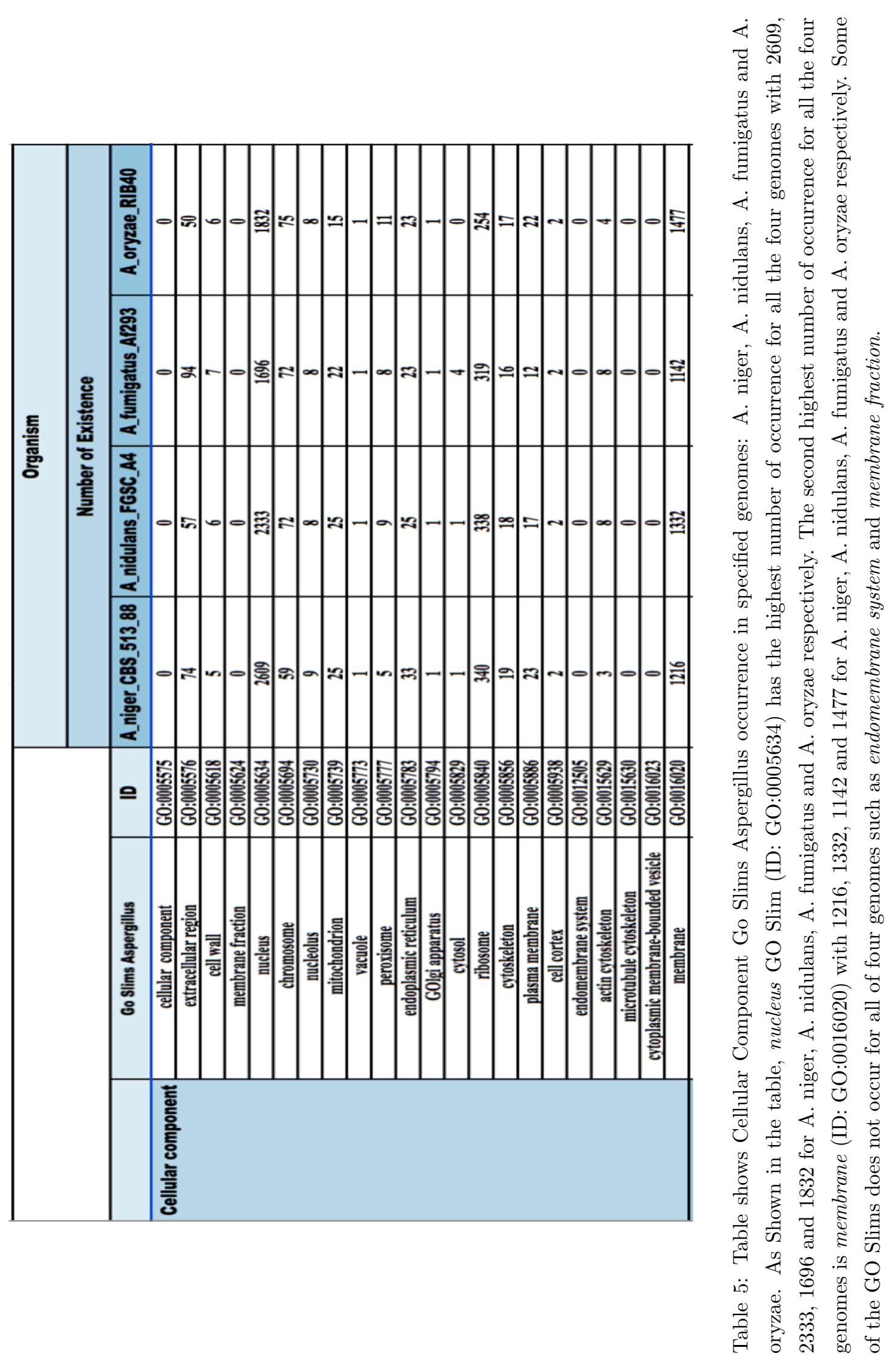




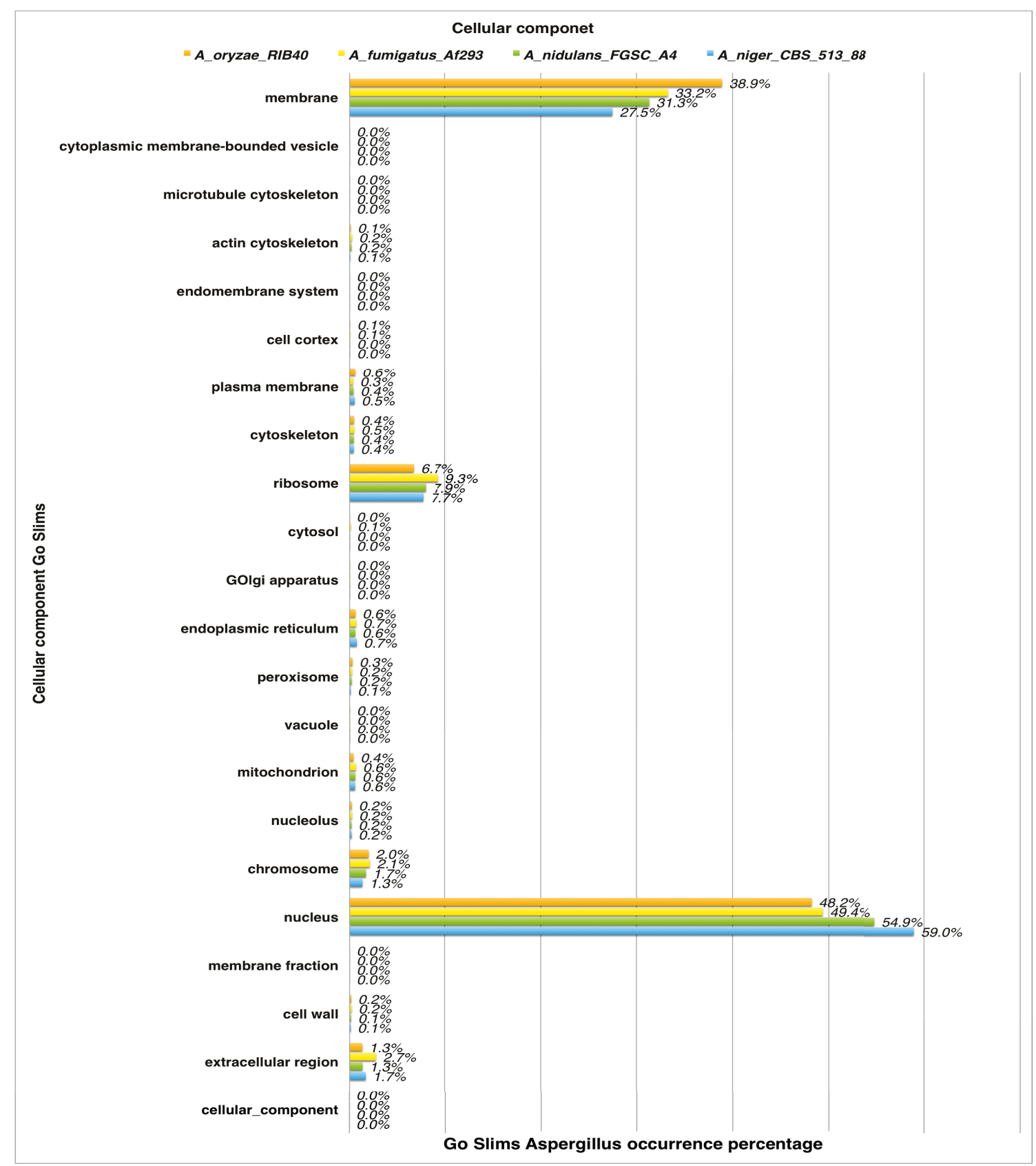

Figure 30: Chart shows Cellular Component Go Slims Aspergillus occurrence percentage in the specified genome. As Shown, nucleus GO Slim (ID: GO:0005634) has the highest percentage of occurrence for all the four genomes as $59.0 \%, 54.9 \%, 49.4 \%$ and $48.2 \%$ for A. niger, A. nidulans, A. fumigatus and A. oryzae respectively. The second highest percentage of occurrence for all the four genomes is membrane (ID: GO:0016020) with $27.5 \%, 31.3 \%, 33.2 \%$ and $38.9 \%$ for A. niger, A. nidulans, A. fumigatus and A. oryzae respectively. Some of the GO Slims has zero occurrence percentage for all of four genomes such as endomembrane system and membrane fraction. 


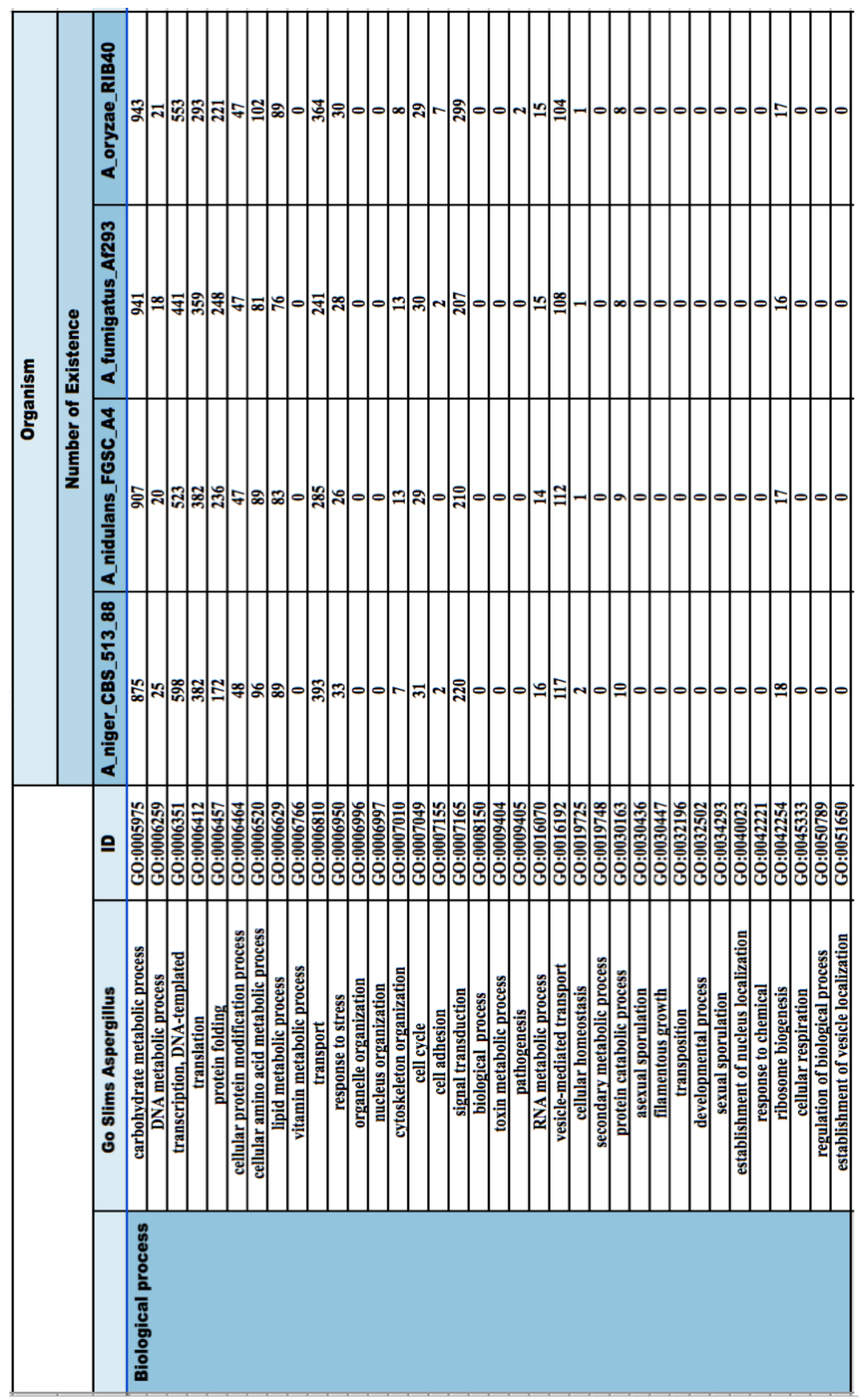

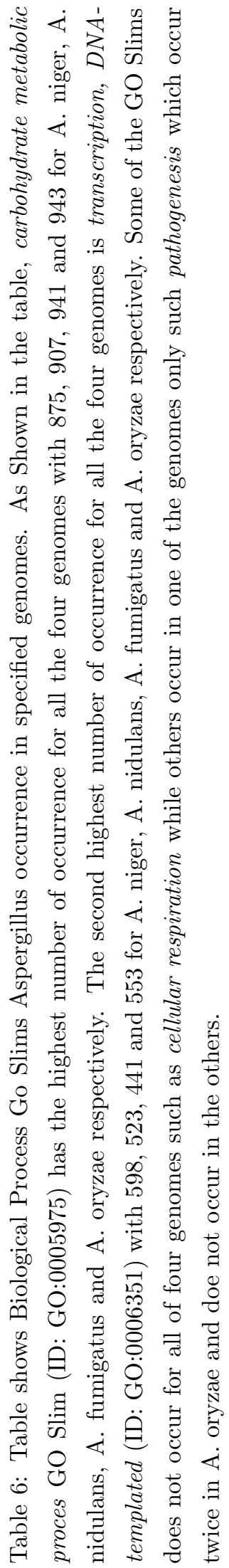




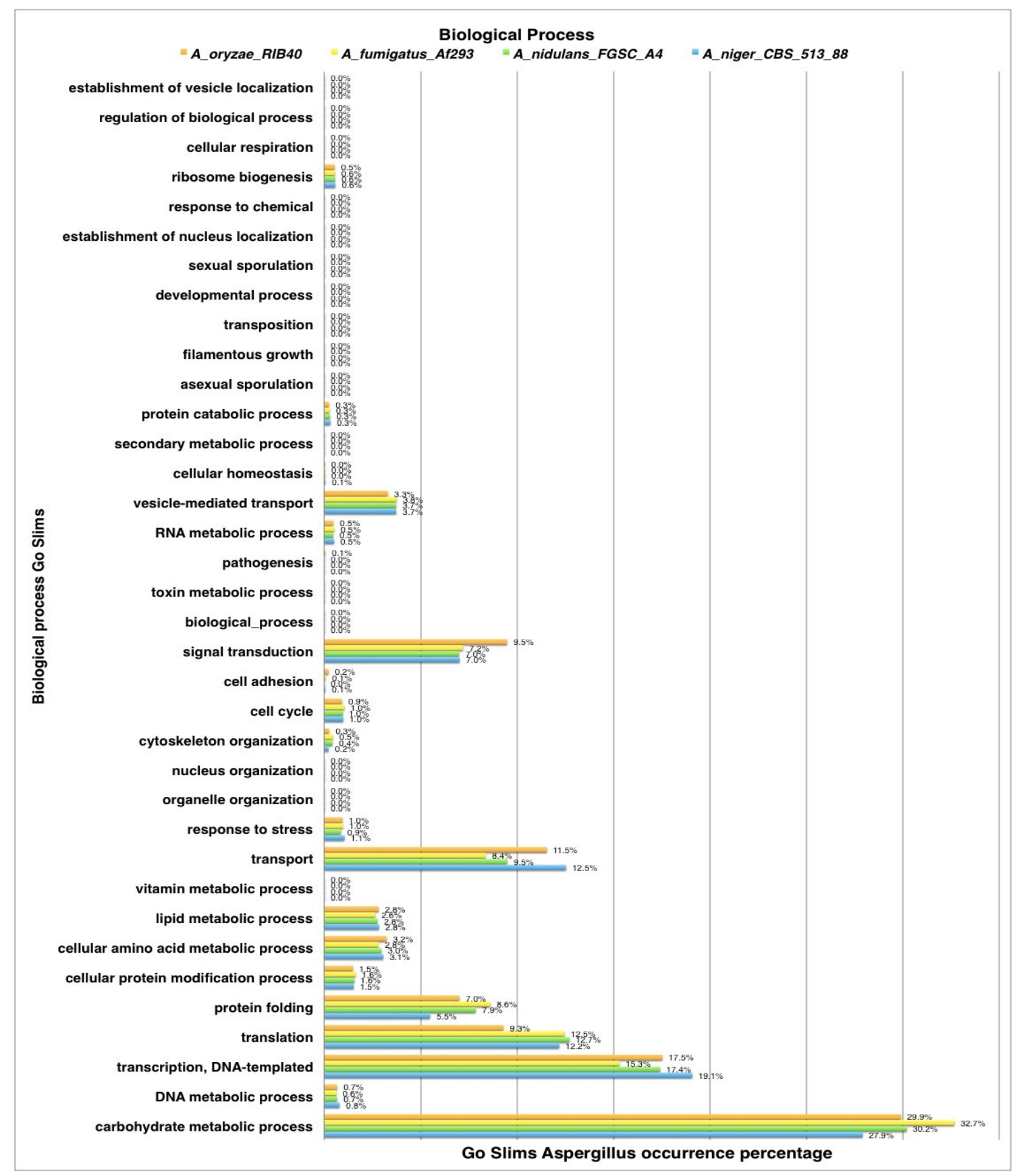

Figure 31: Chart shows Biological Process Go Slims Aspergillus occurrence percentage in the specified genome. As Shown, carbohydrate metabolic proces GO Slim (ID: GO:0005975) has the highest percentage of occurrence for all the four genomes as $27.9 \%, 30.2 \%, 32.7 \%$ and $29.9 \%$ for A. niger, A. nidulans, A. fumigatus and A. oryzae respectively. The second highest percentage of occurrence for all the four genomes is transcription, DNA-templated (ID: GO:0006351) with 19.1\%, 17.4\%, 15.3\% and $17.5 \%$ for A. niger, A. nidulans, A. fumigatus and A. oryzae respectively. Some of the GO Slims has zero occurrence percentage for all of four genomes such as cellular respiration. 


\subsubsection{Data Formats}

As mentioned in Section 3.5, there are several data files that used in CGene and CGenome HTML5 visual spreadsheet system. These files are: Generic Feature Format (GFF) files, standard output files from InterProScan, Aspgd file for AspGD Gene Ontology Annotations file, chromosomal feature file, Go Slims file and Interpro2go flat file. Some of these files are dwonloaded from Aspergillus website while others from different websites such as InterPro website.

In Aspergillus genome website (www.aspgd.org), there is download directory which has many sub-directories, where the data can be downloaded. The usded sub-directories are: brows download/gff/, brows download/domains/, brows download/chromosomal_feature_files/ and brows download/go/.

\section{Generic Feature Format (GFF) file}

"GFF is a format for describing genes and other features associated with DNA, RNA and Protein sequences Sanger-Institute, 2014."

Gff files are found in download/brows download/gff sub-directory. This directory contains files that store data about several Aspergillus species in the Generic Feature Format (GFF). Each GFF3 file has features description, which includes chromosomes, ORFs, CDSs, introns, sequence gaps, intergenic regions, etc. These files are parsed to extract the information about each gene and its features such as axons, gene ID, and gene name. The introns and the gene length are calculated from the available data. For more details about the Generic Feature Format (GFF) specification, please visit the URLs: (http://www.sequenceontology.org/gff3.shtml) and (http://www.sang er.ac.uk/resources/software/gff/spec.html.

\section{Standard output files from InterProScan}

These files are found in download/brows download/domains/ directory. This directory has Protein Domain Predictions information that was predicted using IprScan software from the InterPro DB. Each file has InterPro domains information that related to Aspergillus genome such as systematic identifier of the input sequence, length of the sequence, analysis method, etc. All these informations are organized in tab-delimited file which each column represent feature. For more details about the InterPro database and IprScan output file, please visit the URL: ftp://ftp.ebi.ac.uk/pub/soft ware/unix/iprscan/README.html).

\section{Aspgd file for AspGD Gene Ontology Annotations}

This file is found in download/brows download/go/ directory. This directory consists of file that has all Gene Ontology (GO) annotations for AspGD genes. The file is tab-delimited file and has GO annotations related to Aspergillus genome. This file follows the standard file format of GO Consortium for gene_association files GeneOntology, 2014. For more details, please visit the

URLs: (http://geneontology.org/) and (http://www.aspergillusgenome.org/download/go/ gene_association_README.txt). 


\section{Chromosomal feature file}

These files are found in brows download/chromosomal_feature_files/ directory. This directory consists of data for several Aspergillus species in chromosomal_feature.tab file format. Each file about one Aspergillus species, and has information about chromosomal features in AspGD such as feature name, aliases (synonyms), descriptions, type, chromosomal coordinates, etc. All this information is organized in tab-delimited file which each column represent feature. For more details, please visit the URL: (http://www.aspergillusgenome.org/download/chromosomal_feature_files/).

\section{Go Slims file}

Go Slims that used for Go Slims bitmap and Go Slims histogram in CGenome spreadsheet are Aspergillus Slims which selected from GeneOntology, 2014 website (http://geneontology.org /page/go-slim-and-subset-guide). Aspergillus slim includes terms that mainly beneficial when annotating Aspergillus while the generic slim is used for most applications without species preference.

\section{Interpro2go flat file}

This file has a list of Gene Ontology (GO) terms, which are mapped to InterPro entries from InterPro website. Interpro2go file is used in this research to count the InterPro domain appearance in the Protein Domain Predictions file based on the GO terms. To downloads, see: (http://www.ebi.ac .uk/interpro/download.html).

\subsection{Data File Preprocessing}

In order to achieve the wanted functionality from CGene and CGenome, the gathered files from AspGD DB (http://www . aspergillusgenome.org) should be analyzed. Below is general description of the analysis that done on the files.

\section{- Code: GFFParser.java}

Input: .gff file that contains features of the genome assembly in Generic Feature Format (GFF) from AspGD DB (http://www.aspergillusgenome.org for a specific organism.

Output: .txt file for the entered organism called OrganismNameGFFExtracted.txt.

Functionality: extract some of the features from the GFF file, then create a new file which has the desired features for the system from the GFF file. 


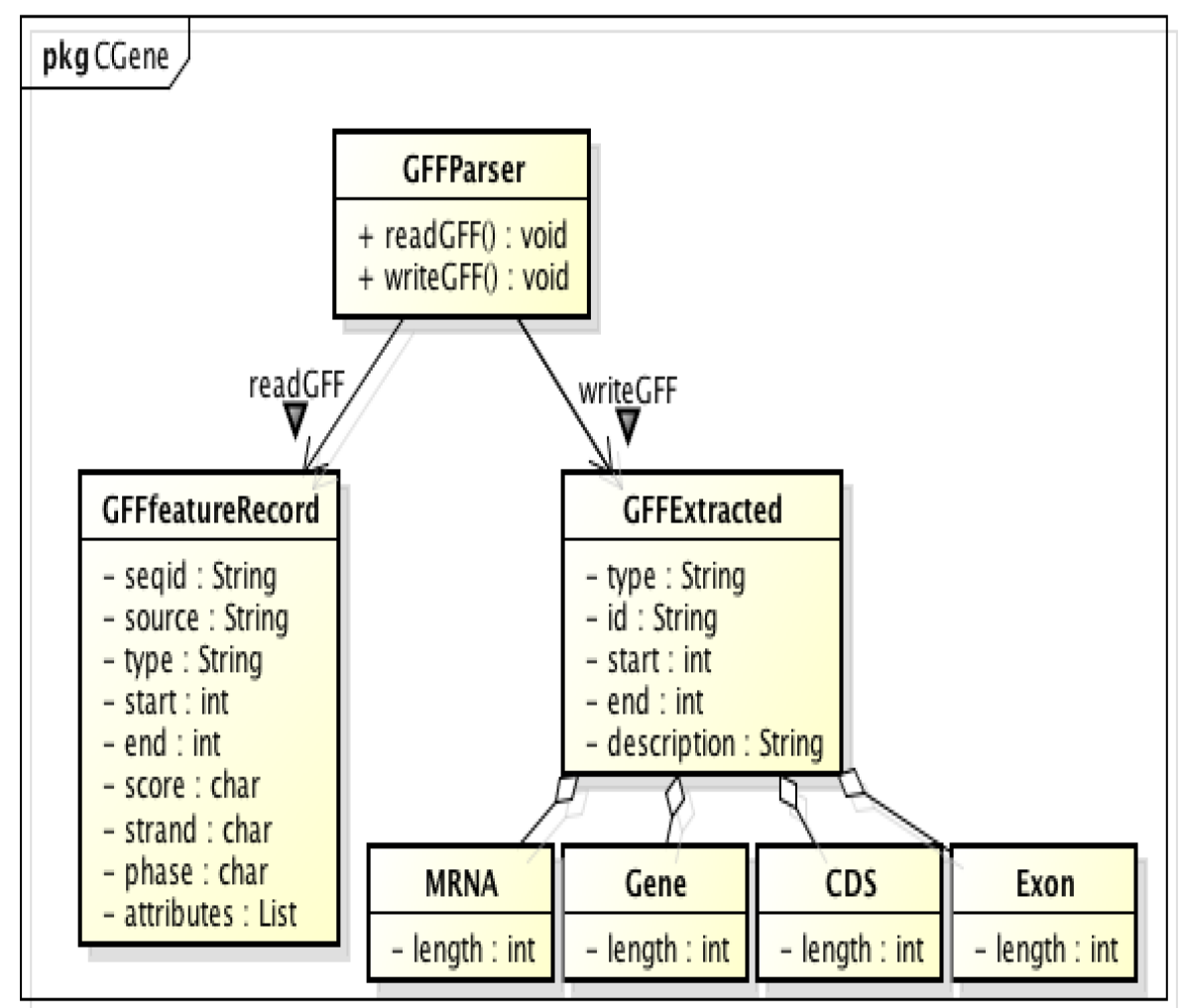

Figure 32: GFFParser.java code UML diagram.

- Code: ProteinDomainParser.java

\section{Input:}

- .out file from domains directory in AspGD DB (http://www.aspergillusgenome.org) for a specific organism.

Output: .txt file for the entered organism.

- OrganismNameExtractedIprScanOutputFile.txt

Functionality: extract the desired features from IprScan Output File and create new .txt file which has the extracted features from the domain file. 


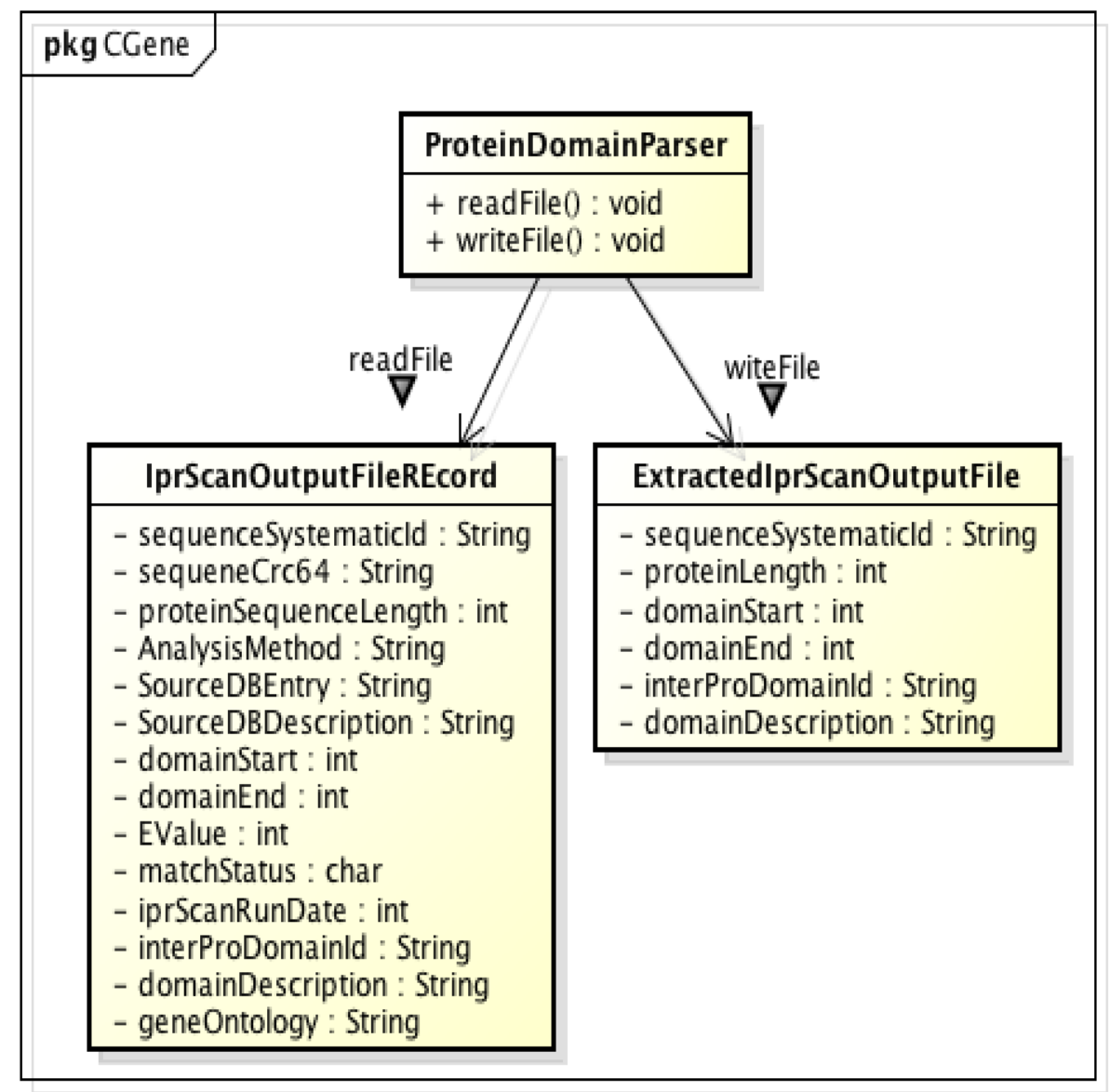

Figure 33: ProteinDomainParser.java code UML diagram.

\section{- Code: GeneProteinLinking.java}

Input: the two resulted .txt files from ProteinDomainParser.java and GFFParser.java for a specific organism.

1. OrganismNameExtractedIprScanOutputFile.txt

2. OrganismNameGFFExtracted.txt

Output: FeaturesLinkedFile.txt

Functionality: join the tow files by linking each gene in the (GFFExtracted.txt) file with its associated protein domains in the (ExtractedIprScanOutputFile.txt) domain file if the linked protein is available. Then place them in a single (FeaturesLinkedFile.txt) file. 


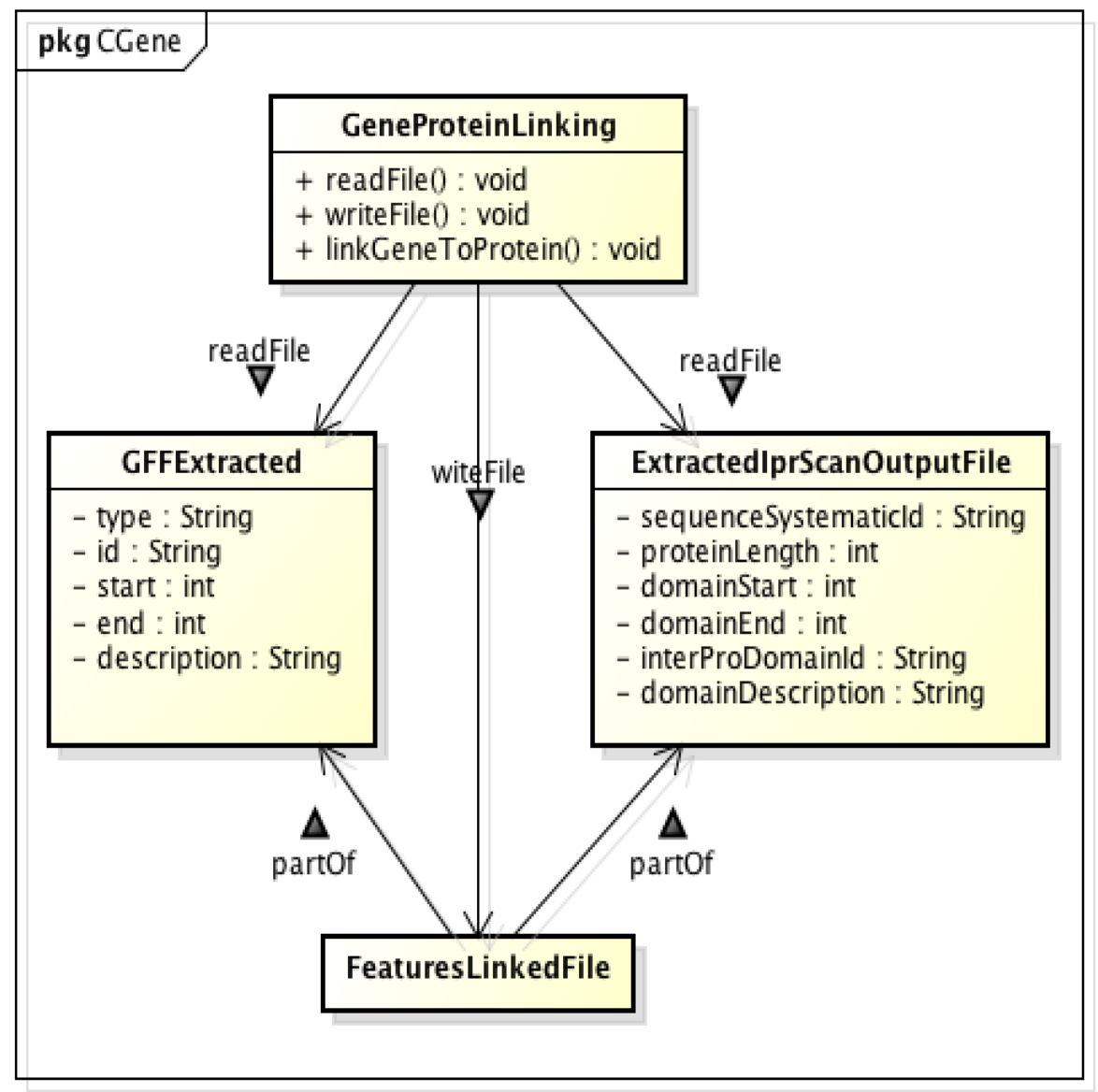

Figure 34: GeneProteinLinking.java code UML diagram.

- Code: GeneModelSVG.java

Input: (OrganismNameGFFExtracted.txt) file that created by GFFParser.java for a specific organism.

Output: multiple gene model SVG files.

Functionality: gene model SVG file is created for each gene in the entered organism.

The naming schema is:

- For gene model SVG: OrganismName_GeneID_SVGFile.svg 


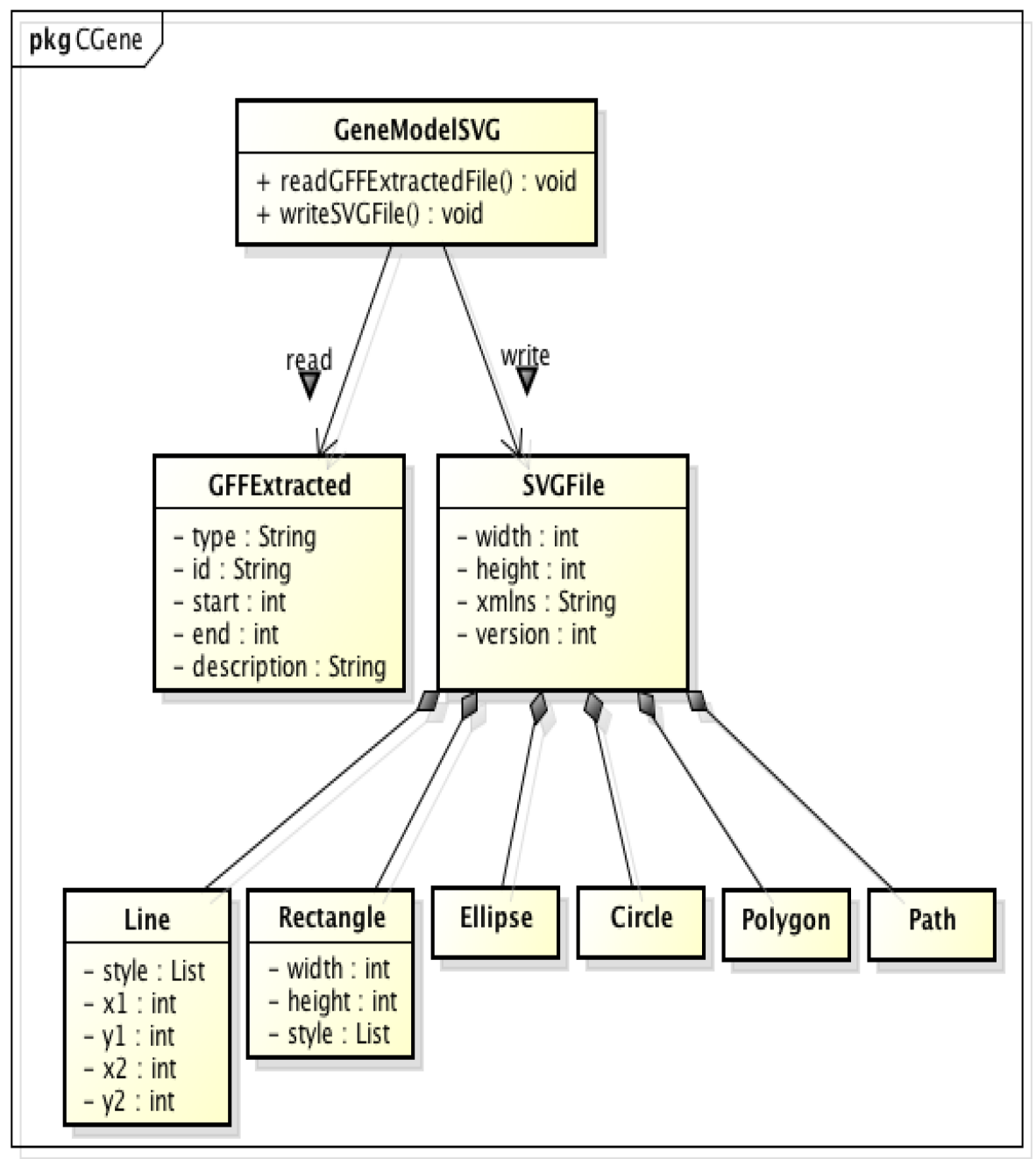

Figure 35: GeneModelSVG.java code UML diagram.

- Code: DomainArchitectureSVG.java

Input: ExtractedIprScanOutputFile.txt file that created by ProteinDomainParser.java for a specific organism.

Output: multiple protein domains SVG files.

Functionality: protein domain architecture SVG file is created for each protein.

The naming schema is:

- For protein SVG: OrganismName_GeneID_Dom_SVGFile.svg 


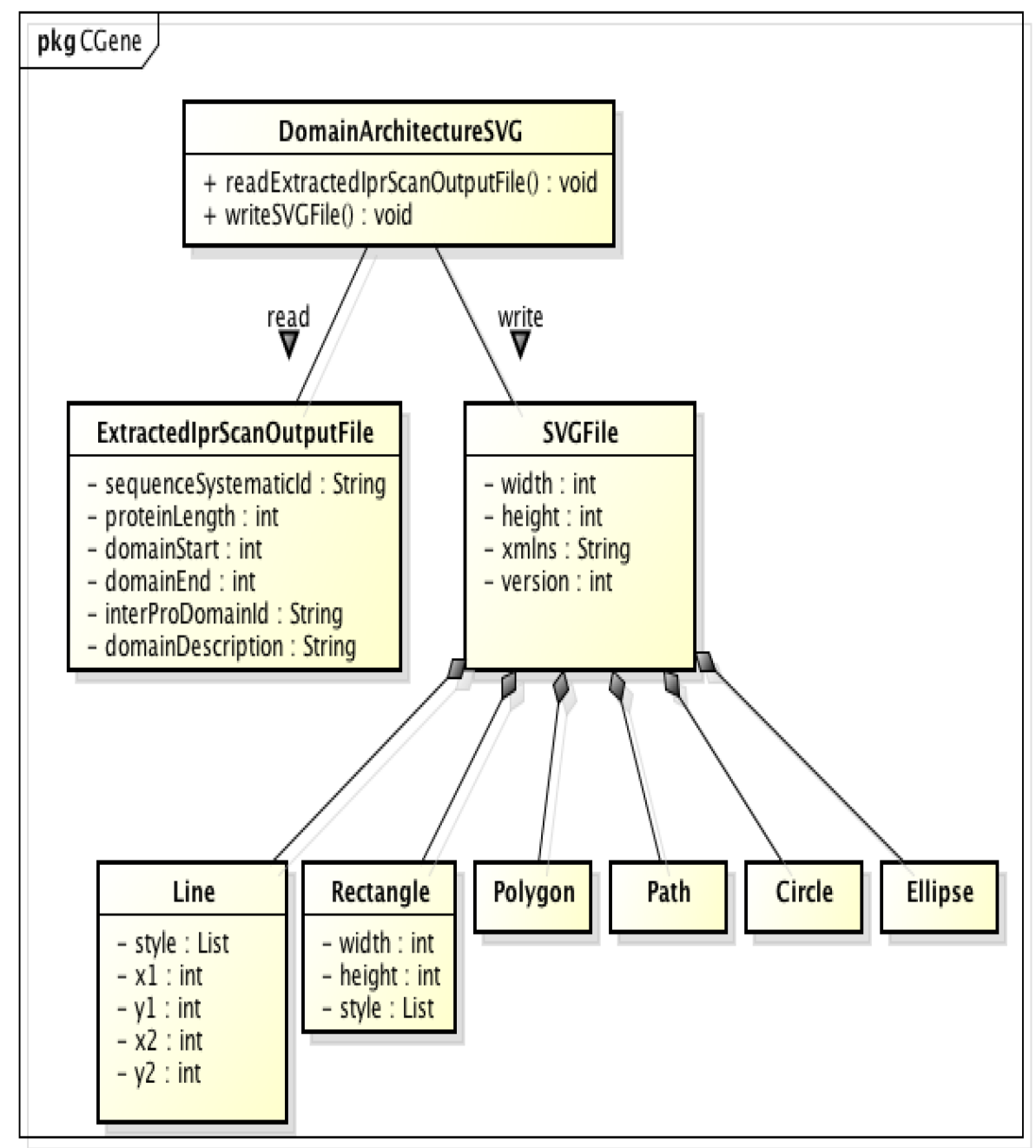

Figure 36: DomainArchitectureSVG.java code UML diagram.

- Code: ChromosomeLocationParser.java

Input: .txt file that contains chromosome features for a specific organism from AspGD DB (http: //www.aspergillusgenome.org. Output:.txt file for the entered organism.

- OrganismNameChromosomeLocationExtracted.txt

Functionality: extract some of the features from the file, then create a new file which has the desired features for Genome system. 


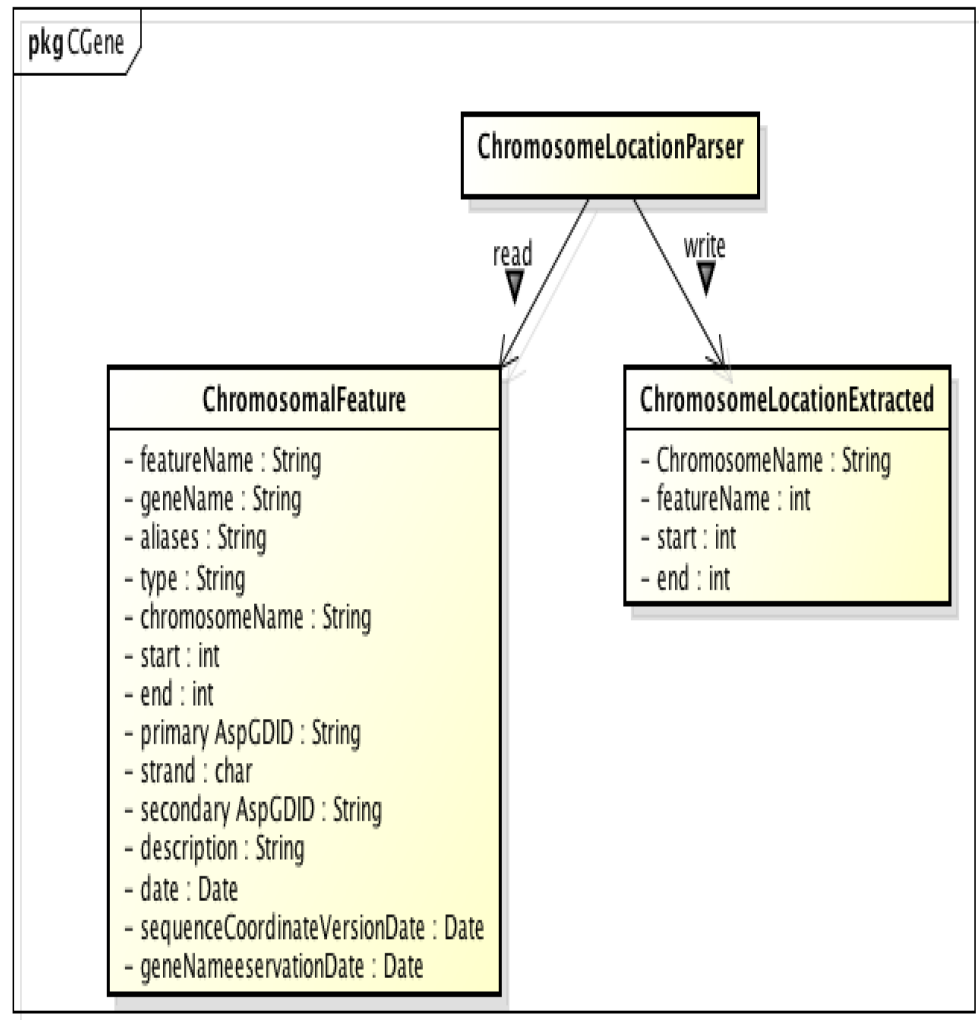

Figure 37: ChromosomeLocationParser.java code UML diagram.

- Code: CreatHTMLtable.java

Input: FeaturesLinkedFile.txt file that created by GeneProteinLinking.java for a specific organism. Output: OrganismName_HTMLTable.txt

Functionality: create the whole HTML5 table syntax for the entered organism.

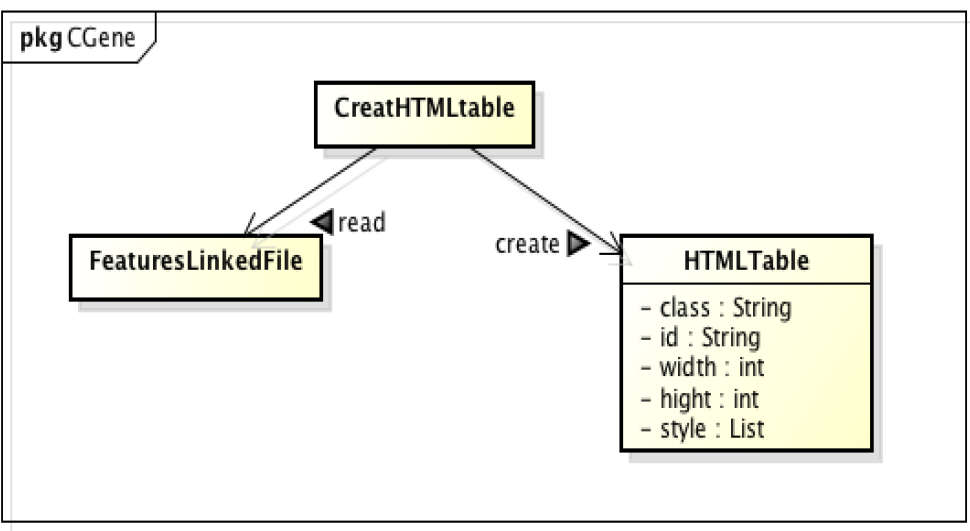

Figure 38: CreatHTMLtable.java code UML diagram. 


\subsection{Why HTML5 and SVG?}

\subsubsection{HTML5}

HyperText Markup Language v.5 (HTML5) is a modern markup language that used to create web pages, but HTML roots go back to the early 90 's. There are several versions appeared before HTML5, and the latest one was HTML 4.01. From v.4.01, the language has been improved significantly to support the latest multimedia. In 2004, Web Hypertext Application Technology Working Group (WHATWG) started to develop the specification for HTML5 and they began to add some extra features to support the web applications. Two years later, World Wide Web Consortium (W3C) cooperated with WHATWG, and their core aim was to propose a substitution for HTML 4, XHTML, and the HTML DOM Level 2. They published together the first working draft for the new version in 2008 HTML5, 2014.

HTML5 developers aimed to create a strong language that satisfies four basic principles, which are: compatibility, utility, interoperability and universal access [Pilgrim, 2010]. The first thing that they care about while adding new features is keeping everything move smoothly and compatible with the available content. Even thought new standards were added, they were developed in a way that close to which the user adapted with previously to make it easy to switch to the new HTML5 elements. For example, several of webpages analyzed by Google, and most of the web designers used to declare a DIV element with $i d=$ "header" each time they want to create a header content. In HTML5, the redundancy issue is solved, and separate $<$ header $>\ldots</$ header $>$ element is added to make things much simpler.

In addition to compatibility, HTML5 developer put the user as their main priority over the other parties such as the browsers or the specifiers themselves. Their desire was to produce a simple and

useful language as much as they could. Some of the previous features are supported such as using the CSS to spread the idea of separation between the code content and the web presentation. Using feature like CSS will increase the simplicity and reduce the redundancy, which may cause slower loading pages and hardness in code reading. Moreover, HTML5 designed to be accessed universally. Consequently, its functionality should be device and platform independent. HTML5 offers the users the ability to create dynamic, rich and interactive experiences for any platform and device with a web browser. Thats why many famous companies use HTML5 such as Netflix in for its User Experiences on Devices. It also supports most of the world languages. For this matter, Ruby annotations that used in East Asian typography is supported in a new < rubey> element Pilgrim, 2010.

In addition to the previous features, reducing the need for multimedia external plugins is one of the strongest features that have been supported strongly in HTML5. External plugins like Flash have many problems since many of them cannot be installed, may be blocked or disabled by some devices and browsers. Plugins also are difficult to integrate due to their boundaries, clipping and transparency problems.

To minimize the need for the plugins, new elements were added to perform new functionalities that not supported before HTML5. These elements such as Scalable Vector Graphics(SVG) element can be styled using CSS and scripted using JavaScript. Although SVG was available previously 
as a web plugin, its first introduction as HTML5 element was in W3C recommendation in 2001. Another example for reducing the need for multimedia plugins is watching a video on the webpage. In HTML5, a video frame can be added in a <canvas > element, and the user just click in this frame and play it. This functionality can be done in the native code without using the plugins to play the video as supposed previously Pilgrim, 2010.

Why HTML5? In addition to: simplicity, compatibility, the universal access and the other stated features, HTML5 supports many JavaScript libraries perfectly. These libraries help biological visualization in different ways such as D3.js which displays dynamic graphical forms in the web browsers. Moreover, it supports Scalable Vector Graphics (SVG) natively which is started to be used widely in biological visualization. More details about JavaScript and SVG will be stated in Section 3.7 .2 and in Section 3.7.3.

Before concluding this section, there is a some of key features that are supported in HTML5 (http: //www.w3schools.com). This features include Supporting graphics and media such as Scalable Vector Graphics(SVG), canvas (2D and 3D), audio and video. it also supports WebSocket API and protocol, indexed database and web storage, forms, micro-data, MathML, drag and drop.

\subsubsection{SVG}

Scalable Vector Graphics (SVG) is a vector image format that used to describe two-dimensional graphics, particularly it is used for displaying images in the Web browsers. It is based on Extensible Markup Language (XML) rules for encoding documents. The first full specification as HTML5 element was initiated in W3C recommendation in 2001. Before HTML5, SVG can be embedded in $<\mathrm{img}>$ element or as a link to.$S V G$ document in order to show the SVG image in the webpage. Now, it is part of HTML5 elements with $<\operatorname{svg}>\ldots .</ \mathrm{svg}>$ tags at the beginning and the end respectively.

As the name of SVG indicates, it is a vector graphic, which is distinctive from other formats such as JPEG, PNG and GIF that are raster graphics. In order to understand the importance of the Scalable Vector Graphics in web graphics, we need to differentiate between the vector graphics and one of most common image graphics format, which is raster images.

Raster vs. Vector A raster image, or bitmap, is made up of rectangular pixels that represented in a grid of $\mathrm{x}$ and $\mathrm{y}$ coordinates. All these coordinates have the same size with the same or different colors. Pixels colors are represented in the screen with a numerical value such as $R G B$, which consists of three numbers to calculate its color. The amount of the numbers that needed to calculate the colour is different depending on the type of image such as eight bits for gif image. The Canvas 2D API, one of HTML5 elements, is an example of raster graphics. Since the raster images consist of fixed number of pixels, it cannot be scaled up. Otherwise, the edges will

look pixelated and not clear as seen in Figure 39, which lost some of its details. In order to minimize this problem, the resolution should be increased which increase the size of the file consequently. 


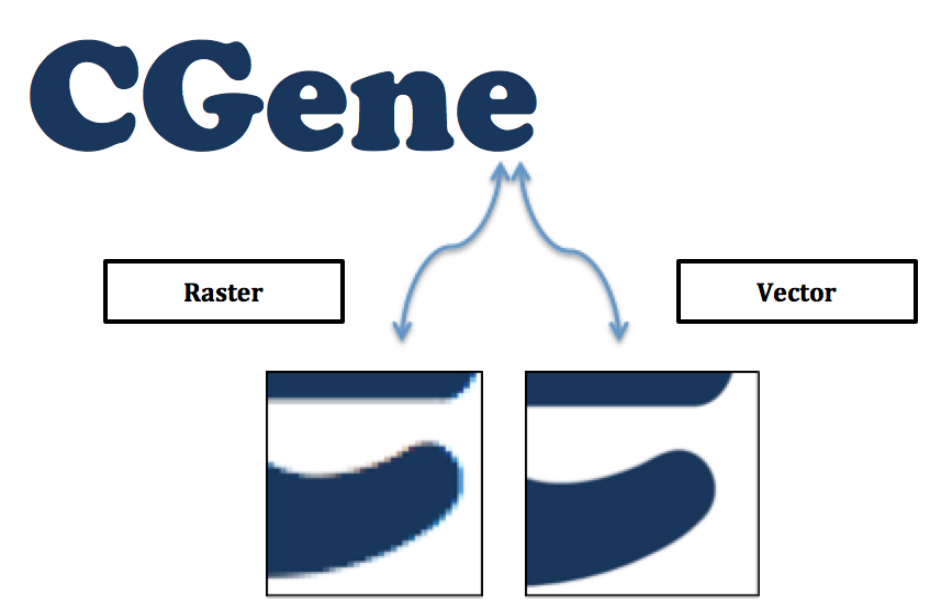

Figure 39: Illustration of the effect of magnification on vector graphics versus raster graphics. The vector-based is at the right and the raster-based at the left.(Credit: the conversion from bitmap to SVG is done in http://vectormagic.com/home website)

On the other hand, vector graphics are represented with mathematical descriptions of geometry. The images basically consist of sufficient vector points that connected by lines then filled with different colours to create diversity of closed shapes. Each point has information that guide the computer how to connect this point with other points in a straight or curved lines as seen in Figure 40. This feature makes the editing of the vector images much easier then raster one. To resize the vector images, the vector points distribute to fit the new size, and the computer just reconnect the image lines and fill the colour again.
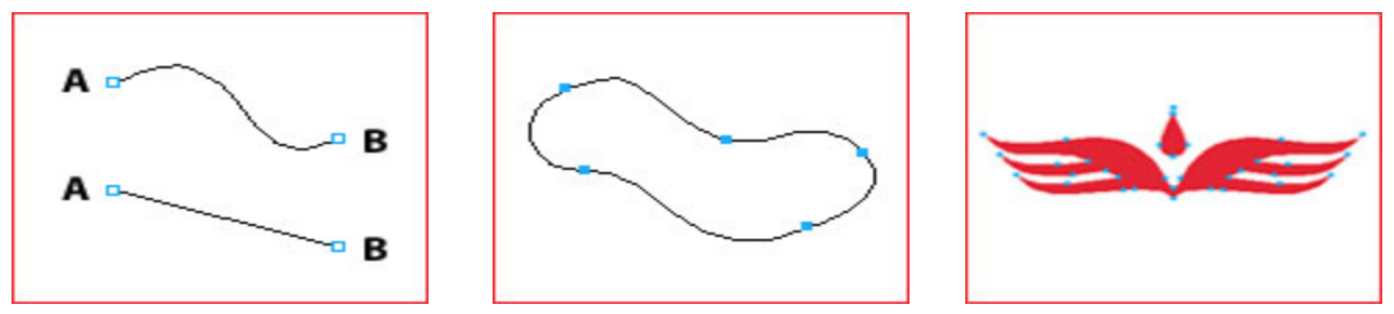

Figure 40: Vector graphics concept illustration. The left image shows two points A and B that are linked in different ways deepening on the descriptive information in the SVG file. The middle image shows five points connected together to create the shape. The right one shows enough points to make a specific image.(Credit: these images are taken from http://freerangestock.com/underst anding/vector_bitmap/Part2_Vector.html website)

\subsubsection{Basic Vector Shapes}

SVG has six basic shape elements: <line $>,<$ circle $>$, <ellipse $>,<$ rect $>,<$ polyline $>$, and $<$ polygon $>$. Each one of these shapes has its values and attributes that enable controlling them Dailey et al., 2012. All the shapes that stated in the examples below is shown in Figure 41. 


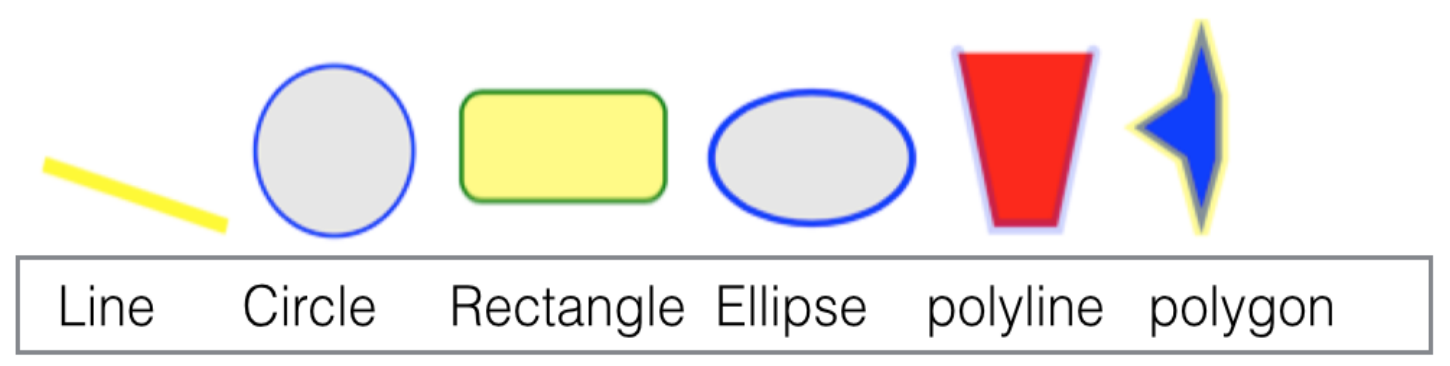

Figure 41: Basic vector shapes for SVG.

Line: has $(x 1, y 1)$ values as starting coordinate from the upper-left corner of the screen which is $(0,0)$, and $(x 2, y 2)$ values as ending coordinates. For instance:

$<$ line $x 1=" 10 " y 1=" 10 " x 2=" 150 "$ y2="50" stroke = "yellow" stroke-width $=" 10 ">$. This means the line starting coordinate from the upper-left corner of the screen will be at $(10,10)$ point while the ending coordinate point will be at $(150,50)$. The colour of the line is yellow with 10 pixels width.

Circle: has $(c x, c y)$ values for the centre and $r$ for the radius. For instance: $<$ circle $c x=" 150 " c y=" 150 " r=" 100 "$ stroke="blue" stroke-width=" 5" fill=" grey" fill-opacity= "0.2" $>$. This means the circle centre will be at $(150,150)$ point from the upper-left corner of the screen, with 100 pixels radius. The circle outside stroke colour is blue with 5 pixels width. The inside filling is grey with .2 filling opacity.

Rectangle: has $(x, y)$ values as top-left corner coordinate and width, height values for the rectangle width and height. In addition, there are rx and ry to declare the corners rounding degree. For instance:

$<$ rect $x=" 50 " y=" 100 "$ width $=" 100 "$ height $=" 50 "$ rx="10" ry="10" stroke="green" strokewidth="2" fill="yellow" fill-opacity="0.6" > . This means $(50,100)$ point is the top-left corner coordinate of the rectangle, and 100, 50 are width and height values for the rectangle width and height respectively. In addition, 10 pixels are the corners rounding degree. The rectangle outside stroke colour is green with 2 pixel width. The inside filling is yellow with .6 filling opacity.

Ellipse: has $(c x, c y)$ values for the centre and $(r x, r y)$ for $(\mathrm{x}, \mathrm{y})$ radius values. For instance: $<$ ellipse $c x=" 150 "$ cy="150" ry="50" rx="70" stroke="blue" stroke-width="5" fill="grey" fill-opacity="0.2" $>$. This means the ellipse centre will be at $(150,150)$ point with 50 and 70 as $\mathrm{x}, \mathrm{y}$ radius respectively. The ellipse outside stroke colour is blue with 5 pixels width. The inside filling is grey with .2 filling opacity.

Polyline: it is similar to the line shape but with chain of the $x, y$ coordinates which will be drawn with open end unless the user add the closing line. For instance:

$<$ polyline points="200,60 240,230 310,230 350,60" fill="red" stroke="blue" stroke-width="15" stroke-linecap="round" stroke-opacity= "0.2" >. This means the polyline starting coordinate 
from the upper-left corner of the screen will be at $(200,60)$ point. This line will be connected with other line with the following coordinates respectively: $(240,230),(310,230)$ and $(350,60)$ at the end as ending coordinate point. The colour of the polyline stroke is blue with 15 pixels width. The stroke has round linecap with .2 opacity. The filling color between the starting and ending points is red.

Polygon: has $(x 1, y 1)$ values as starting coordinate and $(x 2, y 2)$ values as ending coordinates which will be drawn with closed ends automatically. For instance:

$<$ polygon points="100,50 115,120 115,180 100,250 85,180 50,150 85,120" fill= "blue" stroke= "yellow" stroke-width="10" stroke-opacity="0.5" stroke-linejoin="miter" $>$. This means the polygon starting coordinate from the upper-left corner of the screen will be at $(100,50)$ point. This line will be connected with other line with the following coordinates respectively: $(115,120)$, $(115,180),(100,250),(85,180),(50,150),(85,120)$ and $(350,60)$ at the end as ending coordinate point. The colour of the polygon stroke is yellow with 10 pixels width. The stroke has a miter shape to joint between two line segments with .5 opacity. The filling color is blue.

\subsubsection{Why SVG?}

SVG over the other image formats has many advantages. One of them comes from being a vector based, which is having a smaller file size. Therefore, SVG can be loaded and transferred across the Internet more quickly than other formats which makes SVG suitable for the web application that has massive images representation such as complex biological networks or trees. In addition to speed of transfer, SVG text is searchable. While text inside the raster file is joined with the image and considered as apart of the image itself, the text information inside the SVG images is searchable. For this matter, the text in Google indexes is saved in SVG format in the web Pilgrim, 2010]. This feature is beneficial in a genome studies. For example, searching in the web about specific protein domain that drawn as a text inside SVG image can show the protein domain architecture images for this domain.

In web graphics design, the choice of the type of multimedia images is depending a lot on the application itself and the main purpose of this image. In the biological images, it is important to resize and zoom-in/out some figures to see how much details that have as seen in Figure 42 . When raster images such as canvas $2 \mathrm{D}$ is used, the image should has high resolution with a large file size to prevent the pixelated edges. As a result, page-loading time is affected negatively. For this matter, SVG images will fit perfectly on the applications that give the resize and re-colour features high priority.

To conclude, SVG is a better choice for the biological applications that required high resolution than any other raster images for many reasons. One of the most important reasons is the easiness of scaling the images in SVG format at any monitor, small as cell phones or big as TV monitors, without fixed pixel size limitation. In contrast, modifying raster images are problematic, and if it happened the images will loose a lot of details after scaling Peng and Zhang, 2004. The other reason is that SVG file is based on XML file, which supports portability. That's mean the SVG 
image can be displayed and edited at any platform and environment regardless of any web browser or operating system.

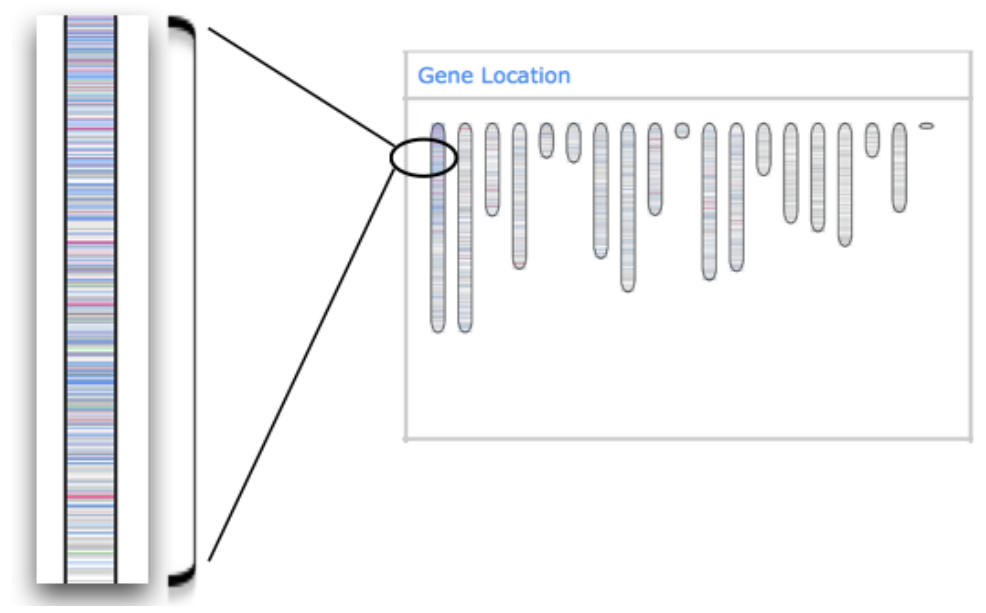

Figure 42: The image draws gene locations that locate in chromosome using scalable vector format as part of CGenome spreadsheet. It shows a small portion of gene location after scaling which is scaled in high quality without affecting the image clarity.

\subsubsection{JavaScript}

Unlike old-style static websites, modern websites should offer the user a full interaction features with the page contents. For that purpose, an interactive and strong programming language that has more flexible characteristics is becoming something desirable more than before.

Today, JavaScript (JS) is one of the most popular dynamic programming languages that mainly used with web browsers. Many computer-programming languages, JavaScript in our case, support the client-side scripts for directing the browser, enabling user interaction, displaying/altering dynamic document content. In addition to the client-side support, JavaScript supports the following: server-side programming using node.js application, mobile application creation, and game development Flanagan, 2011.

Back to the history, JavaScript was created by Netscape, which is now called Mozilla Foundation. It is one of the multi-paradigm languages that influenced by: C, Java, Python and scheme languages. It supports imperative, functional, scripting and object-oriented (OO) programming styles. JavaScript has the basic API for working with the following regular terminologies: text, arrays and dates. Since JavaScript does not include any input/output functionality, the I/O function is the responsibility of the host that embed the JavaScript codes, which particularly is a web browser such as HTML5. Using JavaScript makes the browser's responding to the user action much faster than other languages because the code runs in the client's browser not the server-side.

JavaScript is commonly used with HTML as embedded script with the element $<$ script $\rangle$ at the beginning of the script and ended by $</$ script $>$ element such as the below example. 


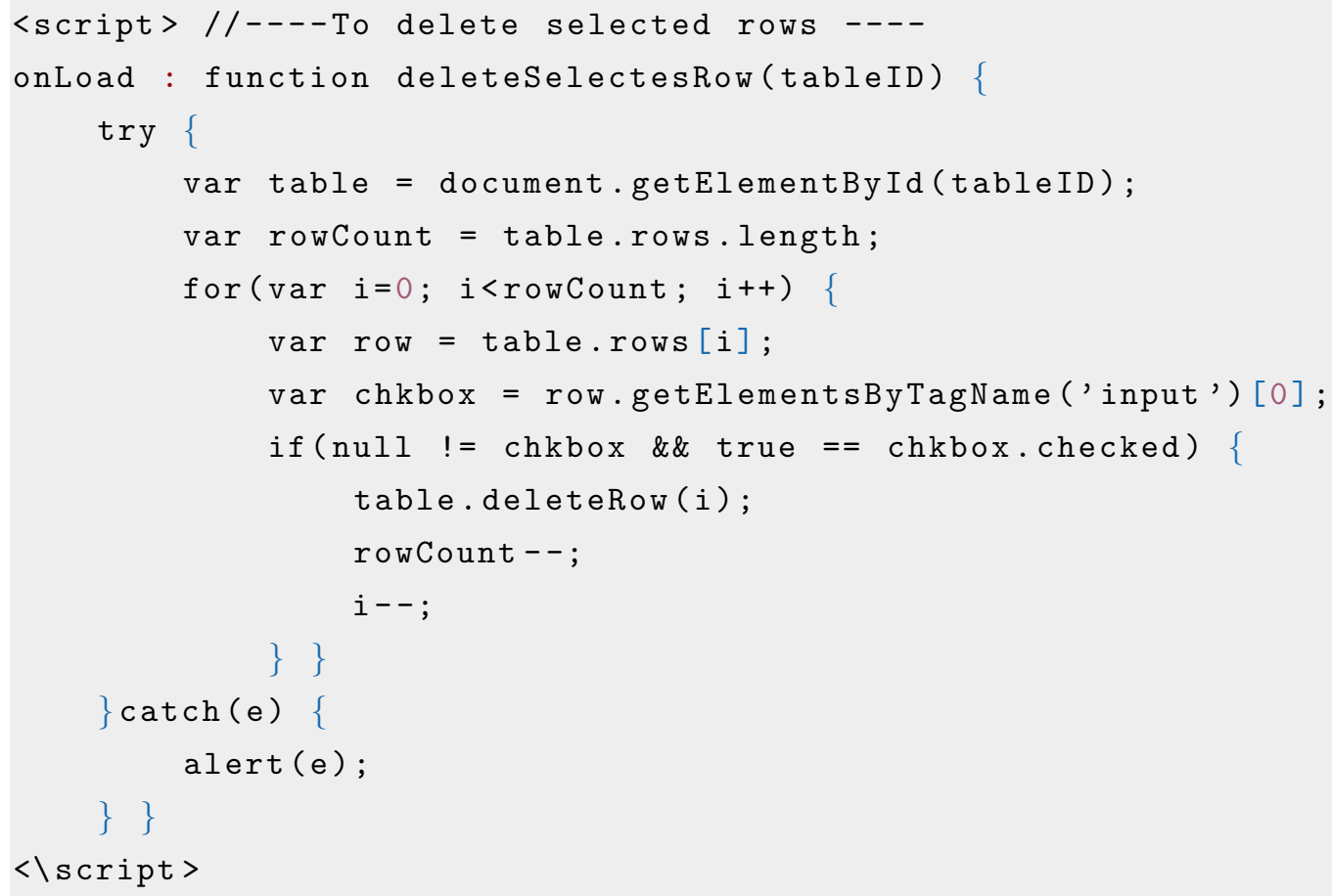

This embedded code used to supplement the client-side behaviour of HTML pages by interacting with the HTML Document Object Model (DOM).

Why JavaScript? There are many scripting languages that can be used but JavaScript is selected for having some important features. First feature, all modern browsers support and implement JavaScript natively such as embedding JS on HTML, described in the previous paragraph. Second, using JavaScript open the gates to take the advantages of using many popular and powerful JavaScript libraries that support visualization, such as jQuery and D3.js. Finally, JavaScript enhances the speed of the web browsers because its implementation does not require an internet connection since it uses its own Graphical User Interface (GUI). The others require plugins which needs online connectivity in order to generate the tree server-side.

\subsubsection{Why HTML 5, SVG and JavaScript?}

As known, SVG has many features, and the most important features in this research are having the ability to zoom-in/out without loosing the resolution, having small file size, having interactive feature, and having the support from almost all the web browsers except Internet Explorer v8 and older. Moreover, SVG elements can be controlled by CSS and JavaScript code in HTML5 environment Smits and Ouverney, 2010.

In biological web-application, the usage of JavaScript with some markup languages offers the webdesigners a complete and stable platform for building interactive webpages. For instance, HTML5 can be used as a template to identify the content of web pages, CSS to indicate the style that contents can be presented in the web pages, $\boldsymbol{S} \boldsymbol{V} \boldsymbol{G}$ to display and edit the genome resizable images, and JavaScript to control how the webpages contents will act Flanagan, 2011. 


\subsection{Graphical User Interface}

In our system, the master page has links for two kinds of spreadsheets. The links for CGene are divided into two kinds, which are genome spreadsheet with normalized images while the other is for the images that reflect the real coordinates of the exons, introns and protein domains. For both normalize and unnormalized links, there is a visual spreadsheet to present all genes in a specific genome. For each single gene, many properties are displayed such as gene ID, gene length, gene model, protein length and the protein domain architecture. For CGenome, there is only one link that directs to the comparative spreadsheet. In this spreadsheet, a comparative analysis for four different genomes is done by using three different visualizations, which shown in separate columns. The Gene location column is used to show the gene location on each chromosome. In the Go Slims bitmap column, the GO Slims occurrence in the specified genome is declared. Lastly, the GO Slims histogram column represents the number of occurrence of each GO Slim term.

The next Figures $43,44,45$ and 47 are illustration of the outcome of our visual spreadsheet system using HTML5 for the whole genome.

In Figure 43 is screenshot of the master page of the visual spreadsheet system using HTML5 for the whole genome. As we see there are multiple links for two different kind of spreadsheets: CGene and CGenome. In the upper box there is spreadsheet that has multiple links for CGene pages. In the column called "genome spreadsheet with normalized images" there is multiple links for different genomes. When these links are pressed, they will show spreadsheets that have normalized lengths images. On the other hand, in the column called "genome spreadsheet with real length images" there is multiple links for different genomes. When these links are pressed, they will show spreadsheets that have real lengths images. In the lower box there is a single link for CGenome page.

In Figures 44 and 45 are the two kind spreadsheets of CGene. In Figure 44 is spreadsheet with normalized images of the gene model and protein domains. On the other hand, in Figure 45 is the other kind of CGene spreadsheet, where all the images reflect the real coordinates of the exons, introns and protein domains. For both spreadsheets gene length and protein length are presented as number reflect the real length. The gene ID is represented textually. Any row can be selected using the check box in order to enable the user to have limited dataset. Figure 46 is a snapshot of $\boldsymbol{C G e n e}$ spreadsheet where it possible now to display more than $>10000$ genes in single spreadsheet.This

spreadsheet has normalized SVG images gene model and normalized protein domain architecture images.

Figure 47 shows a screenshot of single row of the comparative spreadsheet, which is for Aspergillus niger CBS 513.8 genome. In this spreadsheet, there is a comparative analysis for four different genomes which are Aspergillus fumigatus Af293, Aspergillus nidulans FGSC A4, Aspergillus niger CBS 513.8 and Aspergillus oryzae RIB40. This analysis is done using three different visualizations, which shown in separate columns. The Gene location column is used to show the gene location on each chromosome. In the Go Slims bitmap column, the GO Slims occurrence in the specified genome is declared. Lastly, the GO Slims histogram column represents the number of occurrence of each GO Slim term. 


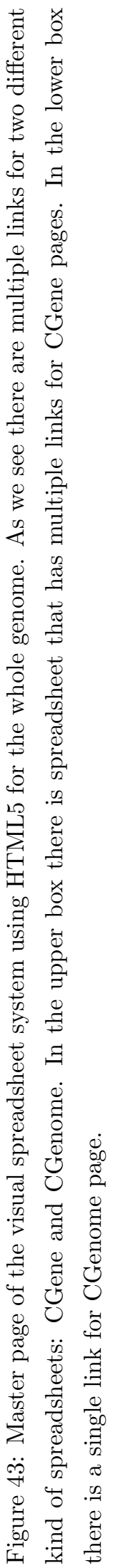




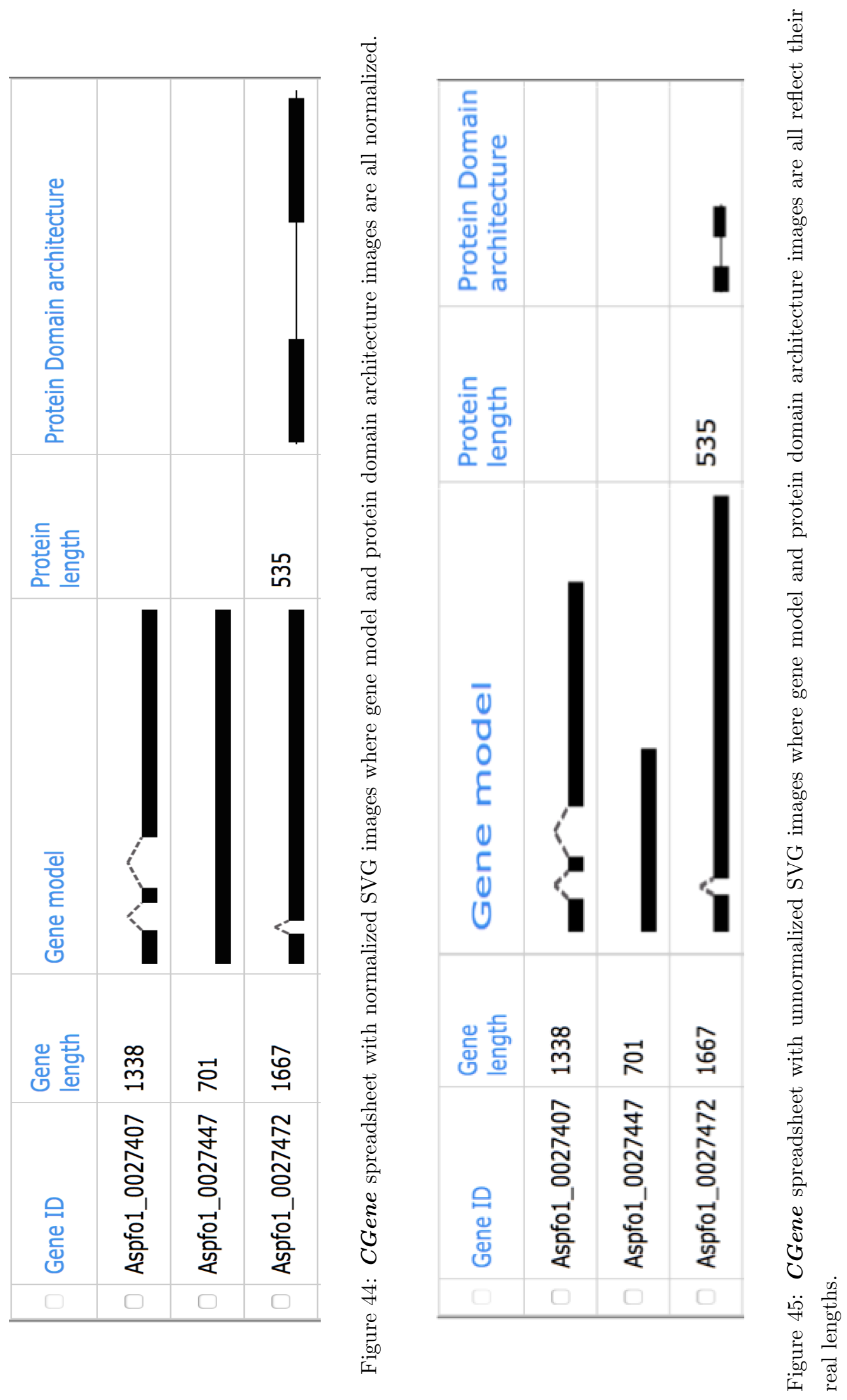



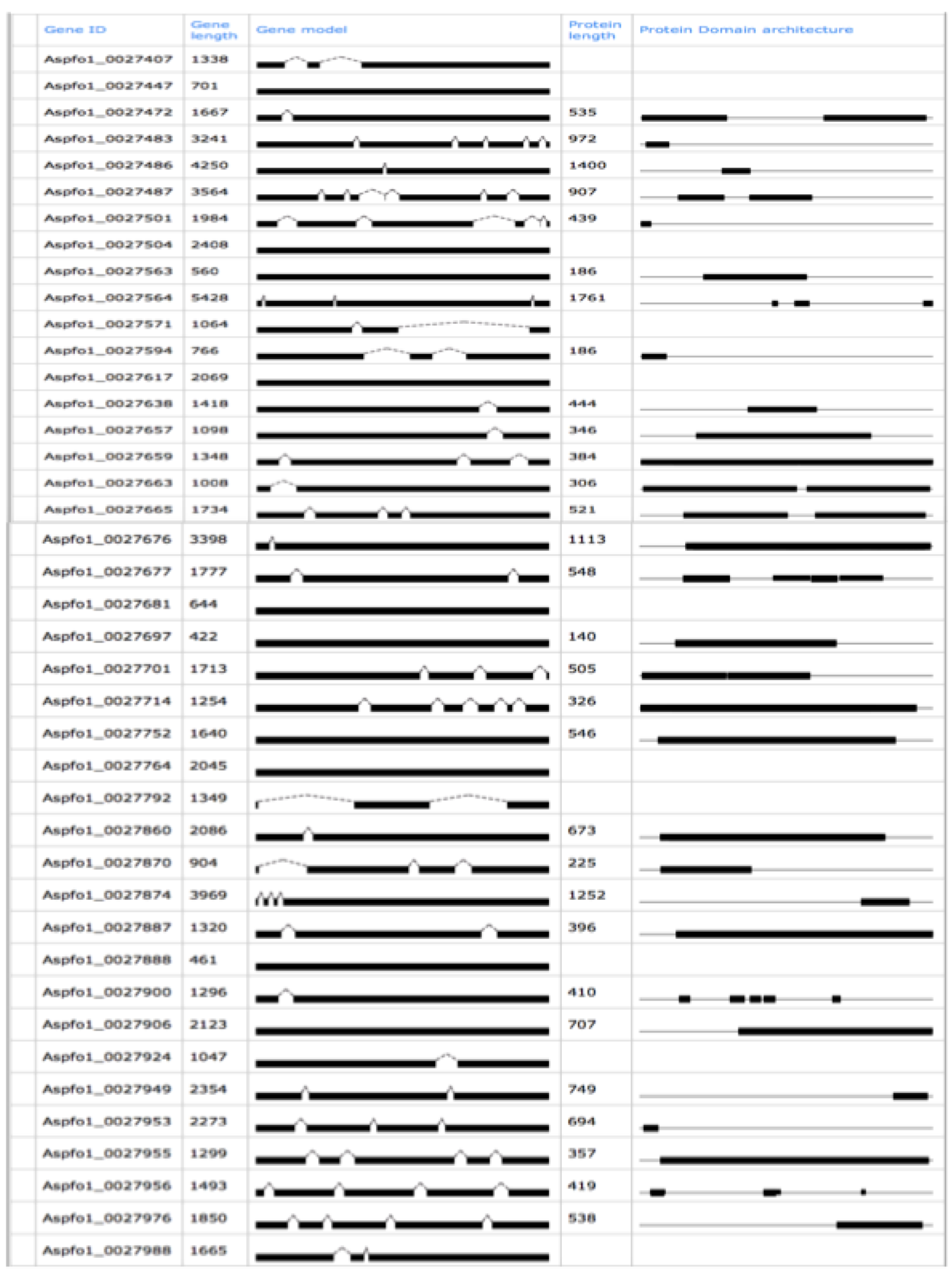

Figure 46: Snapshot of $\boldsymbol{C G e n e}$ spreadsheet where it possible now to display more than $>10000$ genes in single spreadsheet.This spreadsheet has normalized SVG images gene model and normalized protein domain architecture images. 


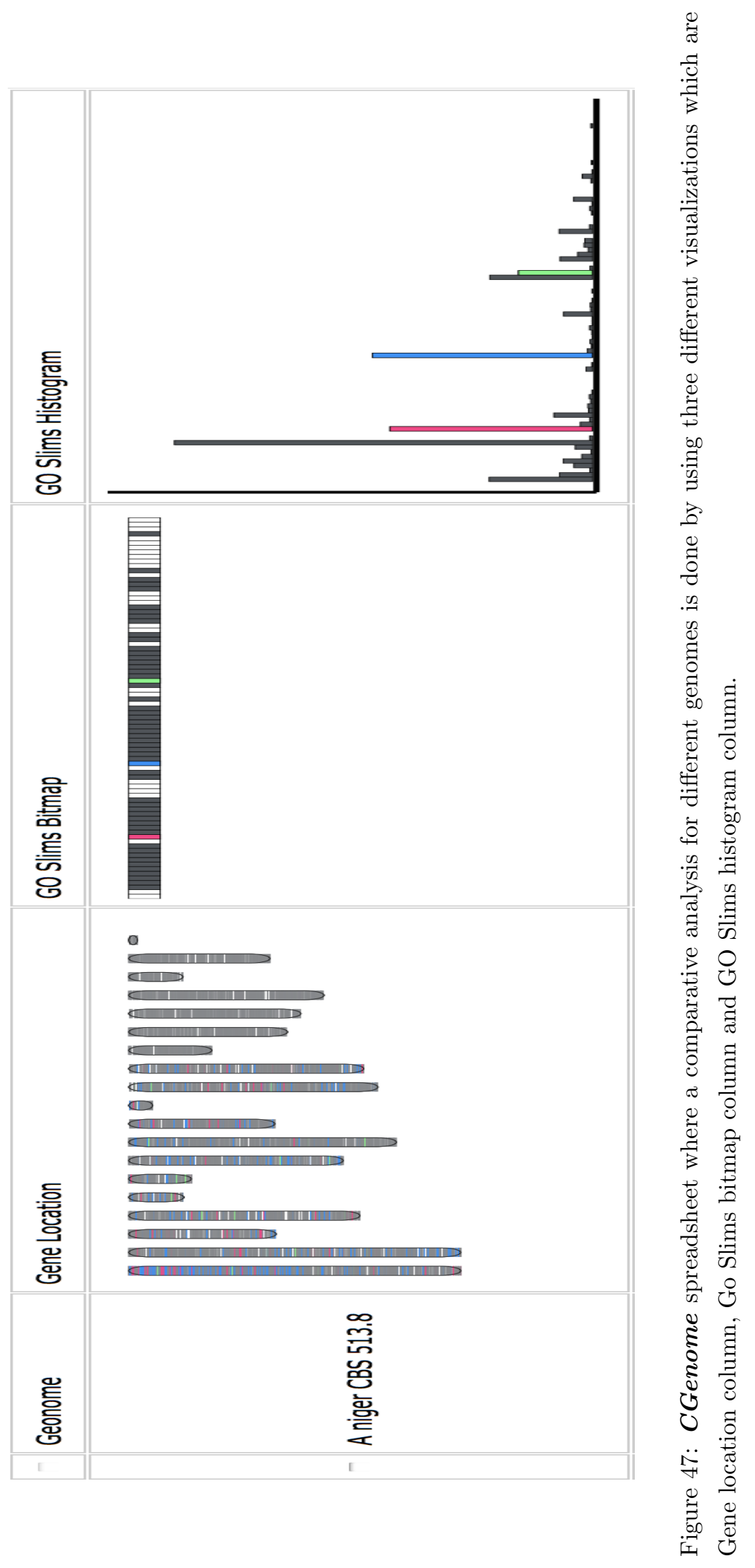




\section{Chapter 4}

\section{Conclusion and Future Work}

Visualization tool for presenting the whole genome annotation data is becoming more pressing, especially with the exponential and rapid development of genomic data. Analyzing the visual display of the gathered annotations enables better understanding for the genome and organism development. For the assessment, many scientists prefer observing the genome at high level, which is the level of an exon, a gene, and a biochemical pathway. Consequently, there are many special tools that created for visualizing the gene structure and its encoded regions, but many of them have limited visualizing options. For example, some of the tools are presenting single gene with its products in single view, while others are presenting limited number of genes in specific location on the genome such as genome browsers, which makes the whole genome assessment difficult. Consequently, there is a need to present all the genes in the genome associating with its encoded protein domains if found.

\subsection{Summary of Works}

In our work, we have implemented CGene and CGenome, pronounced See-Gene and See-Genome respectively, as a HTML5 web-based spreadsheets system. That makes the visualization of the whole genome annotation data in integrated visual displays, as well as text, within the spreadsheet cells possible.

We first analyzed different annotation files from the AspGD database ( www.aspgd.org ) and InterPro (http://www.ebi.ac.uk/interpro ) about Aspergillus fungal genomes with different file formats. These files include gene features, Protein Domain Predictions information, Gene Ontology (GO) annotations, AspGD chromosomal features and Go Slims description. Then, we extracted the key features that required for displaying the following: gene model, protein domains, genes location in the chromosome and GO Slims availability in the genome. We also did a comprehensive study about the protein domains on five of the common used Aspergillus fungal genome, which are A. nidulans, A. fumigatus, A. flavus, A. niger and A. oryzae. This study was to observe which are the top twenty-five repeated domains.

After that, we implemented two types of HTML5 spreadsheets using Java and JavaScript languages. The first spreadsheet, called CGene, is including the visual display for all the genes in single 
genome lined up with its related protein domain. This spreadsheet has some textual and visual SVG images about the genomes' genes and proteins in each row. The second spreadsheet, called CGenome, has different genomes lined up, in which each row in the spreadsheet represent single genome. In each row of the spreadsheet we display the following in SVG format: Gene location, Go Slims bitmap and Go Slims histogram. In both spreadsheets, some rows can be selected to be the only viewed dataset, and all the SVG images can be downloaded as SVG format for user personal use.

\subsection{Work Contributions}

As mentioned previously, there is a high demand for visual representation for all the genes in the genome to be in a single place. In our research, we put the annotated information about the gene and its encoded protein in a single place, and gather them in tabulate view to allow users to explore patterns much easier. Most of the efforts that are done previously in the tabular/spreadsheet layout were to find a universal format to exchange "omics" data/metadata and experiment results. In our system we implemented two visual spreadsheets. One called CGene that used to represent single organism while the other, which called CGenome, used to represent multiple genomes.

In CGene, we created a spreadsheet in which users are able to visualize more than 10000 genes of single organism in single spreadsheet. In addition of summarizing all these genes in a one place, they are presented visually in SVG format. Some of the genes can be selected as dataset or/and the images can be downloaded for users further study. There is no such bioinformatics system that organizes data of thousands of genes in a single spreadsheet visually.

In CGenome, we created a spreadsheet in which users are able to compare different organisms by using three different visualizations. These visualizations are Gene location, Go Slims bitmap and GO Slims histogram. Gene location column is used to show the gene location on each chromosome where the genes are colored in pink, green and blue based on the three gene ontology aspects; One

color for each aspect. This will allow the user to see the distribution of the GO Slims over the chromosomes. In Go Slims bitmap column, GO slims availability in the specified genome is shown. GO Slims histogram column that used to illustrate the distribution of the dataset by representing the number of occurrence of each GO Slim term. Any of those images can be selected and downloaded for users further study.

By presenting that information visually the scientists can use this spreadsheet to gain some understanding about the data collected about a whole genome such as genes, proteins, annotations, and expression data.

\subsection{Research Limitations and Future Work}

Although we implemented an HTML5 spreadsheet for the whole genome, the created spreadsheet is fixed, in which the user can browse, select dataset, and download specific SVG images. There is no flexible customization while creating the spreadsheet. The other limitation is about protein domain 
architecture, since there is no clear standard about how to visualize protein domains. Different systems are using different colours and shapes to represent protein domains architecture. As a result of having hundreds of domains without having a standard about visualizing them, we decided to display them in rectangle shape with black colour and the top 25 repeated domains could be displayed in different colours.

One of the limitations that need to be stated is the lack of system evaluation. We did not evaluate the system since our goal at the beginning was to create a stable visualization system as a starting point.

Despite the fact that many information visualization evaluation techniques became widespread, the process of evaluating information visualization interfaces still complicated process Lam et al., 2011]. When a new technology is presented, some early adaptors, who like to try any new features, will use this technology straightway. The challenge is how this technology reaches the early majority users who care about systems that are reliable, useful and solve problems Plaisant, 2004. Information visualization is one of the technologies having utility evaluation as major challenge Plaisant, 2004. Moreover, the current information visualization evaluation techniques have many weaknesses such as buggy implementation of new software, testing the wrong users and users familiarity with the traditional interfaces Andrews, 2006. As a result, it is unfair to compare new-implemented interfaces with tested and familiar interfaces since the users will prefer the familiar one. In addition, the wrong users such as computer science users test many visualization systems, which leads to inaccurate results. The users are better to be real users such as intelligence analysts or pharmaceutical.

According to Andrews, 2006, to evaluate the information visualization systems, there are three common testing methods which are summative test, formative tests, and usage tests. Formative testing requires generally observing a small group of test users such as three to five. After the observation, there will be an idea about what and why problems happen. One of the formative testing techniques is thinking aloud where the users are asked to give their opinions while they are using the system. This kind of test is useful during visualization system development in order to fix the design and implementation bugs. The result of this test cannot be generalized to other visualization systems because the occurred problems are related to specific tasks and the number of the test group is small. In thinking aloud test, some usability attributes are not measured such as task completion time and effectiveness. Both of them require formal experiment to be measured. In contrast, Summative testing requires data measurements such as ask completion time, number of clicks and many statistical analysis. One of summative testing techniques is formal experiment. To compare between multiple interfaces, these experiments can be designed into two ways, which are between-groups and within-groups experiment. In between-groups experiment, each group is responsible on testing one interface. In contrast, one group tests multiple interfaces in withingroups experiment. Within-groups are commonly used because it requires less test users and reduces the individual differences while testing different interfaces. The number of the test users is usually between 50-100. The third method that used for evaluating the visualization system is usage studies. This method requires observing a group of test users while working on the interface for long time. Most usage studies depend on self-reporting where users creating a log about what they did, what 
is happened, and how long time it takes. This way is time consuming but it is useful for studying part of the software or for comparing between two or more interfaces.

In addition to the previous methods, one of the ways that can determine the success/failure of a website is using user-centric method while designing the software, which can be done by using personas. Persona as Cooper defined is "a fictitious, specific and concrete representation of target users" Cooper, Alan and others, 1999. Personas will act as stand-ins for real users, which can help to guide decisions about software functionality and design. By defining system personas, developer can reference the users' needs and ensure a more useful and successful software. The designer also can evaluate the new site feature ideas based on users' goals and needs. We have created three personas to represent the users that may use our system, which are: Carolyn Smith, Salem Hadi and Kumar Amit. We can determine the usability of the system by knowing the users motivation to use our system and implementing their needs.

Carolyn Smith is a Biologist in BioVis Lab. She is 30 years old and single. She has a master's degree in Bioinformatics Engineering. Carolyn is goal-oriented and focused person with good team management skills. One of her concerns is data analysis and visualization. She spent her work time on collecting data, visualizing them and performing experimental tests for data visualization especially on eukaryote organisms. She focused in genes functionality and GO Slims information. Her workplace is connected with wifi. She works in the laboratory and performs various experiments in Aspergillus genome research. She works 9 hours a day and keeps log for every activity and task that is done. She has project about Aspergillosis, which is the group of diseases caused by Aspergillus organisms. In this project, she has to identify some of unknown genes functions. She focused on an experiment that states the gene function can be identified by knowing the gene location and the Gene Ontology Slim information from neighbouring genes Amthauer and Tsatsoulis, 2010. Since getting information from images is less time-consuming than looking through numbers and texts, she needs software that helps her to visualize the genes location in the chromosome and shows the GO Slims distribution in the chromosome. This software can help her to discover the interested gene and to identify its function by knowing the gene location and GO Slim information of a gene's two-nearest neighbouring genes. She needs to download this image with high resolution on her own device in case she wants to send the result to her lab mates by email. The high-resolution scalable image for the chromosome will help her to see the gene location among hundred of genes in the chromosome .

Salem Hadi is a bioinformatics researcher at King Khaled University. He is 25 years old and single. He has a bachelor's degree in Computer Science. Hamad is focused person with good communication skills. One of his concerns is genomic data analysis and visualization. He spent his work time on collecting data, analyzing them and finding software to visualize them. His group research focused on studying fungal genomes especially the Aspergilli genomes. He focused in genes annotation. His workplace is connected with wifi. His work is mainly on visualizing the annotated data. He works 9 hours a day and keeps log for every activity and task that is done. He needs a tool to visualize the extracted gene features alongside with its coding protein. He wants to study specific genes on selected Aspergillus genome. He needs a tool help him to compare between different genes. When 
he finishes comparing between genes, he wants to download the gene model and the protein domain architecture images in high resolution so he can post it on his paper research.

Kumar Amit is bioinformatics $\mathrm{PhD}$ full time student at Concordia University. He is 30 years old and married. He has a master's degree in Bioinformatics Engineering. He has strong communication skills and had an experience on teaching. One of his concerns is genomic comparative analysis. His research requires performing a comparative analysis between different genomes and one of them is Aspergillus genomes. He works on a poster about comparative analysis between genomes in order to present it on a conference. He needs an example to represent on his poster with scalable images that does not loos its resolution when scaling. This example should have multiple genomes compared in different aspects and one of them is showing gene location on the chromosomes.

Future Work As the system has some limitations as noted previously, we consider some suggestions for a further improvement to the system.

First, apply some evaluation techniques on the system to fix the bugs and to improve the system utility. To evaluate CGene and CGenome, we need to include a biologists on the testing group since they are one of the main targeted users. As a result, we can have an idea about the system usage and easiness. We will be able to measure how much biologist familiar with our system comparing to the available system. This can be done by applying some of evaluation methods on information visualization stated previously. Since CGene and CGenome are both HTML5 webpages, the website usage can be studied where a users usage data are recorded for period of time such as 30 days. After recording this log, we can have an idea about what are users looking for and how much time they spend on each interface. We will count how many users used the website and for how long. We can have an idea about their satisfaction by offering online survey or by observing if they return back or not. We also can apply within-groups experiment to test every interface. From this experiment we can have an idea about how many clicks the user needs to browse the spreadsheet, select dataset and to download SVG images. We will also have an idea about how much time is required for browsing and downloading dataset. The testing group should log the bugs that may happen during browsing, selecting some rows and downloading images.

Second, support customization by engaging the user during the creation of the spreadsheet. By applying this way the settings done by specific user will not affect another user's setting since the changes will be applied to the user outcome on its local device. Consequently, more than one user can use the same system but the outcome may differ based on individual settings.

Third, support configuration files since it will offer more power and flexibility to the user. By using configuration files during the creation of the spreadsheet, the user can set its own preference such as the colours or the shape of the protein domains, which makes noticing patterns in the spreadsheet easer. There are some suggested user-configuration options which can be applied in the future. For example, making SVG images for both gene model and protein domain architecture configurable to be normalized images or to be images that reflect the real length. The SVG images also can be coloured based on user preferences. The configuration could include putting specific colour or shape for specific protein domains, and configuring the spreadsheet schema for the requested organism such as setting the width/height of the table and setting spreadsheet columns' names. 
In addition to customization and configuration file support, there are some of spreadsheet operations that can be added. These operations include keyword search, pattern search, filtering, sorting, and clustering of columns containing visualizations. By adding those operations the user can search for specific keyword or pattern where the pattern could be a regular expression or a simple string. For example users can search for a gene whose name includes a particular string. On filtering the user can select certain rows based on certain criteria such as selecting all genes whose length value smaller or greater than a specified value. By using sorting operation the user can sort the spreadsheet rows based on the selected columns such sorting the rows based on gene length from low to high. On clustering, the rows can be grouped based on columns containing visualizations such as grouping the genes based on having specific domains.

\subsection{Resource of the system}

The codes that used to create CGene and CGenome have uploaded on Google subversion server that powered by Google Project Hosting. The code can be accessed on the address: https://a-visual -spreadsheet-using-html5-for-whole-genome-display.googlecode.com/svn/trunk/. 


\section{Bibliography}

[Albà et al., 2007] Albà, M., Tompa, P., and Veitia, R. (2007). Amino acid repeats and the structure and evolution of proteins.

[Amthauer and Tsatsoulis, 2010] Amthauer, H. A. and Tsatsoulis, C. (2010). Classifying genes to the correct Gene Ontology Slim term in Saccharomyces cerevisiae using neighbouring genes with classification learning. BMC Genomics, 11(1):340.

[Andrews, 2006] Andrews, K. (2006). Evaluating Information Visualizations. In Proceedings of the 2006 AVI workshop on Beyond time and errors: novel evaluation methods for information visualization, pages $1-5$. ACM.

[AspGD, 2014] AspGD (2014). Available at: www.aspgd.org.

[Biologyreference., 2014] Biologyreference. (2014). Available at: http://www.biologyreference.c om/Po-Re/Protein-Structure.html.

[Brehmer and Munzner, 2013] Brehmer, M. and Munzner, T. (2013). A multi-level typology of abstract visualization tasks. IEEE Trans. Visualization and Computer Graphics (TVCG) (Proc. InfoVis), 19(12):2376-2385.

[Card et al., 1999] Card, S. K., Mackinlay, J. D., and Shneiderman, B. (1999). Readings in information visualization: using vision to think. Morgan Kaufmann.

[Cooper, Alan and others, 1999] Cooper, Alan and others (1999). Inmates Are Running the Asylum, The: Why High-Tech Products Drive Us Crazy and How to Restore the Sanity, volume 261. Sams Indianapolis.

[Crick et al., 1970] Crick, F. et al. (1970). Central dogma of molecular biology. Nature, 227(5258):561-563.

[Dailey et al., 2012] Dailey, D., Frost, J., and Strazzullo, D. (2012). Building web applications with $S V G$. O’Reilly Media, Inc.

[Deacon, 2009] Deacon, J. (2009). Model-View-Controller (MVC) architecture. Online: http://www.jdl.co.uk/briefings/MVC.pdf. 
[Dudley and Karczewski, 2013] Dudley, J. T. and Karczewski, K. J. (2013). Exploring personal genomics. Oxford University Press.

[EMBL-EBI, 2014] EMBL-EBI (2014). Available at: http://www.ebi.ac.uk/training/online/ course/introduction-protein-classification-ebi/what-are-protein-signatures/signa ture-types/what-ar-2

[Fawal et al., 2012] Fawal, N., Savelli, B., Dunand, C., and Mathé, C. (2012). GECA: a fast tool for gene evolution and conservation analysis in eukaryotic protein families. Bioinformatics, 28(10):1398-1399.

[Flanagan, 2011] Flanagan, D. (2011). JavaScript: The definitive guide: Activate your web pages. O'Reilly Media, Inc.

[Frech et al., 2012] Frech, C., Choo, C., and Chen, N. (2012). Featurestack: Perl module for comparative visualization of gene features. Bioinformatics, 28(23):3137-3138.

[Gehlenborg et al., 2010] Gehlenborg, N., O’Donoghue, S. I., Baliga, N. S., Goesmann, A., Hibbs, M. A., Kitano, H., Kohlbacher, O., Neuweger, H., Schneider, R., Tenenbaum, D., et al. (2010). Visualization of omics data for systems biology. Nature Methods, 7:S56-S68.

[Gene Ontology Consortium and others, 2008] Gene Ontology Consortium and others (2008). The Gene Ontology project in 2008. Nucleic Acids Research, 36(suppl 1):D440-D444.

[Gene Ontology Consortium and others, 2010] Gene Ontology Consortium and others (2010). The Gene Ontology in 2010: extensions and refinements. Nucleic Acids Research, 38(suppl 1):D331D335.

[GeneOntology, 2014] GeneOntology (2014). Gene Ontology Consortium. Available at: http://ge neontology.org/page/download-ontology.

[Hertz and Stormo, 1999] Hertz, G. Z. and Stormo, G. D. (1999). Identifying DNA and protein patterns with statistically significant alignments of multiple sequences. Bioinformatics, 15(7):563577 .

[Hrmova and Fincher, 2009] Hrmova, M. and Fincher, G. B. (2009). Functional genomics and structural biology in the definition of gene function. In Plant Genomics, pages 199-227. Springer.

[HTML5, 2014] HTML5 (2014). Available at: http://www.w3schools.com/html/htm15_intro.a $\mathrm{sp}$.

[InterPro, 2014] InterPro (2014). InterPro DB. Available at: http://www.ebi.ac.uk/interpro

[Jameson et al., 2011] Jameson, D., Westerhoff, H. V., and Verma, M. (2011). Methods in systems biology, volume 500. Academic Press. 
[Kent et al., 2002] Kent, W. J., Sugnet, C. W., Furey, T. S., Roskin, K. M., Pringle, T. H., Zahler, A. M., and Haussler, D. (2002). The human genome browser at UCSC. Genome Research, 12(6):996-1006.

[Koch, 2011] Koch, R. (2011). The 80/20 principle: the secret to achieving more with less. Random House LLC.

[Koonin EV, 2003] Koonin EV, G. M. (2003). Sequence-Evolution-Function: Computational Approaches in Comparative Genomics. Kluwer Academic, Boston.

[Krzywinski, 2013] Krzywinski, M. (2013). Points of view: Elements of visual style. Nature Methods, 10(5):371-371.

[Lam et al., 2011] Lam, H., Bertini, E., Isenberg, P., Plaisant, C., Carpendale, S., et al. (2011). Seven Guiding Scenarios for Information Visualization Evaluation. Technical Report 2011-992-04, University of Calgary.

[Larman, 2012] Larman, C. (2012). Applying UML and Patterns: An Introduction to ObjectOriented Analysis and Design and Iterative Development, 3/e. Pearson Education India.

[Latham and Latham, 1995] Latham, R. and Latham, R. (1995). The dictionary of computer graphics and virtual reality, volume 2 . Springer.

[Letunic and Bork, 2007] Letunic, I. and Bork, P. (2007). Interactive tree of life (iTOL): an online tool for phylogenetic tree display and annotation. Bioinformatics, 23(1):127-128.

[Letunic and Bork, 2011] Letunic, I. and Bork, P. (2011). Interactive tree of life v2: online annotation and display of phylogenetic trees made easy. Nucleic Acids Research, 39:W475-W478.

[Luo and Nijveen, 2013] Luo, H. and Nijveen, H. (2013). Understanding and identifying amino acid repeats. Briefings in Bioinformatics, page bbt003.

[Majoros, 2007] Majoros, W. H. (2007). Methods for computational gene prediction, volume 1. Cambridge University Press Cambridge.

[Meyer et al., 2010] Meyer, M., Munzner, T., DePace, A., and Pfister, H. (2010). MulteeSum: A tool for comparative spatial and temporal gene expression data. IEEE Transactions on Visualization and Computer Graphics, 16(6):908-917.

[Morrison, 2013] Morrison, D. A. (2013). Evolutionary Genomics: Statistical and Computational Methods. Volumes 1 and 2. Systematic Biology, 62(2):348-350.

[Munzner, 2014] Munzner, T. (November 17, 2014). Visualization Analysis and Design. AK Peters Visualization Series. A K Peters/CRC Press, 1 edition.

[Nielsen et al., 2010] Nielsen, C. B., Cantor, M., Dubchak, I., Gordon, D., and Wang, T. (2010). Visualizing genomes: techniques and challenges. Nature Methods, 7:S5-S15. 
[O'Donoghue et al., 2010] O'Donoghue, S. I., Gavin, A.-C., Gehlenborg, N., Goodsell, D. S., Hériché, J.-K., Nielsen, C. B., North, C., Olson, A. J., Procter, J. B., Shattuck, D. W., et al. (2010). Visualizing biological data — now and in the future. Nature Methods, 7:S2-S4.

[Pavlopoulos et al., 2008] Pavlopoulos, G. A., Wegener, A.-L., and Schneider, R. (2008). A survey of visualization tools for biological network analysis. Biodata Mining, 1(1):1-11.

[Peng and Zhang, 2004] Peng, Z.-R. and Zhang, C. (2004). The roles of geography markup language (GML), scalable vector graphics (SVG), and web feature service (WFS) specifications in the development of internet geographic information systems (GIS). Journal of Geographical Systems, $6(2): 95-116$.

[Peter et al., 2004] Peter, B. J., Kent, H. M., Mills, I. G., Vallis, Y., Butler, P. J. G., Evans, P. R., and McMahon, H. T. (2004). Bar domains as sensors of membrane curvature: the amphiphysin bar structure. Science, 303(5657):495-499.

[Pilgrim, 2010] Pilgrim, M. (2010). HTML5: Up and Running. O'Reilly Media, Inc.

[Plaisant, 2004] Plaisant, C. (2004). The Challenge of Information Visualization Evaluation. In Proceedings of the Working conference on Advanced Visual Interfaces, pages 109-116. ACM.

[Rambaldi and Ciccarelli, 2009] Rambaldi, D. and Ciccarelli, F. D. (2009). FancyGene: dynamic visualization of gene structures and protein domain architectures on genomic loci. Bioinformatics, 25(17):2281-2282.

[Rangwala and Karypis, 2010] Rangwala, H. and Karypis, G. (2010). Introduction to Protein Structure Prediction: Methods and Algorithms, volume 14. John Wiley \& Sons.

[Ren et al., 2009] Ren, J., Wen, L., Gao, X., Jin, C., Xue, Y., and Yao, X. (2009). DOG 1.0: illustrator of protein domain structures. Cell Research, 19(2):271-273.

[Roberts, 2007] Roberts, J. C. (2007). State of the art: Coordinated \& multiple views in exploratory visualization. In fifth International Conference on Coordinated and Multiple Views in Exploratory Visualization, 200\%, pages 61-71. IEEE.

[Rouzé et al., 1999] Rouzé, P., Pavy, N., and Rombauts, S. (1999). Genome annotation: which tools do we have for it? Current Opinion in Plant Biology, 2(2):90-95.

[Saben et al., 2014] Saben, J., Zhong, Y., McKelvey, S., Dajani, N., Andres, A., Badger, T., GomezAcevedo, H., and Shankar, K. (2014). A comprehensive analysis of the human placenta transcriptome. Placenta, 35(2):125-131.

[Sanger-Institute, 2014] Sanger-Institute (2014). Gff: an exchange format for feature description gff: an exchange format for feature description. Available at: http://www.sanger.ac.uk/resources /software/gff/ 
[Sansone et al., 2008] Sansone, S.-A., Rocca-Serra, P., Brandizi, M., Brazma, A., Field, D., Fostel, J., Garrow, A. G., Gilbert, J., Goodsaid, F., Hardy, N., et al. (2008). The first RSBI (ISA-TAB) workshop: "can a simple format work for complex studies?". OMICS A Journal of Integrative Biology, 12(2):143-149.

[Schultz et al., 2000] Schultz, J., Copley, R. R., Doerks, T., Ponting, C. P., and Bork, P. (2000). SMART: a web-based tool for the study of genetically mobile domains. Nucleic Acids Research, 28(1):231-234.

[Shannon et al., 2003] Shannon, P., Markiel, A., Ozier, O., Baliga, N. S., Wang, J. T., Ramage, D., Amin, N., Schwikowski, B., and Ideker, T. (2003). Cytoscape: a software environment for integrated models of biomolecular interaction networks. Genome Research, 13(11):2498-2504.

[Smits and Ouverney, 2010] Smits, S. A. and Ouverney, C. C. (2010). jsPhyloSVG: a javascript library for visualizing interactive and vector-based phylogenetic trees on the web. PloS One, $5(8): \mathrm{e} 12267$.

[Soltis and Soltis, 2003] Soltis, D. E. and Soltis, P. S. (2003). The role of phylogenetics in comparative genetics. Plant Physiology, 132(4):1790-1800.

[Stein, 2001] Stein, L. (2001). Genome annotation: from sequence to biology. Nature Reviews Genetics, 2(7):493-503.

[Tufte and Graves-Morris, 1983] Tufte, E. R. and Graves-Morris, P. (1983). The visual display of quantitative information, volume 2. Graphics Press, Cheshire, CT.

[Wang Baldonado et al., 2000] Wang Baldonado, M. Q., Woodruff, A., and Kuchinsky, A. (2000). Guidelines for using multiple views in information visualization. In Proceedings of the Working Conference on Advanced Visual Interfaces, pages 110-119. ACM.

[Watson et al., 1953] Watson, J. D., Crick, F. H., et al. (1953). Molecular structure of nucleic acids. Nature, 171(4356):737-738.

[Wikipedia, 2014] Wikipedia (2014). Available at: http://en.wikipedia.org/wiki/Protein_fo lding

[Wong, 2012] Wong, B. (2012). Points of view: Visualizing biological data. Nature Methods, $9(12): 1131-1131$. 
Appendix A

Gene Model Catalog 


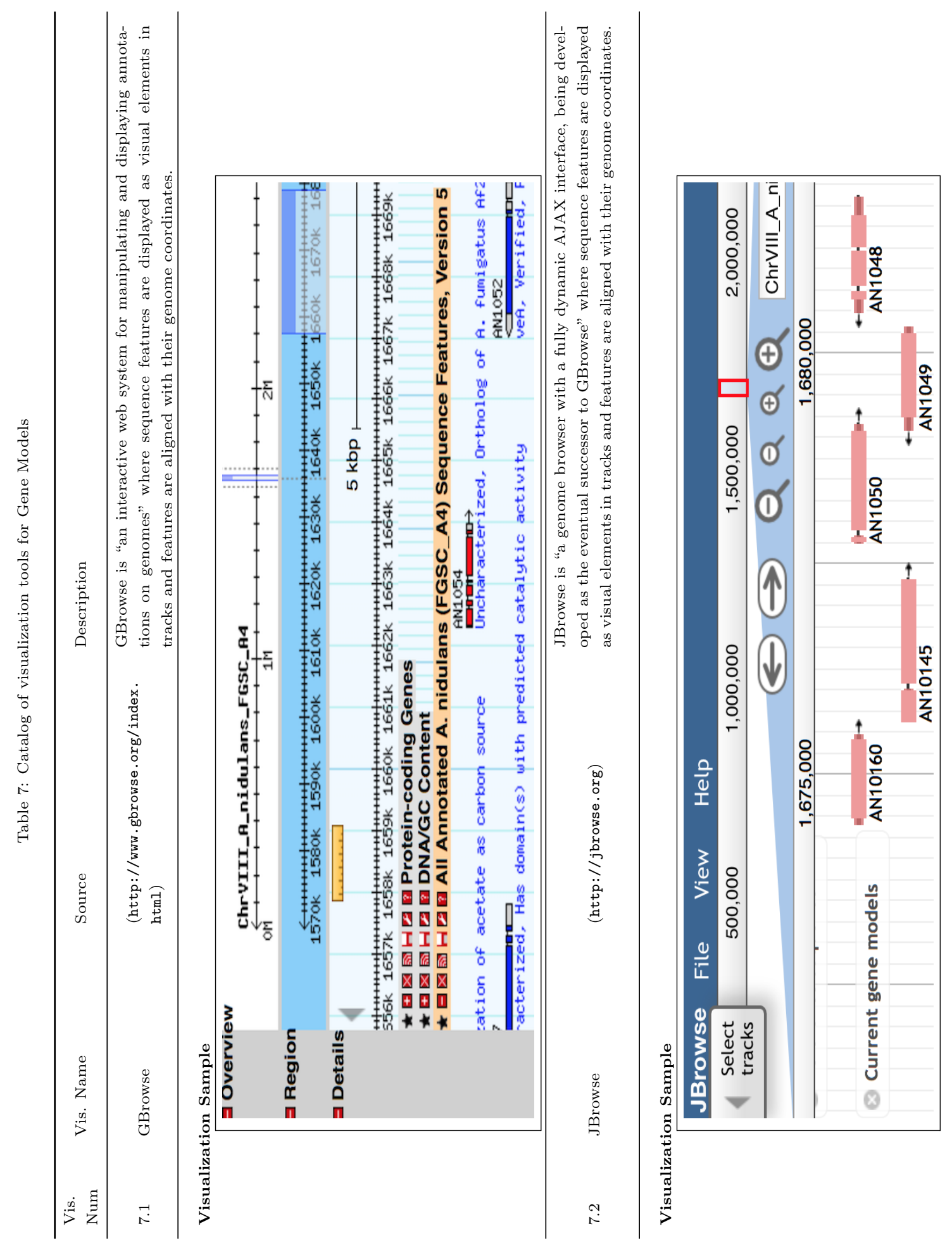




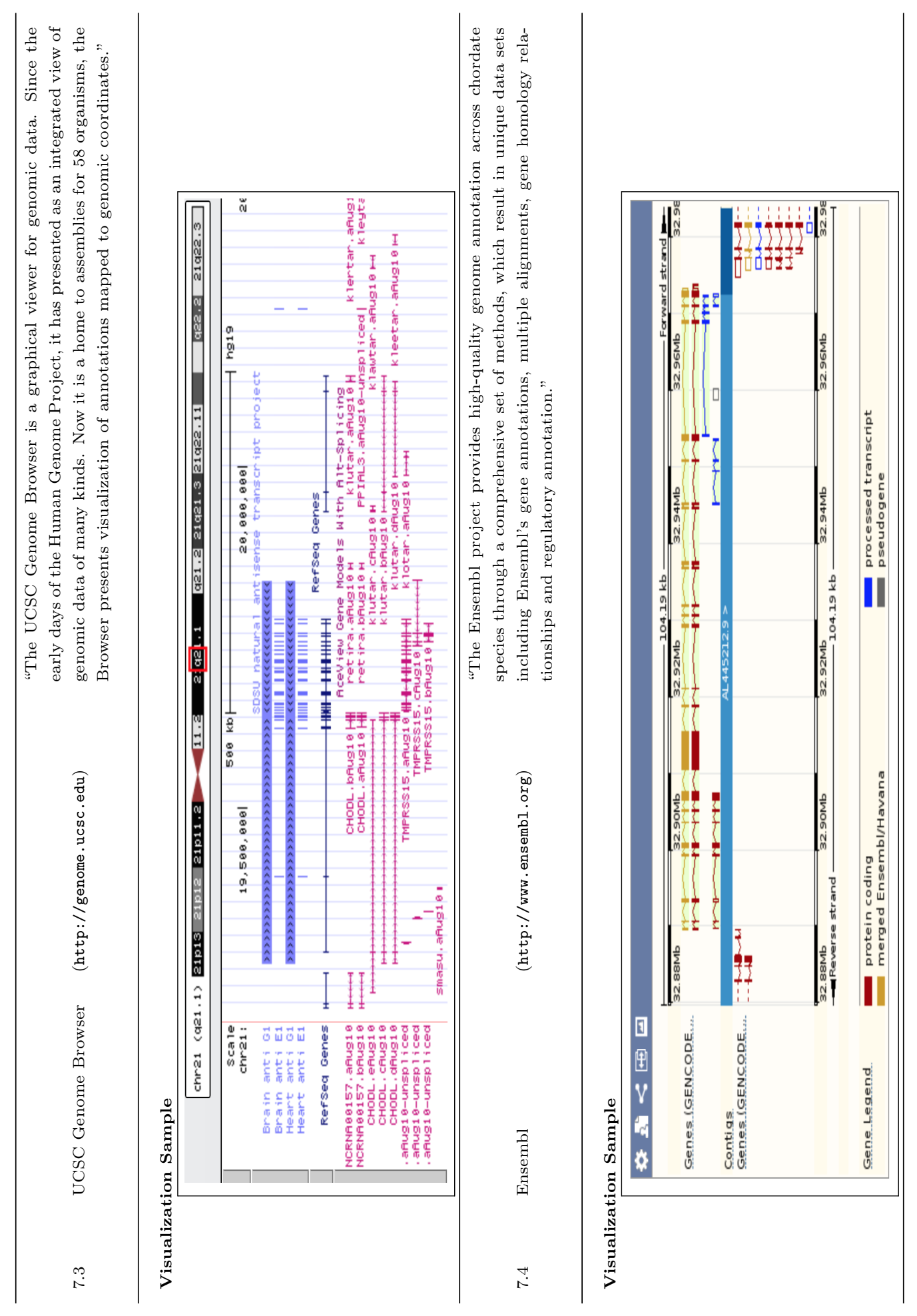




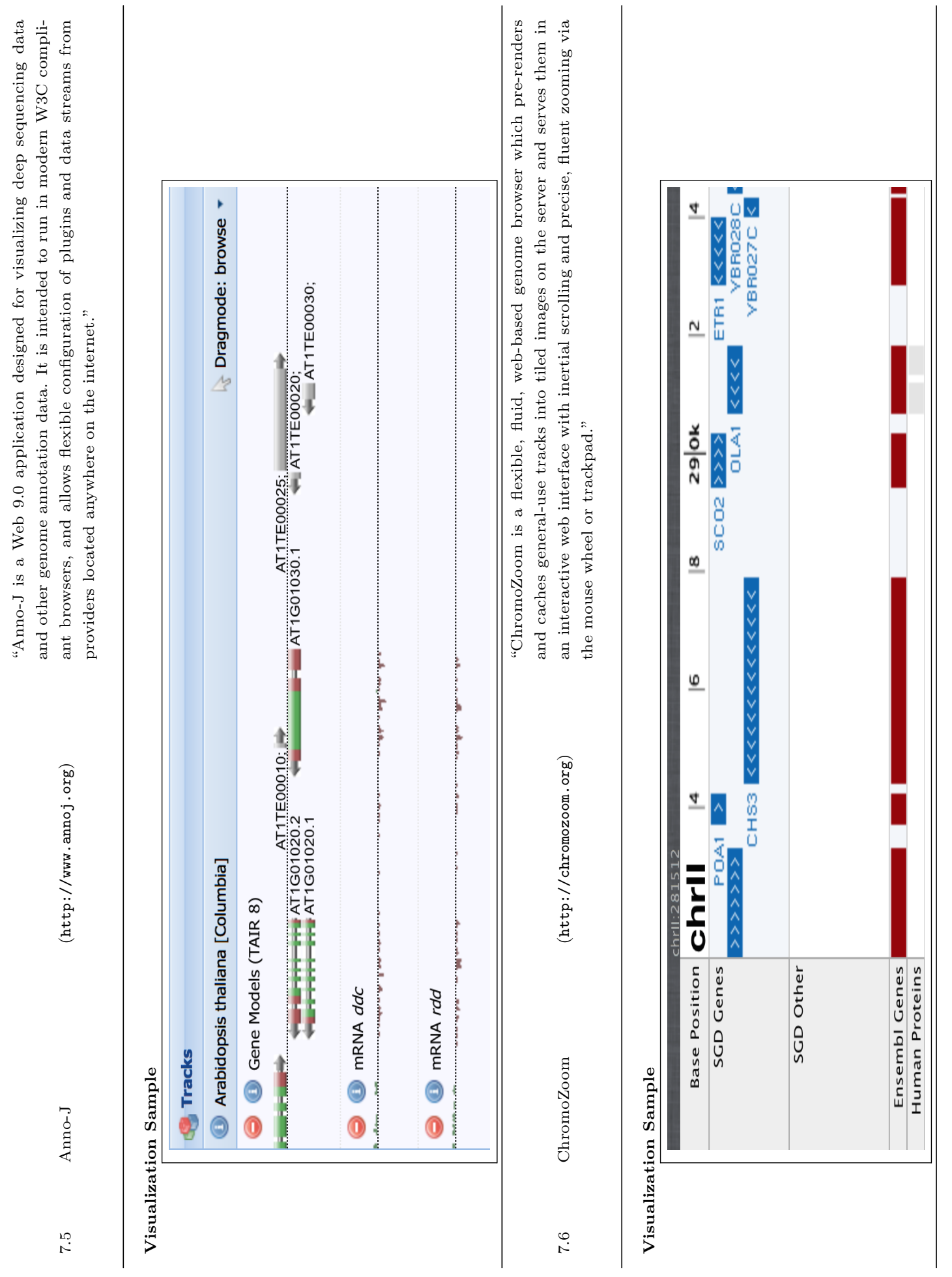



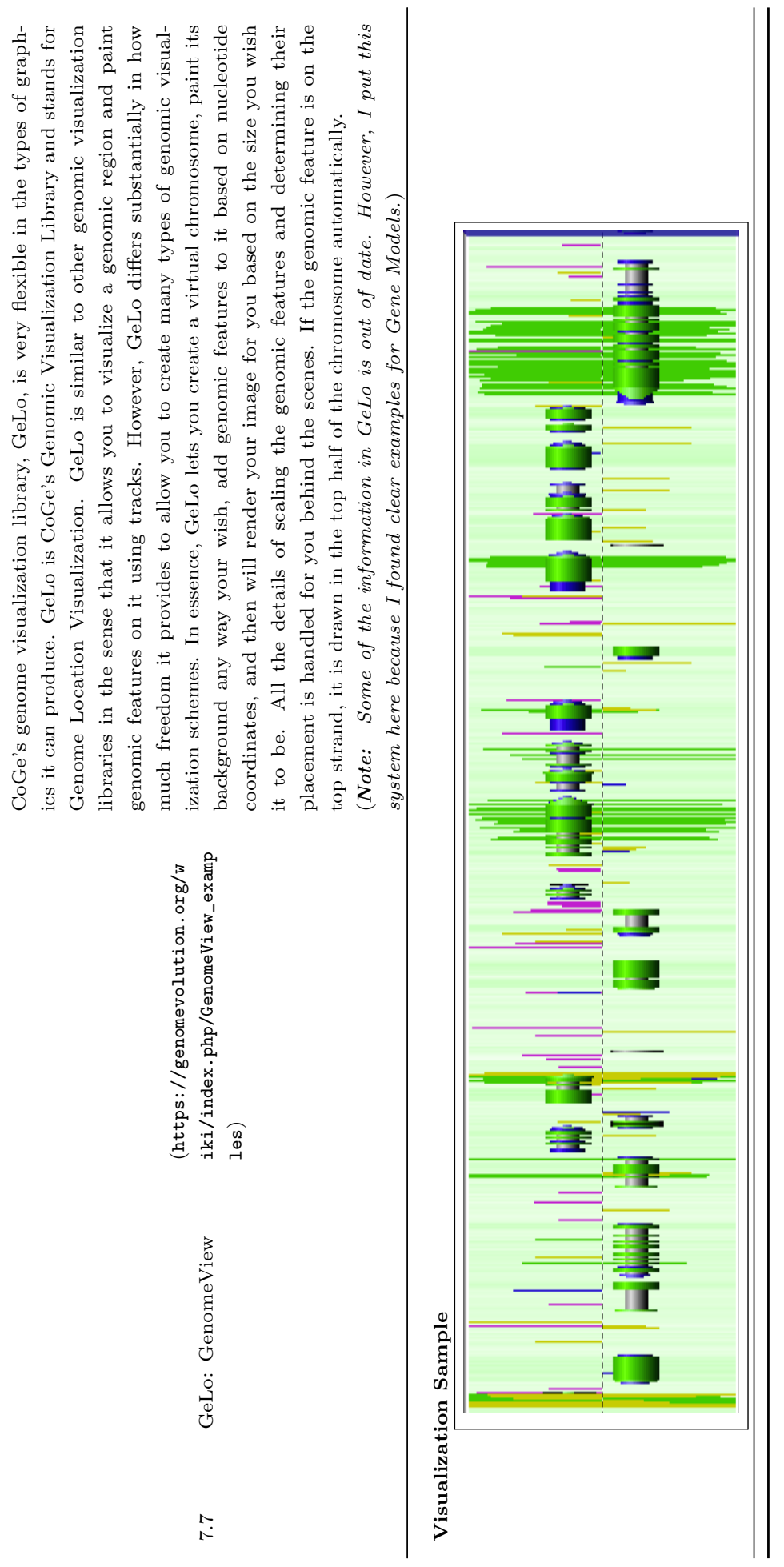


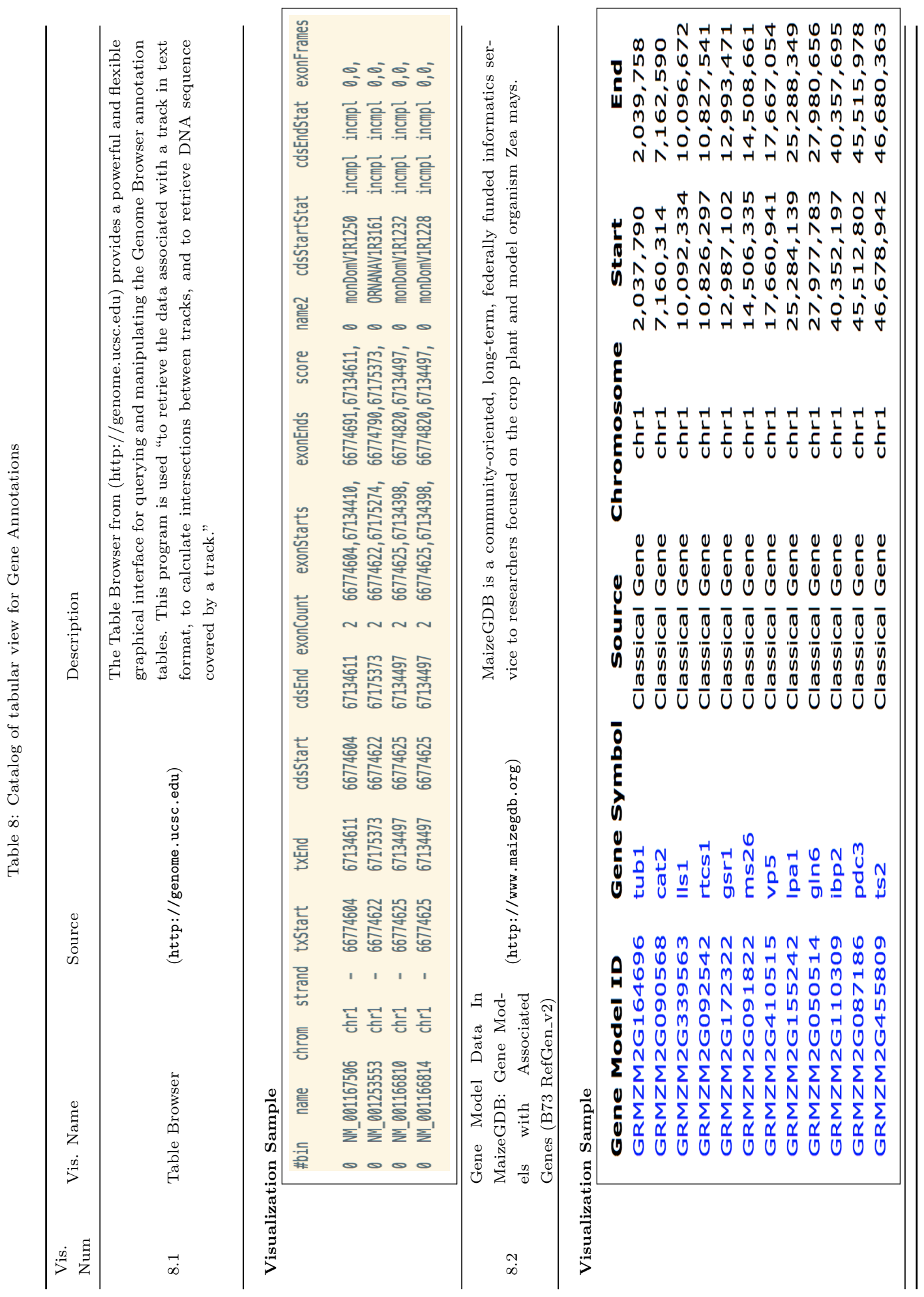


Table 9: Different glyph's visualization for Gene Models

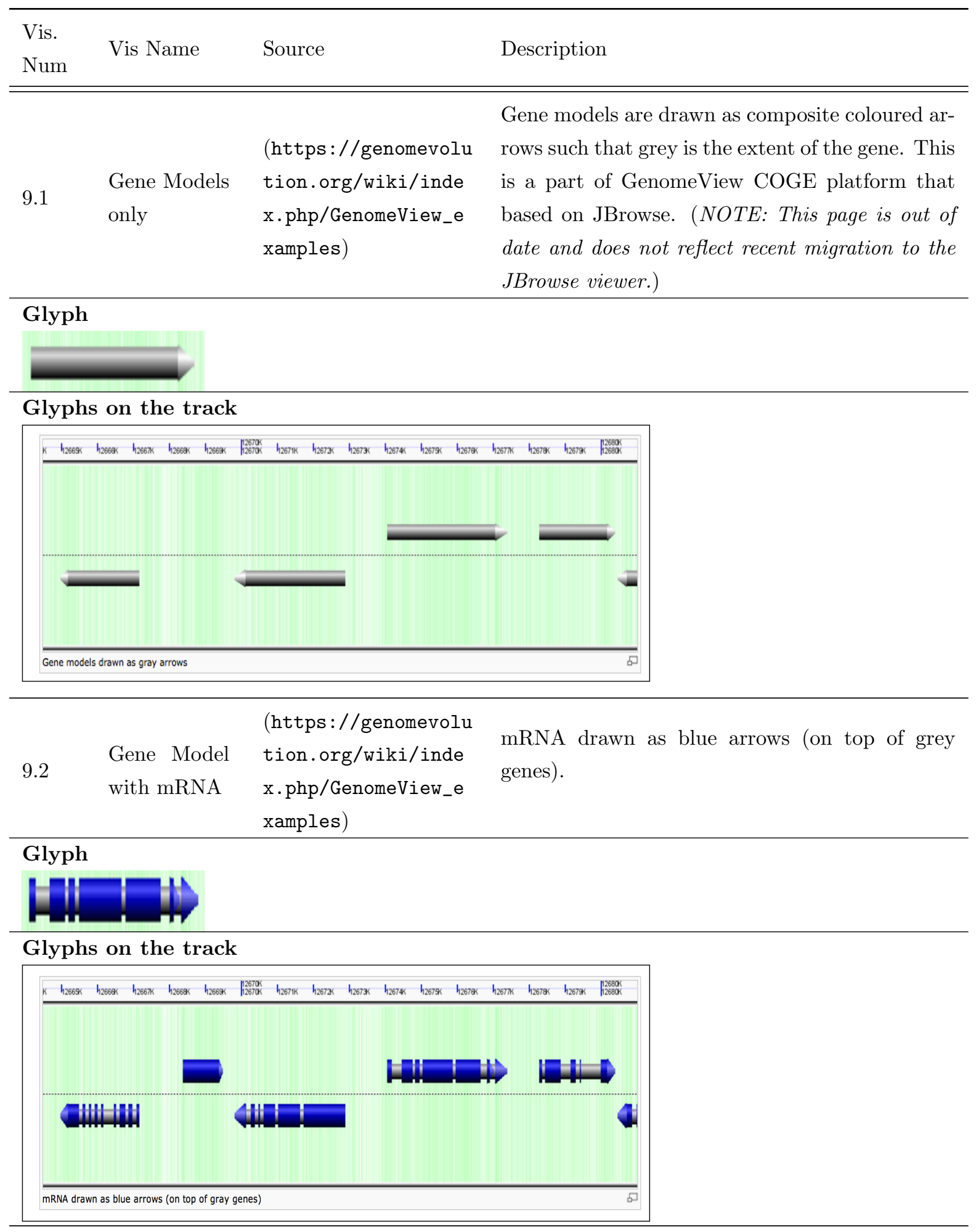




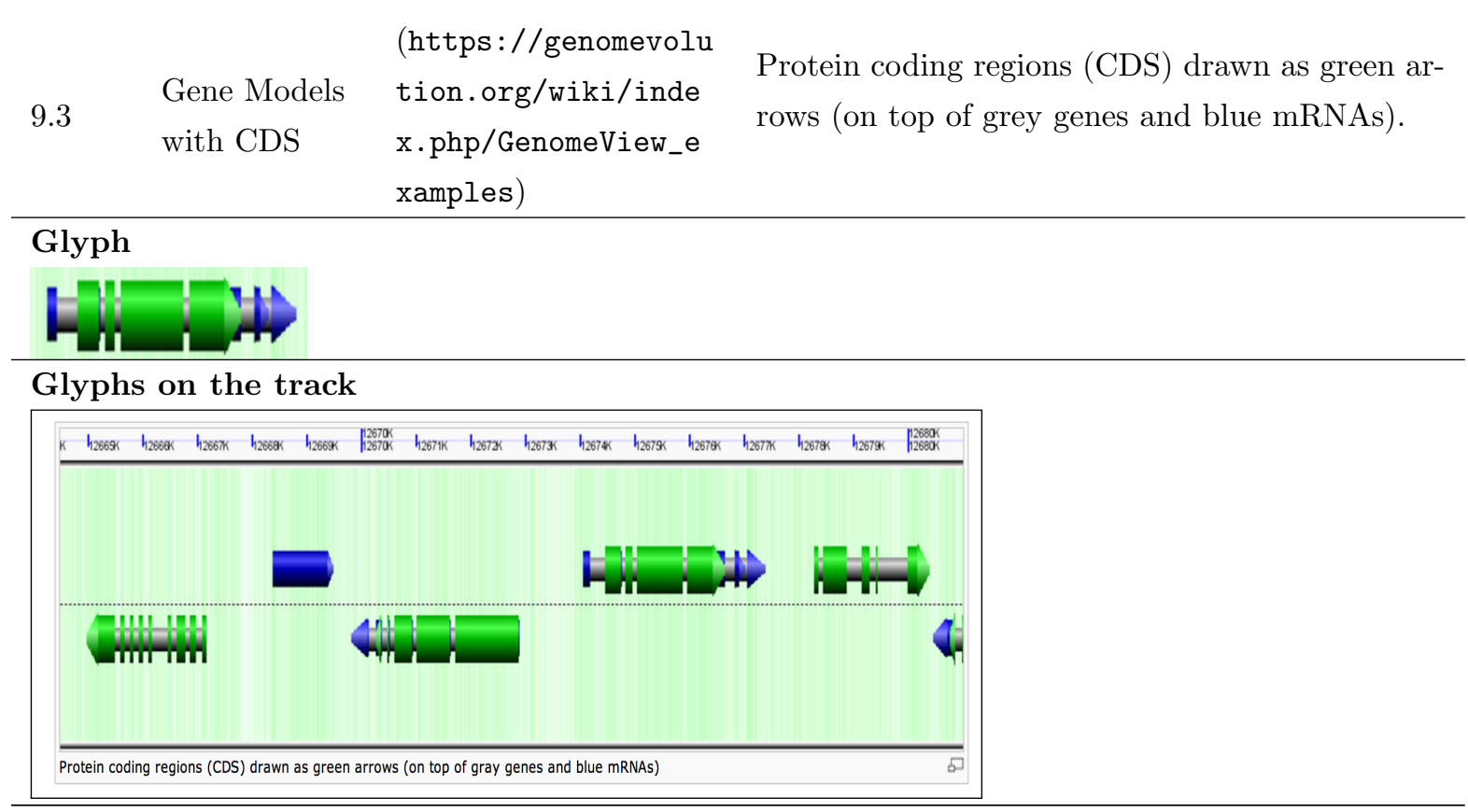

\begin{tabular}{|c|c|c|c|}
\hline \multirow{4}{*}{9.4} & & (http://www.biocat & $\begin{array}{l}\text { A SwitchGear gene model is made up of one or } \\
\text { more cDNAs that align to the same region of the }\end{array}$ \\
\hline & \multirow{3}{*}{$\begin{array}{l}\text { SwitchGear } \\
\text { gene model }\end{array}$} & $. \mathrm{com} / \mathrm{bc} / \mathrm{pdf} / \mathrm{LightS}$ & iuman genome. specincany, swicnuear gen \\
\hline & & witch_Genemodel_Te & moders are aented as ciusters of cDNA allg \\
\hline & & chnote.pdf ) & strand. \\
\hline
\end{tabular}

\section{Glyph}

\section{Gene Model A}

Glyphs on the track

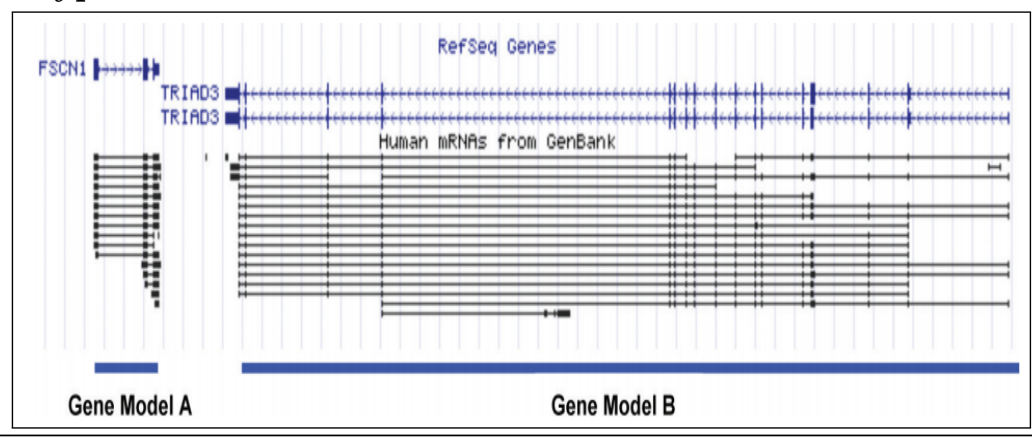

MSU Osa1 Rice Gene Models are gene models an-

$\begin{array}{lll}\text { MSU Osa1 } & \\ 9.5 & \text { Rice Gene } & \text { http://rice.plant } \\ \text { Models } & \text { biology.msu.edu }\end{array}$
notated by the Rice Genome Annotation Project. Gene models are drawn as coloured rectangles. The direction of the transcript is indicated by an arrow attached to the end of the glyph. 


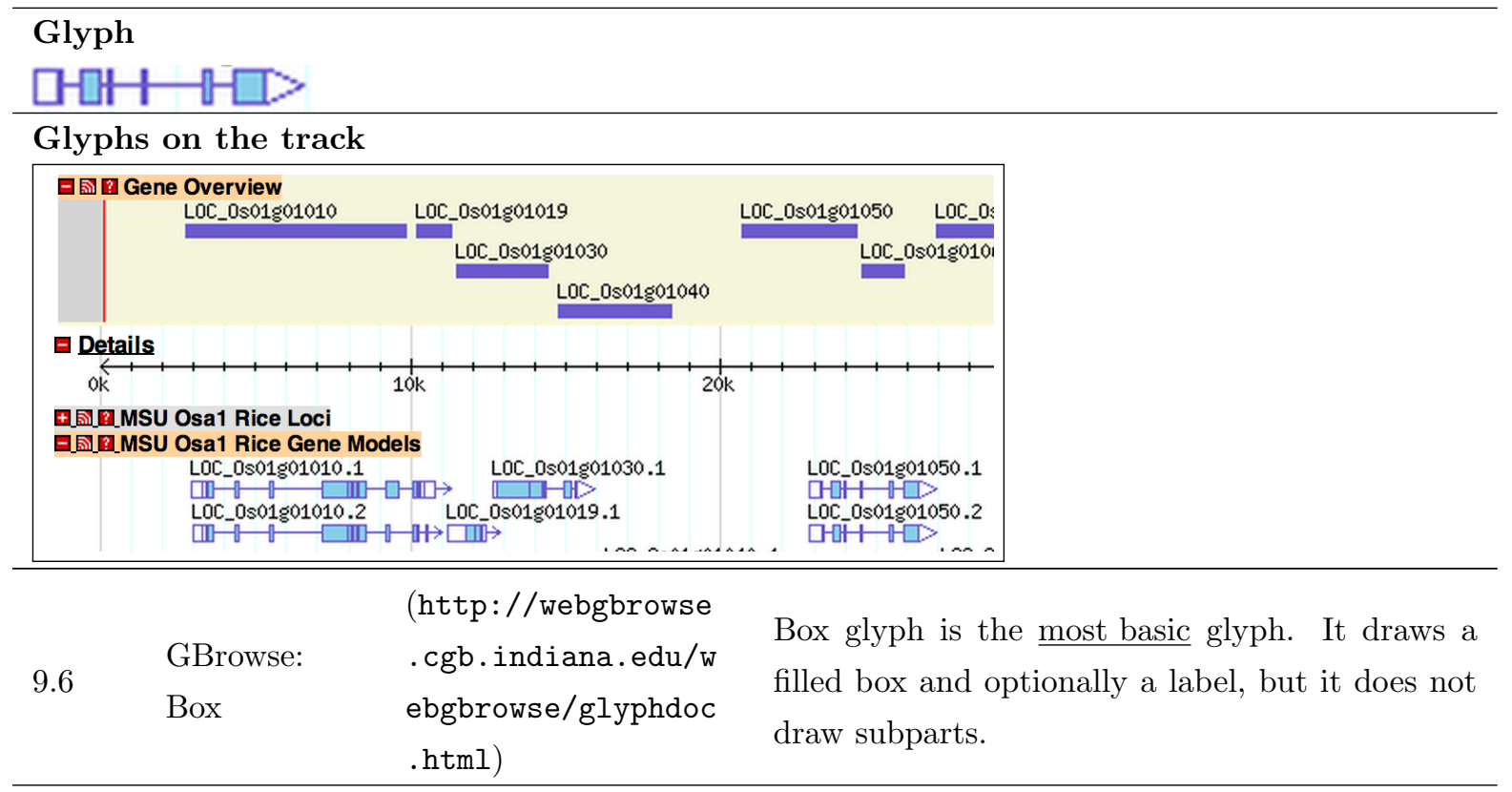

\section{Glyph}

:E: $0 \%$

$\square$

Glyphs on the track

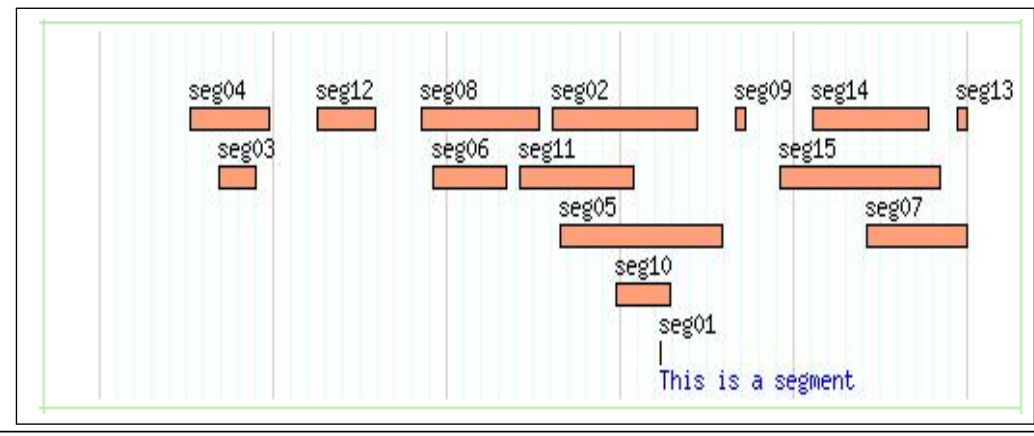

\begin{tabular}{|c|c|c|c|}
\hline \multirow{4}{*}{977} & \multirow{4}{*}{$\begin{array}{l}\text { GBrowse: } \\
\text { Generic }\end{array}$} & (http://webgbrowse & \multirow{4}{*}{$\begin{array}{l}\text { Generic is identical to the box glyph except that } \\
\text { it will draw the subparts of features that contain } \\
\text { subfeatures. Generic is the default glyph used in } \\
\text { GBrowse. }\end{array}$} \\
\hline & & .cgb.indiana.edu/w & \\
\hline & & ebgbrowse/glyphdoc & \\
\hline & & .html) & \\
\hline
\end{tabular}

\section{Glyph}

sego6

ᄃ-D-- $\mathrm{C}$

Glyphs on the track 


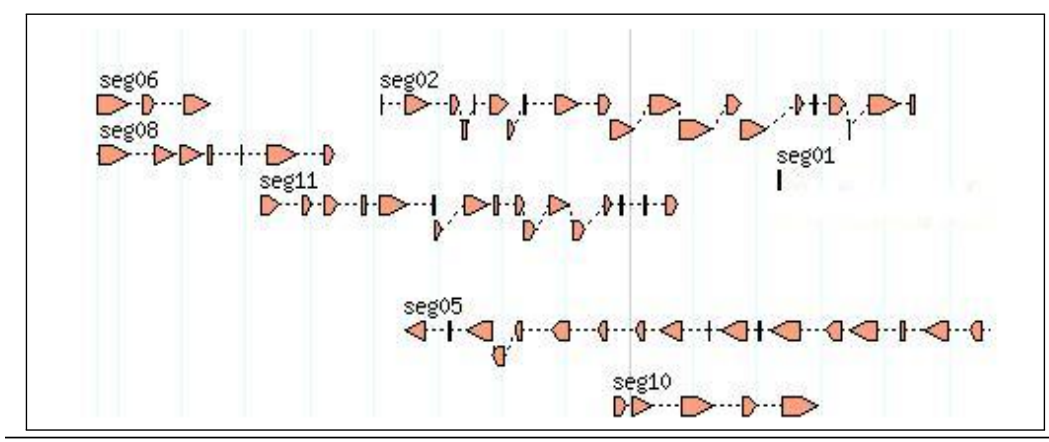

Cds glyph draws features associated with a pro-

\begin{tabular}{|c|c|c|c|}
\hline \multirow{4}{*}{9.8} & \multirow{4}{*}{$\begin{array}{l}\text { GBrowse: } \\
\text { CDS }\end{array}$} & (http://webgbrowse & \multirow{4}{*}{$\begin{array}{l}\text { tein coding region. A series of color-coded boxes } \\
\text { indicating the translation frame are drawn at high } \\
\text { magnifications. But at low magnifications, the } \\
\text { amino acid sequence of the resulting protein is } \\
\text { drawn. }\end{array}$} \\
\hline & & .cgb.indiana.edu/w & \\
\hline & & ebgbrowse/glyphdoc & \\
\hline & & .html) & \\
\hline
\end{tabular}

Glyph
Apple1

\section{Glyphs on the track}

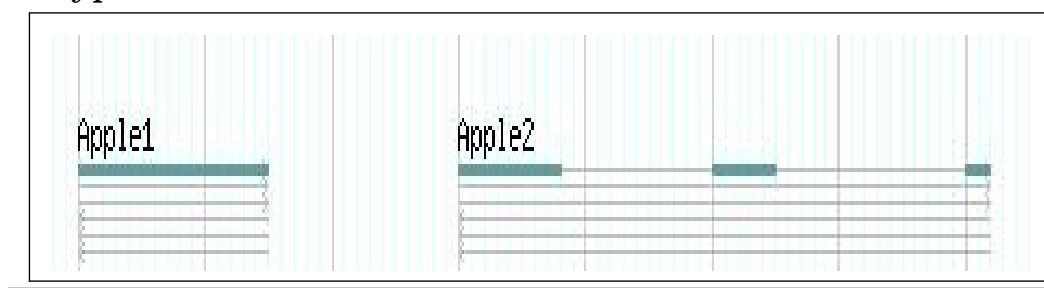

The gene glyph is used for drawing genes that may have alternatively-spliced transcripts. (http://webgbrowse The various isoforms are stacked on top of each ebgbrowse/glyphdoc .html) other and given a single label and description that apply to the entire stack. The name of each individual transcript is optionally printed to the left of the transcript glyph.

\section{Glyph}

EDEN.14

Glyphs on the track 


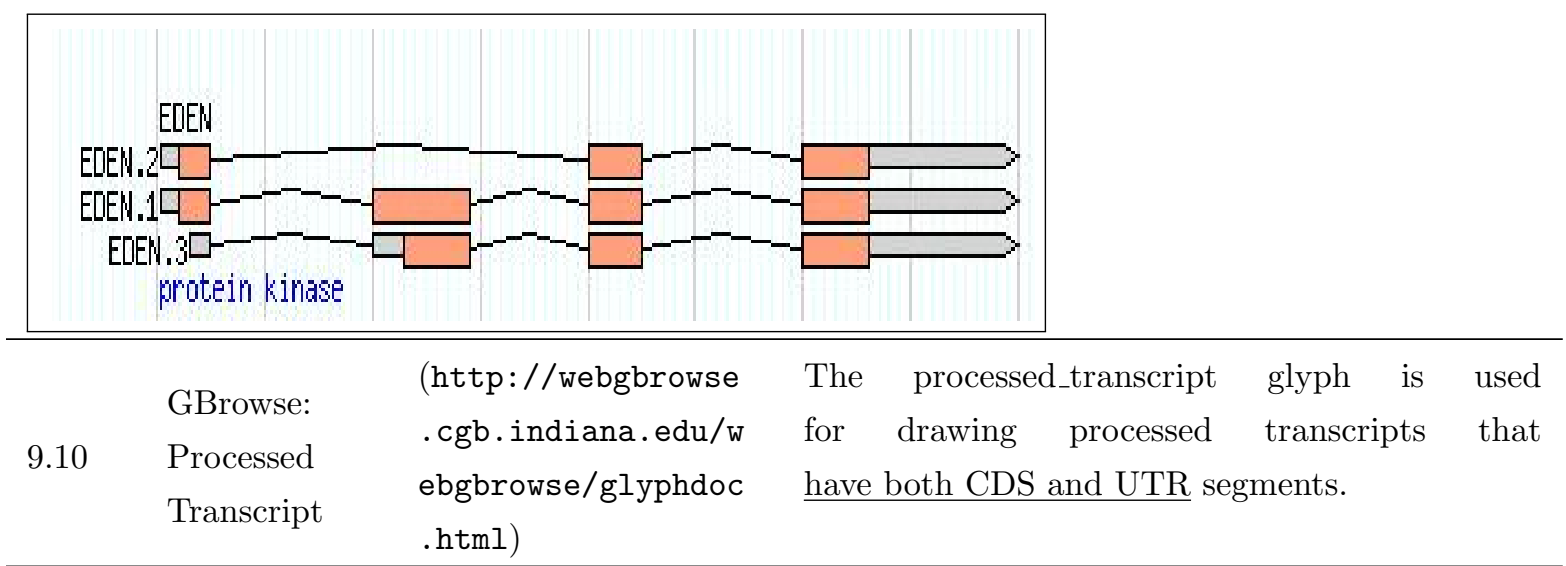

\section{Glyph \\ EDEN.1 \\ 따 $\square$ \\ Eden splice form 1}

\section{Glyphs on the track}

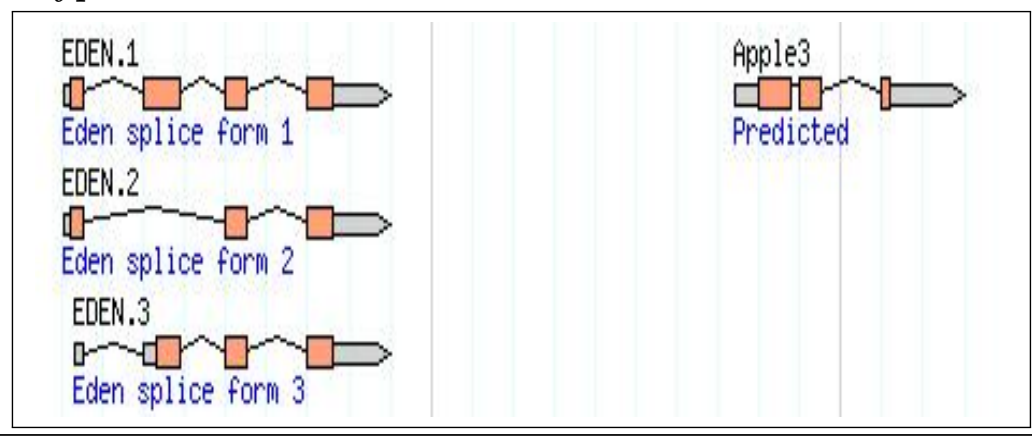

The Transcript glyph is used for drawing tran-

(http://webgbrowse

$9.11 \quad \begin{aligned} & \text { GBrowse: } \\ & \text { Transcript }\end{aligned}$ .cgb. indiana. edu/w ebgbrowse/glyphdoc

.html scripts. It is essentially a segments glyph in which the default connecting segments are hats. The direction of the transcript is indicated by an arrow attached to the end of the glyph. 
The Transcript2 glyph is used for draw-

(http://webgbrowse

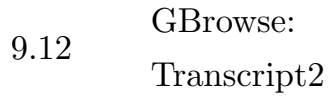

. cgb. indiana. edu/w

ebgbrowse/glyphdoc

.html) ing transcripts. It is like transcript except that if there is sufficient room the terminal exon is shaped like an arrow in order to indicate the direction of transcription. If there is not enough room, a small arrow is drawn.

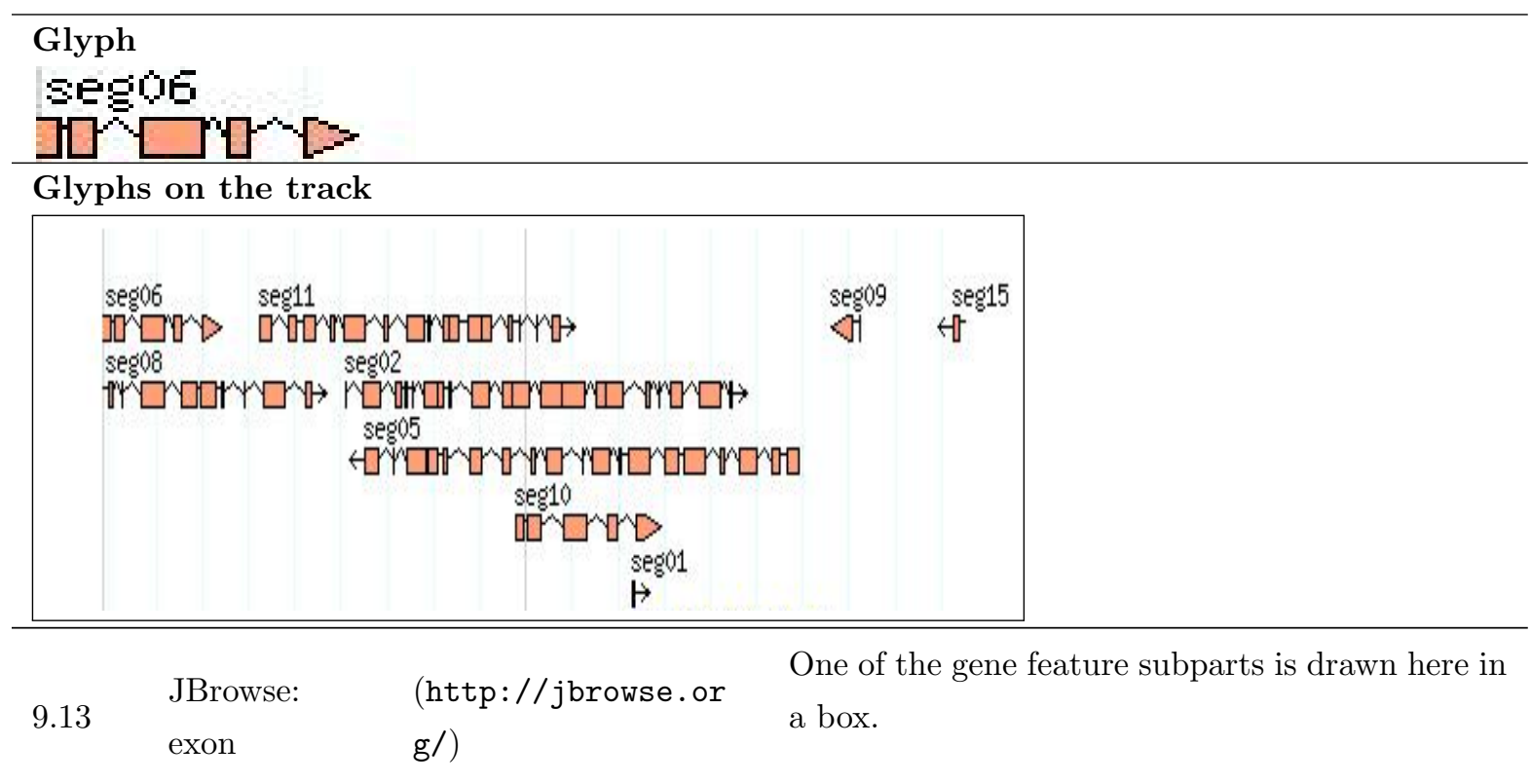

\section{Glyph}

\section{Glyphs on the track}

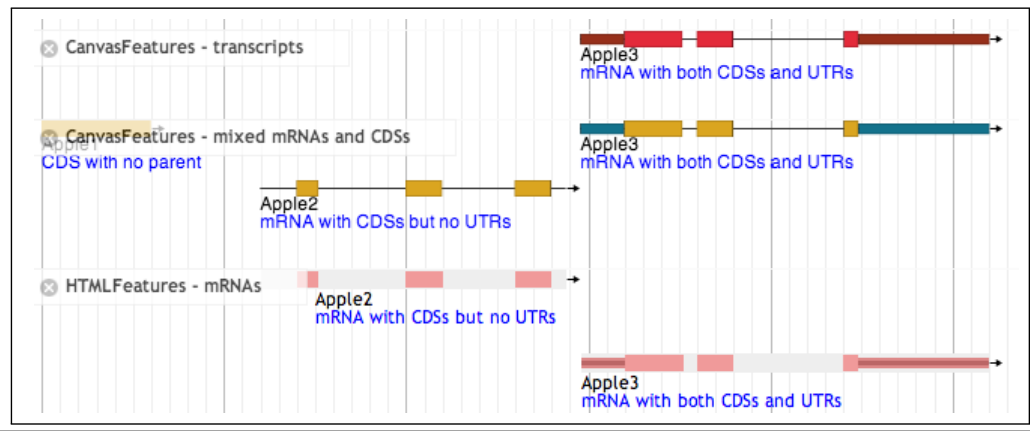


JBrowse:

9.14 mRNA with $\begin{aligned} & \text { http://jbrowse.or } \\ & \text { CDs but no g// } \\ & \text { UTRs }\end{aligned}$

This glyph draws features that are associated with a protein coding region. At high magnifications, it draws a series of boxes that are colorcoded to indicate the frame in which the translation occurs. At low magnifications, it draws the amino acid sequence of the resulting protein. Amino acids that are created by a splice are optionally shown in a distinctive color. Credit: http://search.cpan.org/ lds/Bio-Graphic s-2.39/lib/Bio/Graphics/Glyph/cds.pm

\section{Glyph}

\section{Glyphs on the track}

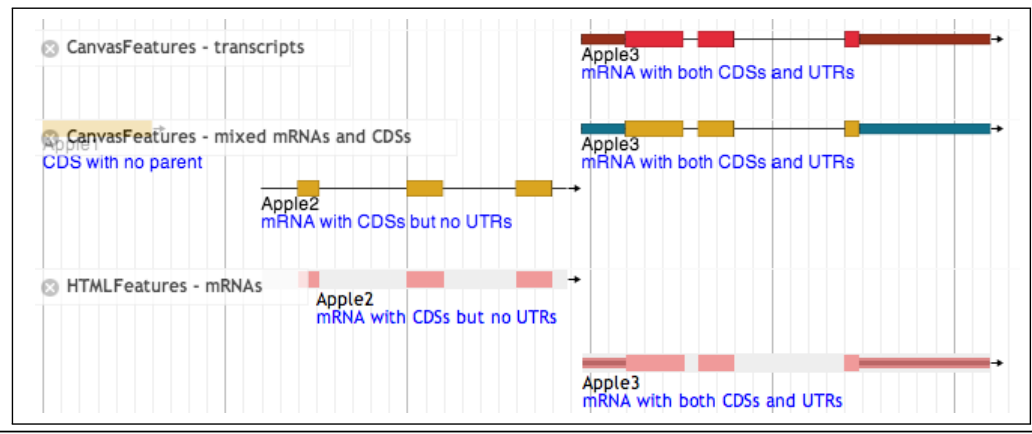


Appendix B

\section{Protein Domain Catalog}




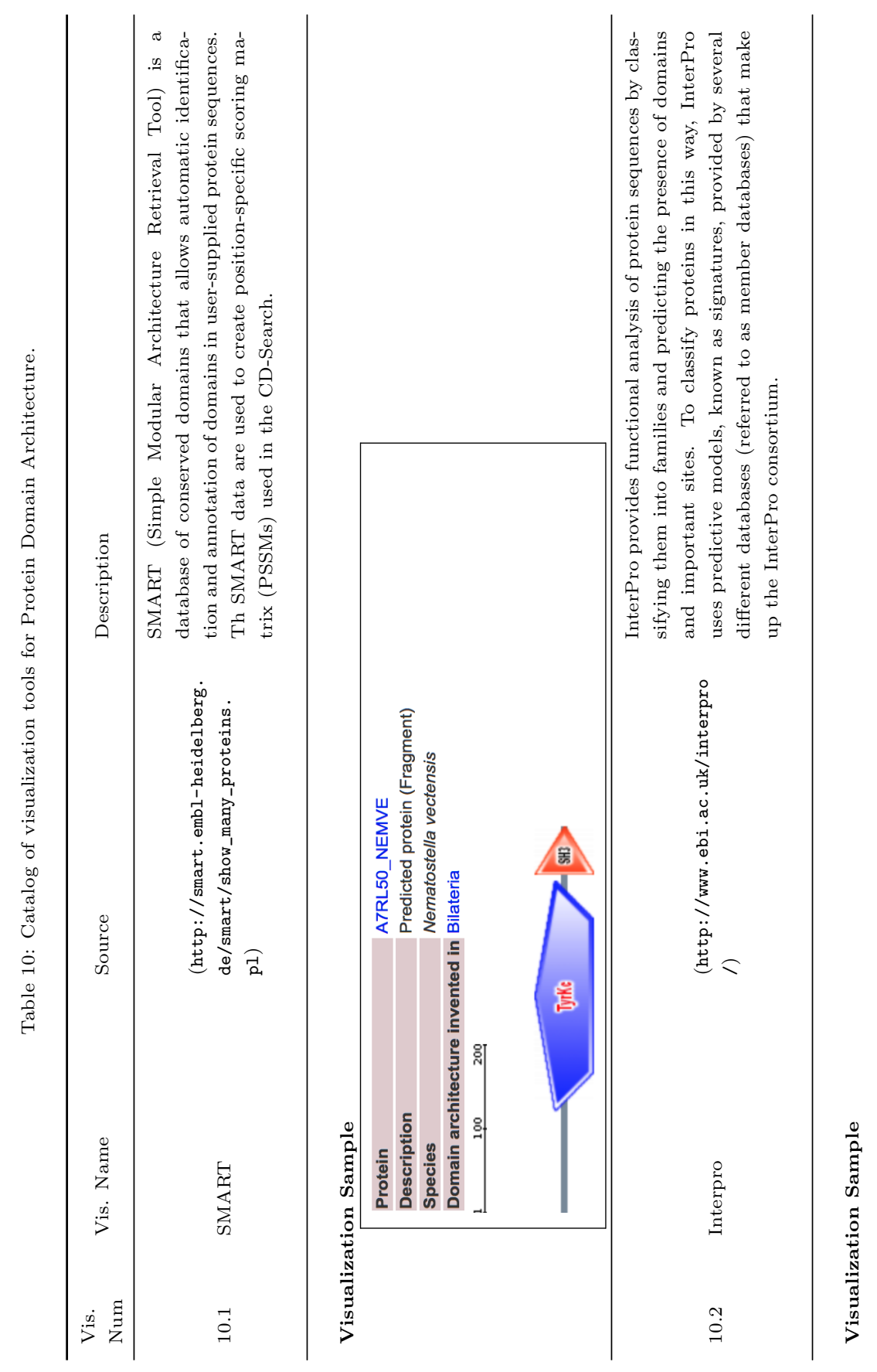




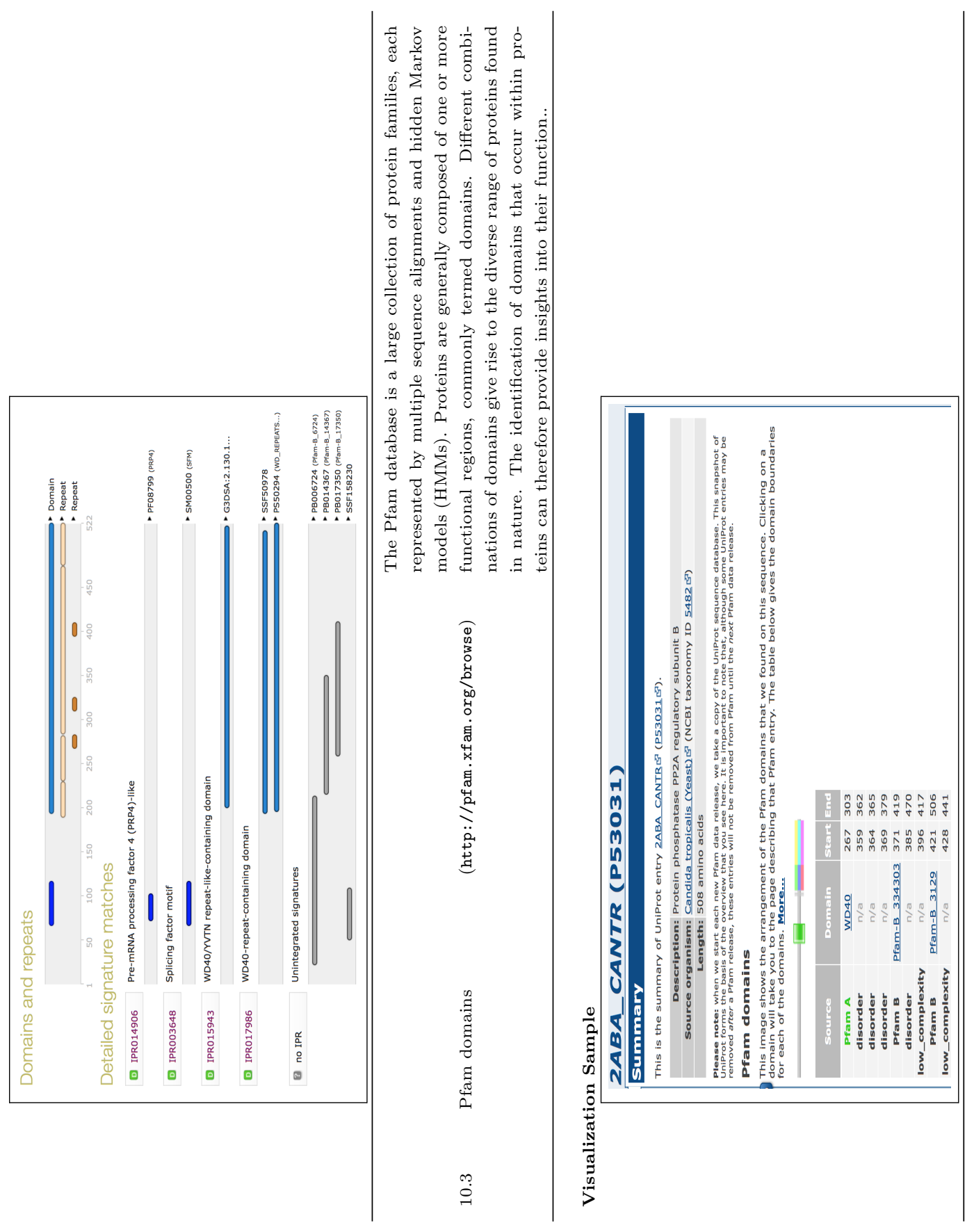




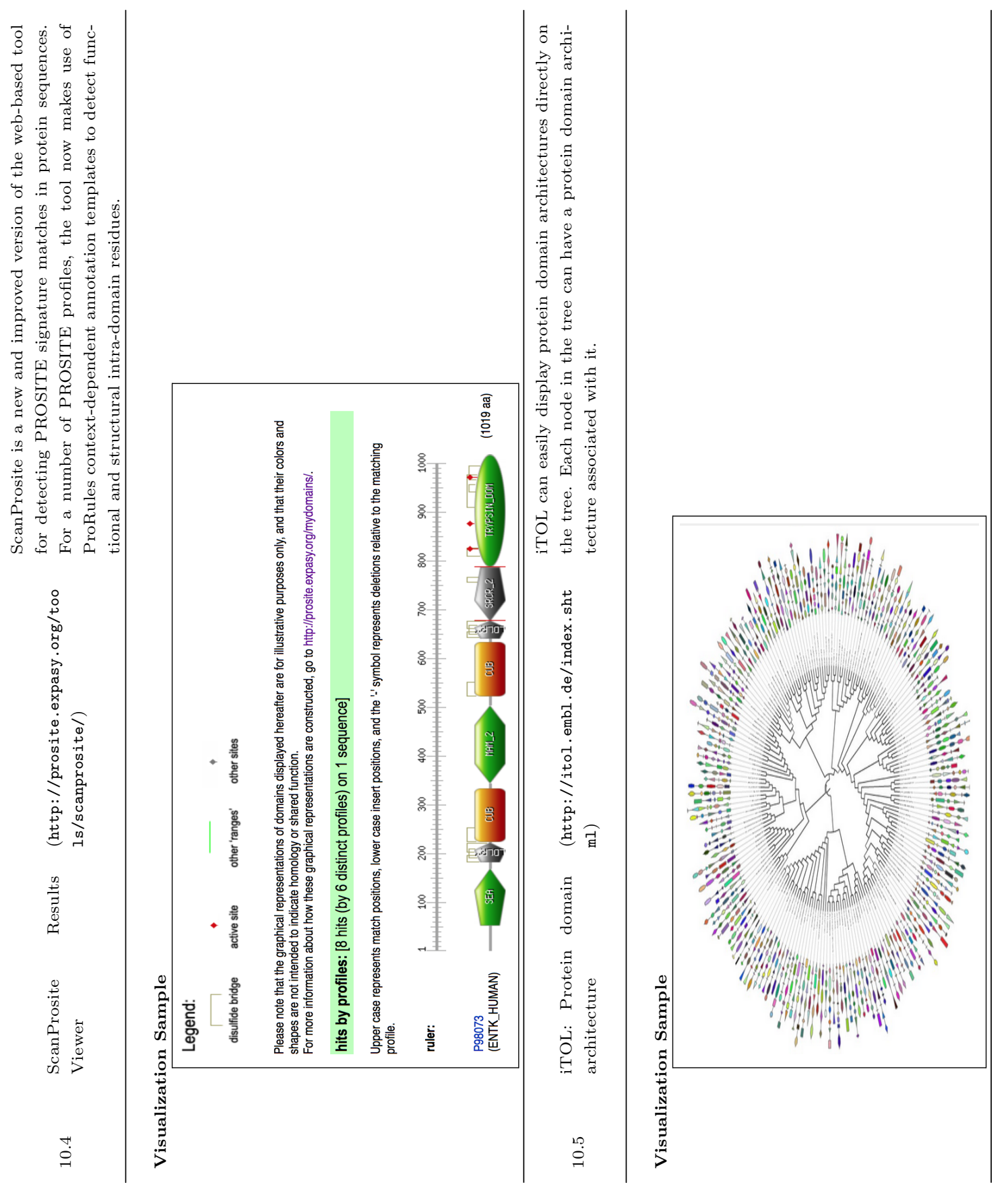




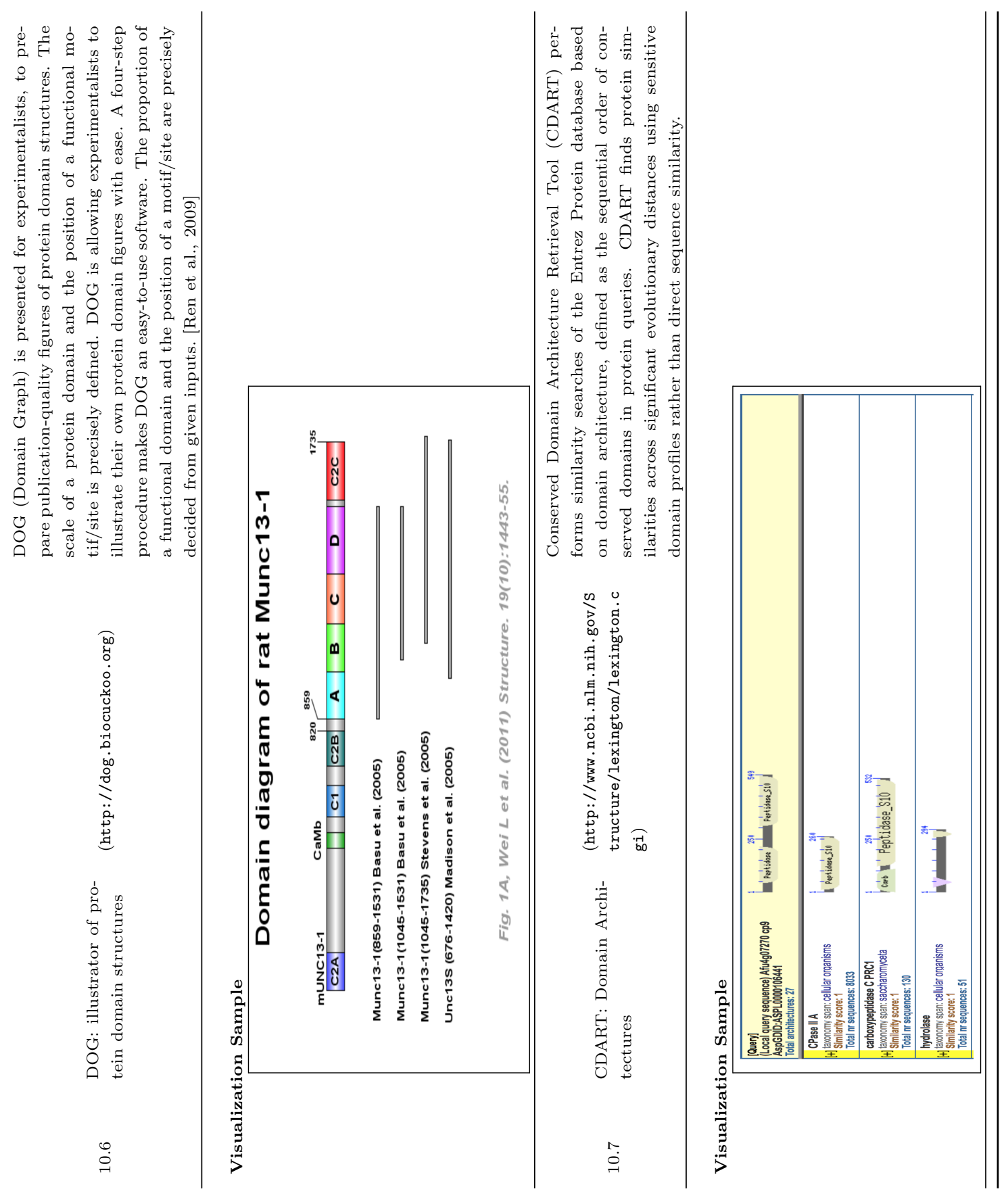


Table 11: Different glyph's for Protein Domain Architecture

\begin{tabular}{|c|c|c|c|}
\hline $\begin{array}{l}\text { Vis. } \\
\text { Num }\end{array}$ & Vis Name & Source & Description \\
\hline 111 & $\begin{array}{l}\text { iTOL: Pro- } \\
\text { tein domain } \\
\text { architecture }\end{array}$ & $\begin{array}{l}\text { Letunic and Bork, } \\
2007\end{array}$ & $\begin{array}{l}\text { Each node in the tree can have a protein domain } \\
\text { architecture associated with it. Even though its pri- } \\
\text { mary use is for the display of protein domains, it can } \\
\text { be used for various other purposes. The format is } \\
\text { as follows: Each line should have a node ID, total } \\
\text { protein length, and the definitions of the domains. } \\
\text { The domain definitions field contains one or more } \\
\text { domains, separated using the same character which } \\
\text { is used in the first two fields. Each domain defini- } \\
\text { tion consists of } 5 \text { parts, separated with vertical lines } \\
\text { (|). For example: (RE |100|150|\#ff0000|SH2). The } \\
\text { fields are: } \\
\text { 1. RE: } 2 \text { character code defining the domain } \\
\text { shape (see below for supported shapes) } \\
\text { 2. 100: domain start position } \\
\text { 3. 150: domain end position } \\
\text { 4. \#ff0000: color definition (hexadecimal RGB } \\
\text { notation) } \\
\text { 5. SH2: domain label. } \\
\text { shown in interactive mode, since you can hover your } \\
\text { mouse cursor over any domain to show its label and } \\
\text { other associated information. Domain architectures } \\
\text { cor internal nodes will only be displayed on collapsed } \\
\text { - shtml. }\end{array}$ \\
\hline
\end{tabular}




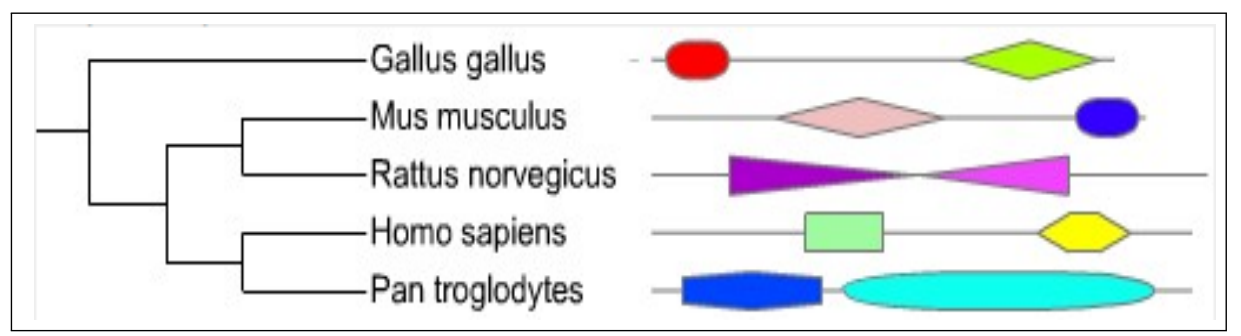

\section{supported shapes}

\begin{tabular}{cc}
\hline Code & Shape \\
$\mathrm{RE}$ & rectangle \\
\hline $\mathrm{HH}$ & horizontal hexagon \\
\hline $\mathrm{HV}$ & vertical hexagon \\
\hline $\mathrm{EL}$ & ellipse \\
\hline $\mathrm{DI}$ & rhombus (diamond) \\
\hline $\mathrm{TR}$ & right pointing triangle \\
\hline $\mathrm{TL}$ & left pointing triangle \\
\hline $\mathrm{PL}$ & left pointing pentagram \\
\hline $\mathrm{PR}$ & right pointing pentagram \\
\hline $\mathrm{PU}$ & up pointing pentagram \\
\hline $\mathrm{PD}$ & down pointing pentagram \\
\hline $\mathrm{OC}$ & octagon \\
\hline $\mathrm{GP}$ & rectangle (gap) \\
\hline
\end{tabular}

\begin{tabular}{|l|l|l|l|l}
\hline 1125 & $\begin{array}{l}\text { SMART: do- } \\
\text { main architec- } \\
\text { ture }\end{array}$ & $\begin{array}{l}\text { "SMART (a Simple Modular Architecture Research } \\
\text { Tool) allows the identification and annotation of ge- } \\
\text { netically mobile domains and the analysis of domain } \\
\text { architectures". Green/grey lines indicate predicted } \\
\text { coiled coil regions and pink lines show low complex- } \\
\text { ity segments. Regions of proteins without any pre- } \\
\text { dicted features are marked with grey bars and can } \\
\text { be subjected individually to gapped BLAST searches } \\
\text { Schultz et al., 2000. }\end{array}$ \\
\hline
\end{tabular}

\section{Glyphs}

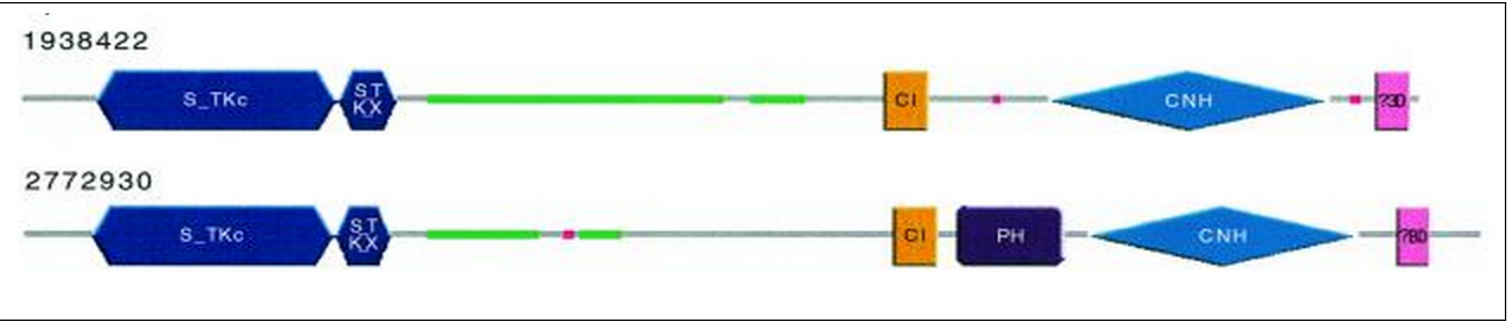




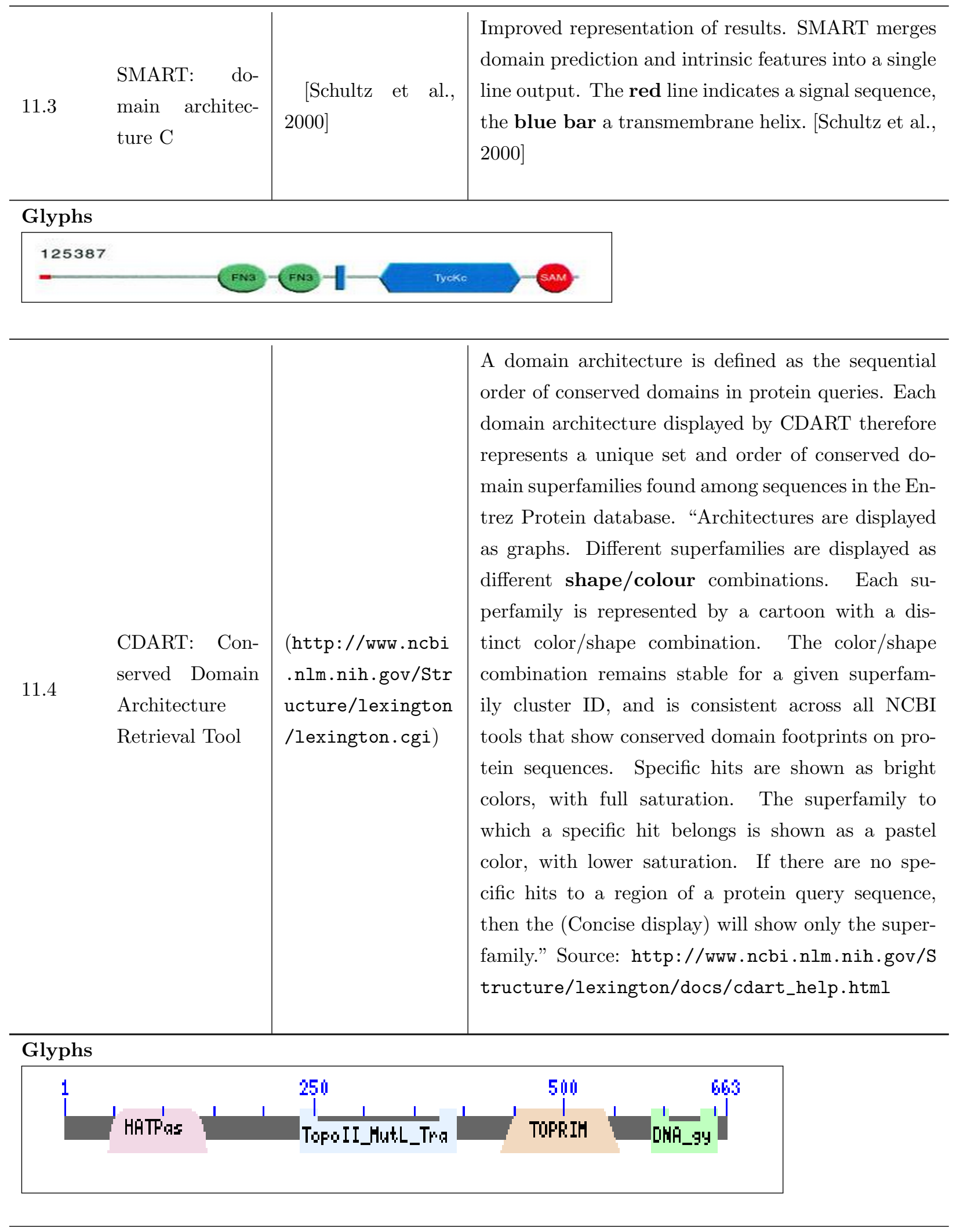


Example of cartoons used to depict conserver domains

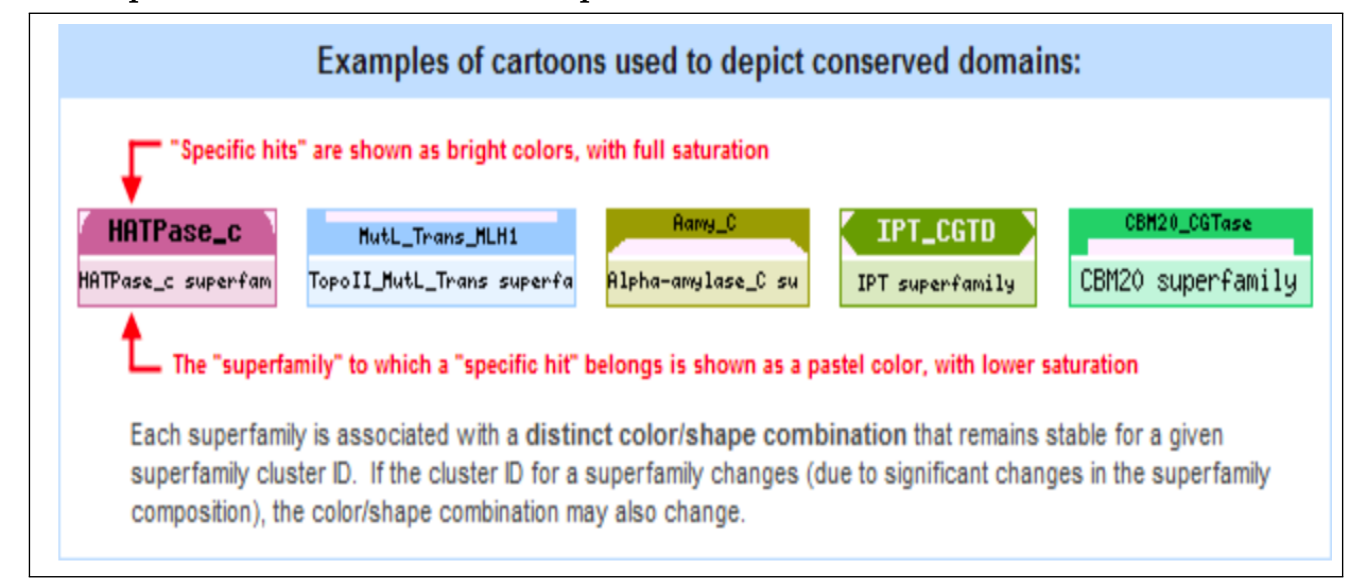

\begin{tabular}{l|l|l}
\hline 115 DOG: Domain & $\begin{array}{l}\text { DOG is allowing experimentalists to illustrate their } \\
\text { own protein domain figures with ease.The proportion } \\
\text { of a functional domain and the position of a mo- } \\
\text { tif/site are precisely decided from given inputs } \\
\text { et al., 2009. The labels can be written in the top or }\end{array}$ \\
$\begin{array}{l}\text { in the bottom depending on the user choice. } \\
\text { Ren et al., 2009 }\end{array}$ \\
\hline
\end{tabular}

\section{Glyphs}

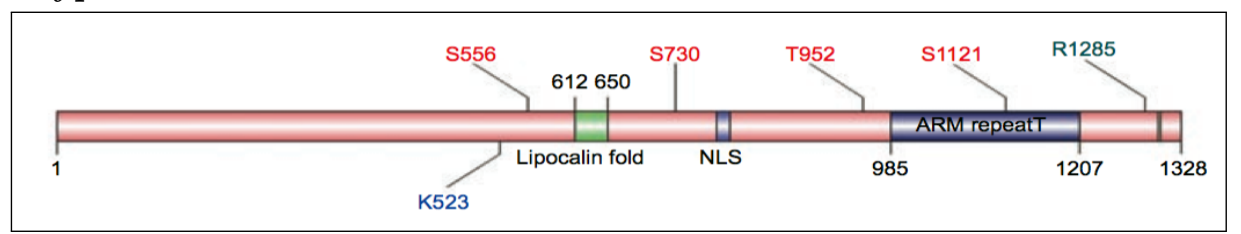




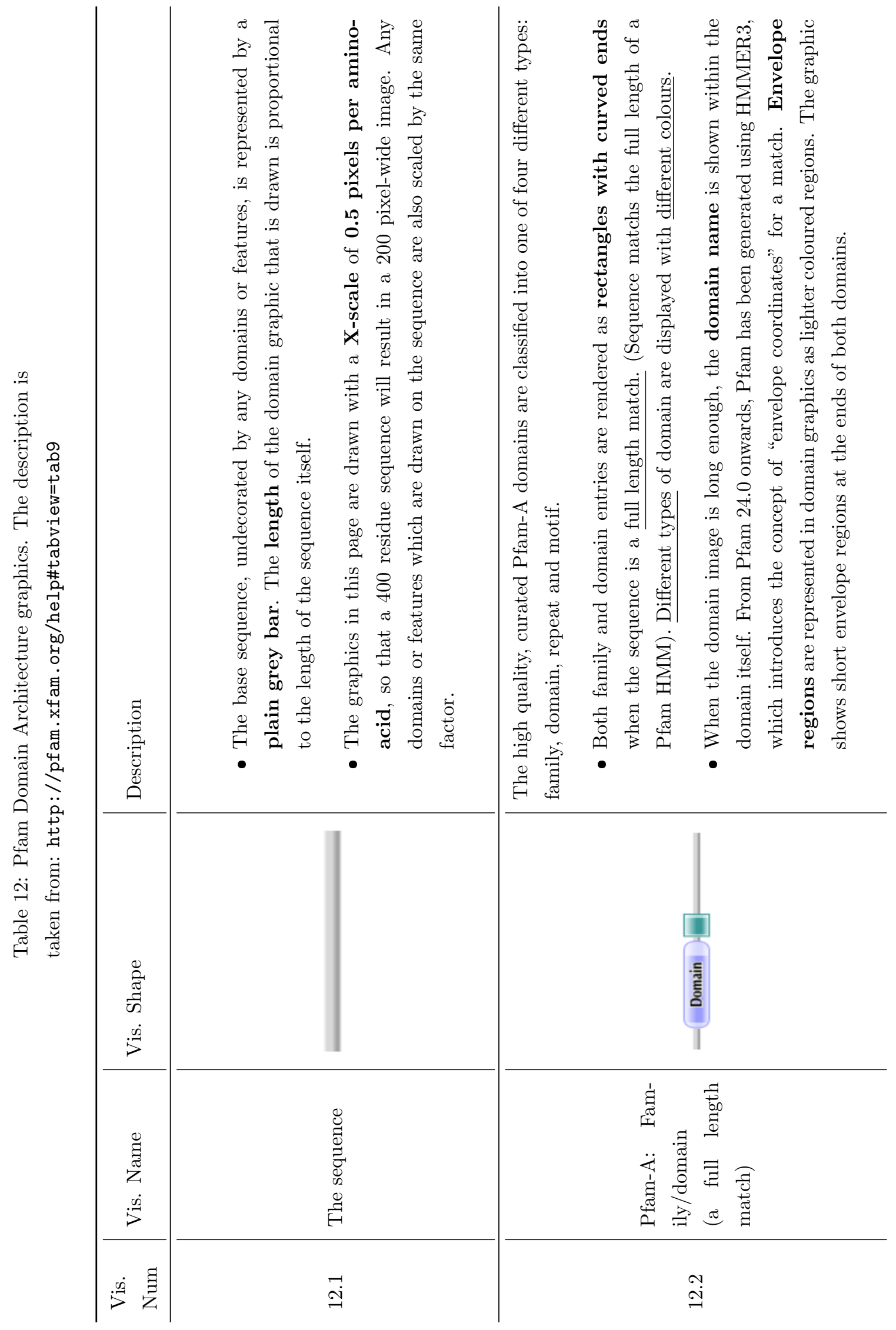




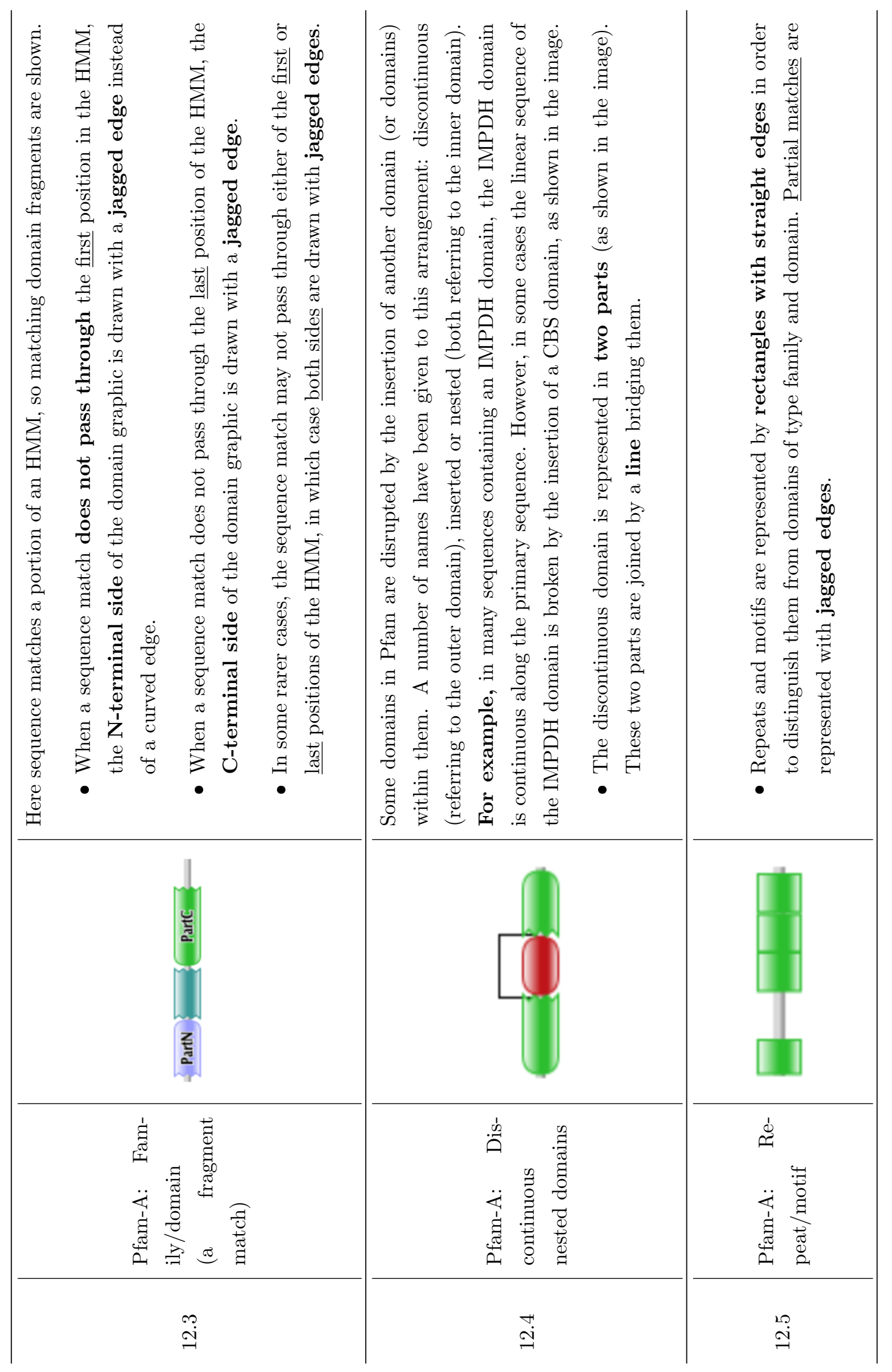




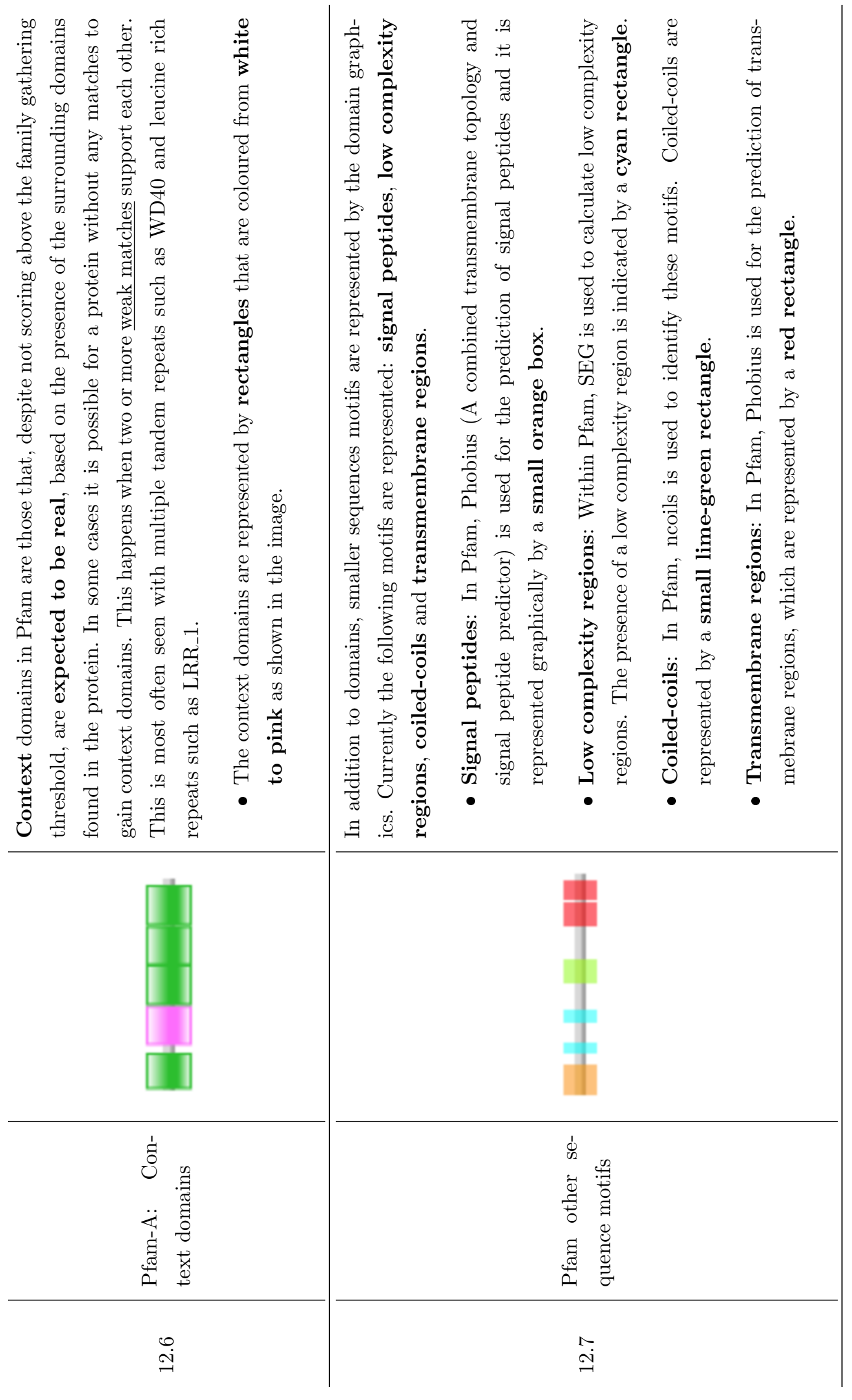




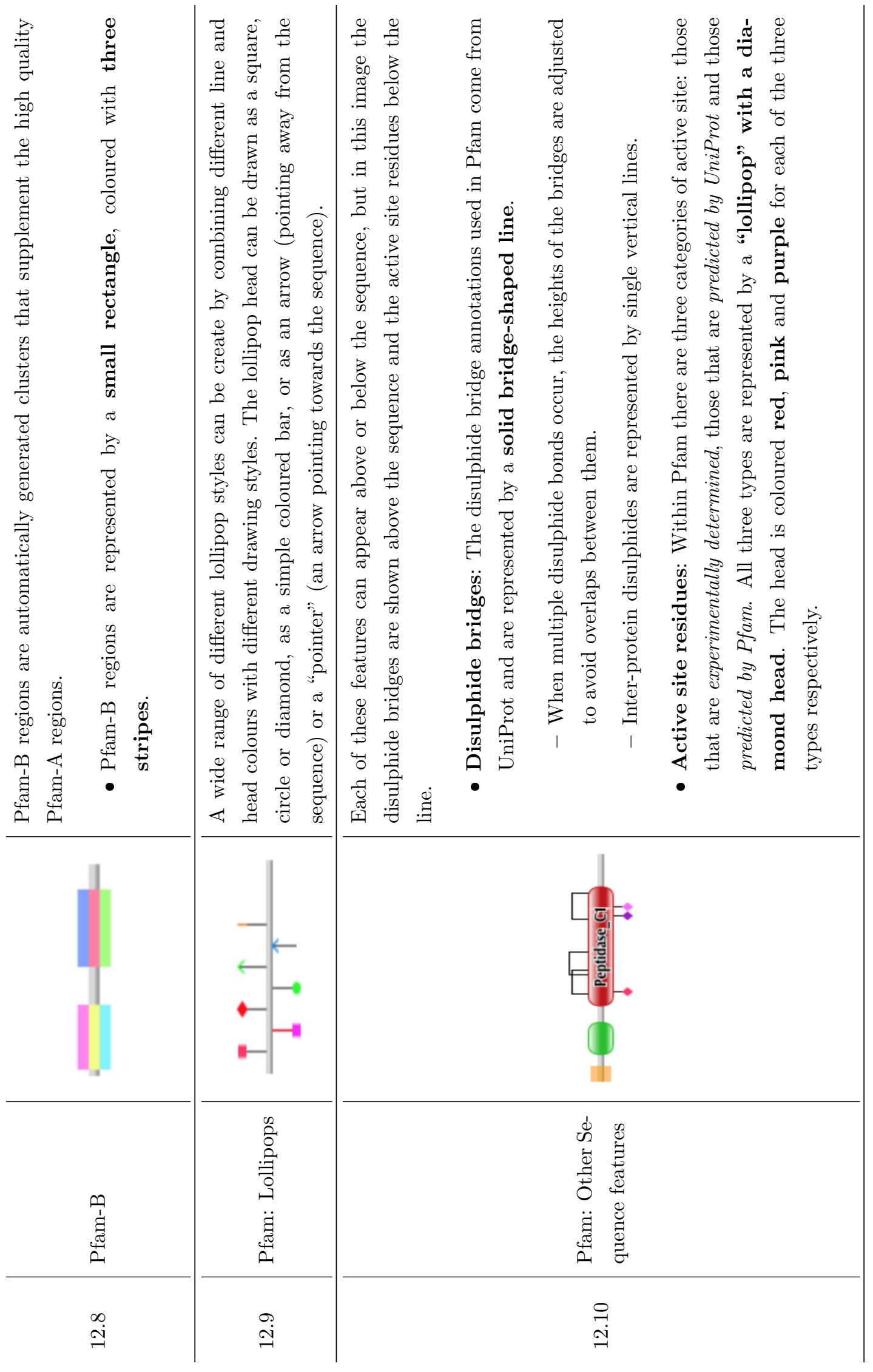




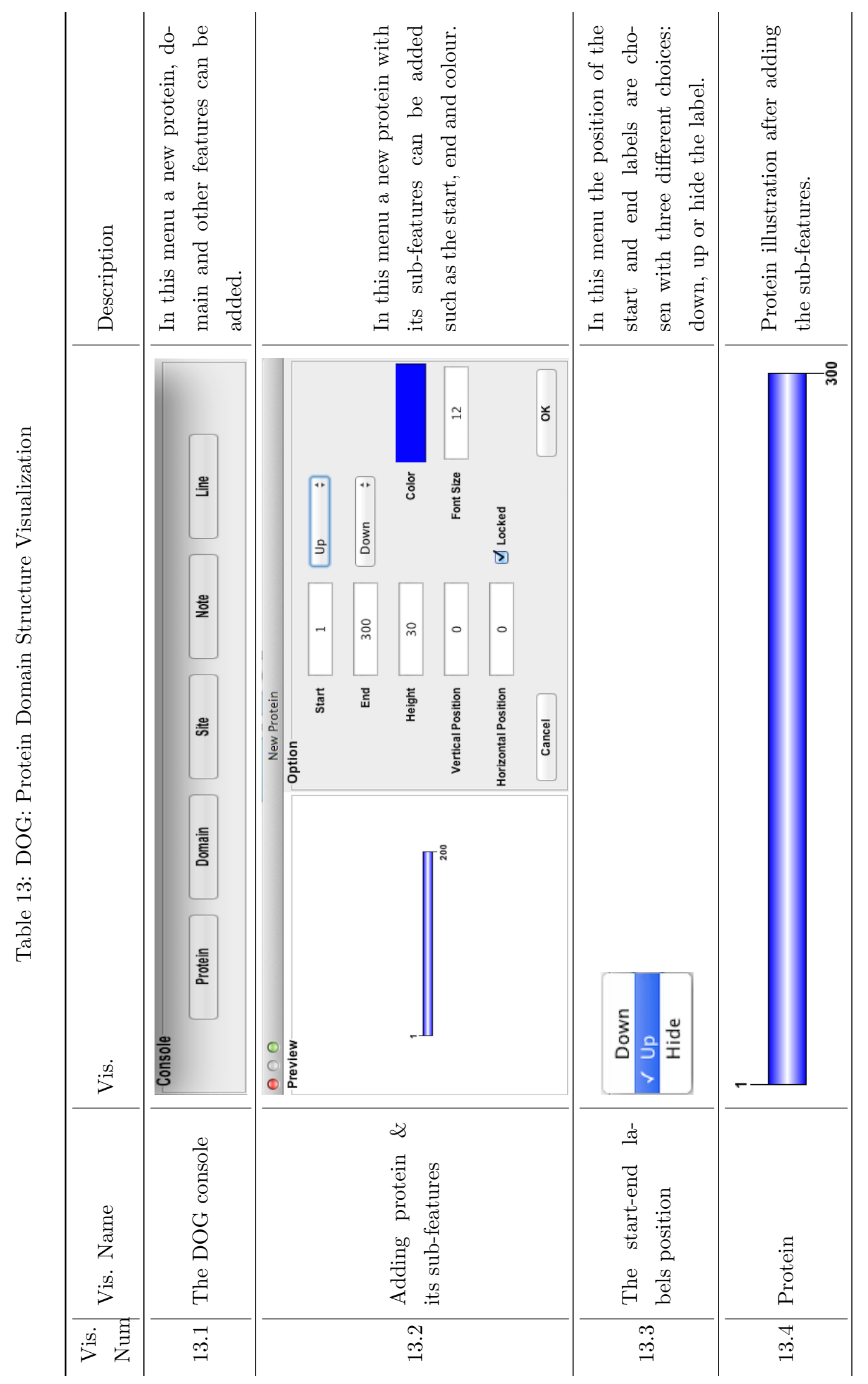




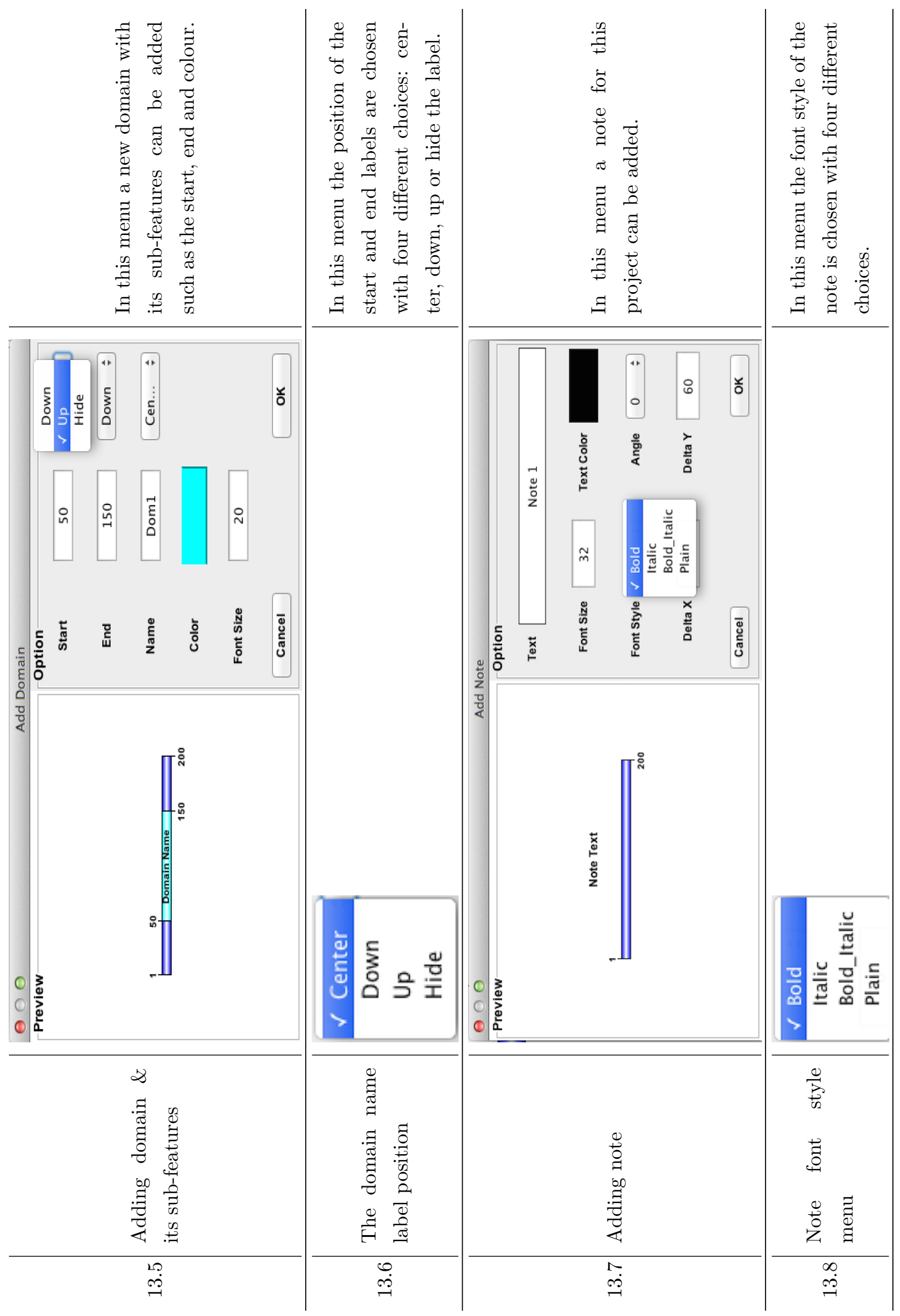




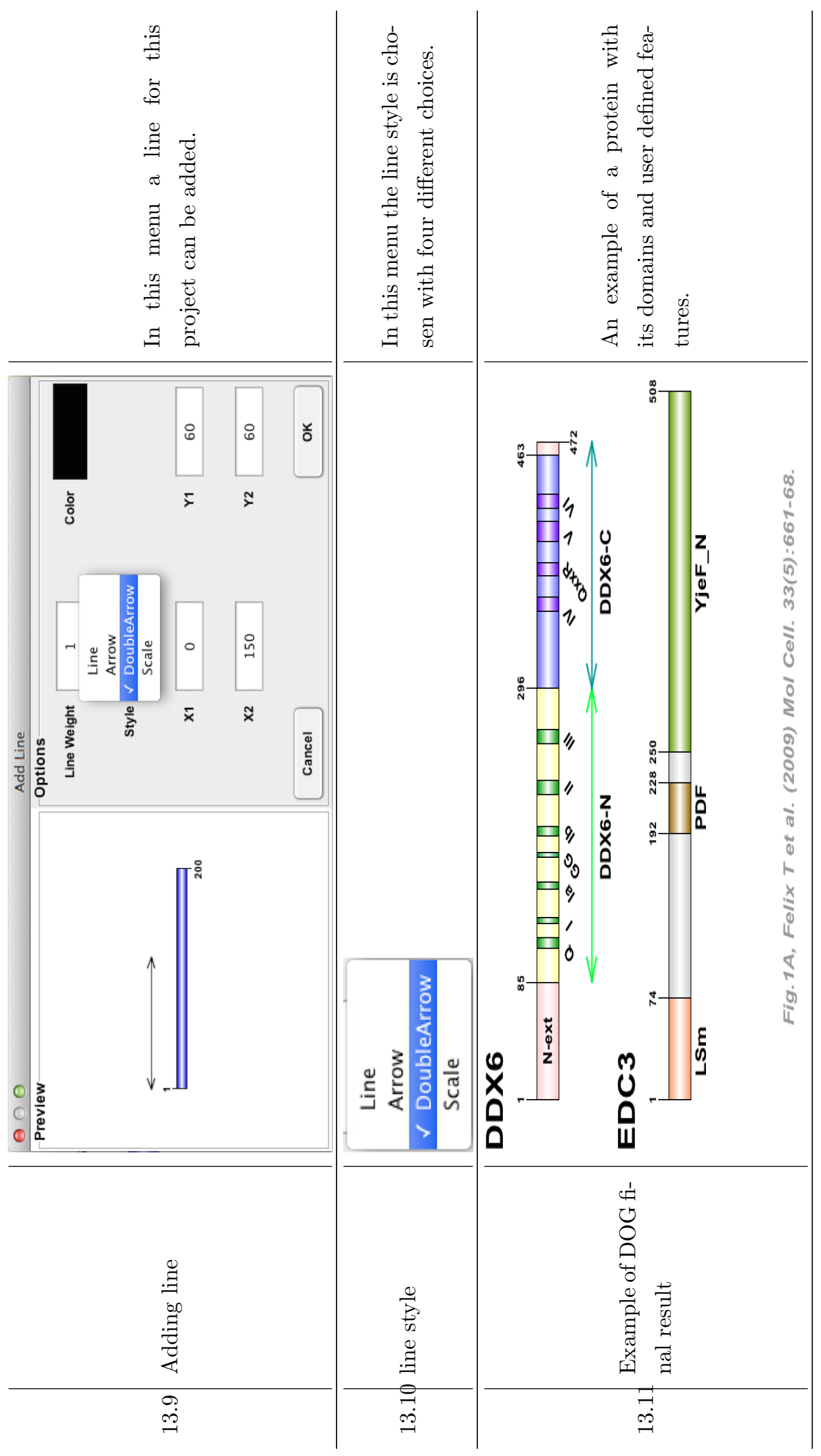




\section{Appendix $\mathrm{C}$}

\section{Comparative Genomics}

Table 14: Comparative Genomics catalog

\begin{tabular}{llll}
\hline $\begin{array}{l}\text { Vis. } \\
\text { Num }\end{array}$ & Vis. Name & Source & Description \\
\hline 141 & Gene tree & http://useast.ensembl.org/inf & $\begin{array}{l}\text { A representative protein for every gene } \\
\text { in Ensembl across all species is used to } \\
\text { determine gene trees and homologues. }\end{array}$ \\
\hline
\end{tabular}

Visualization Sample

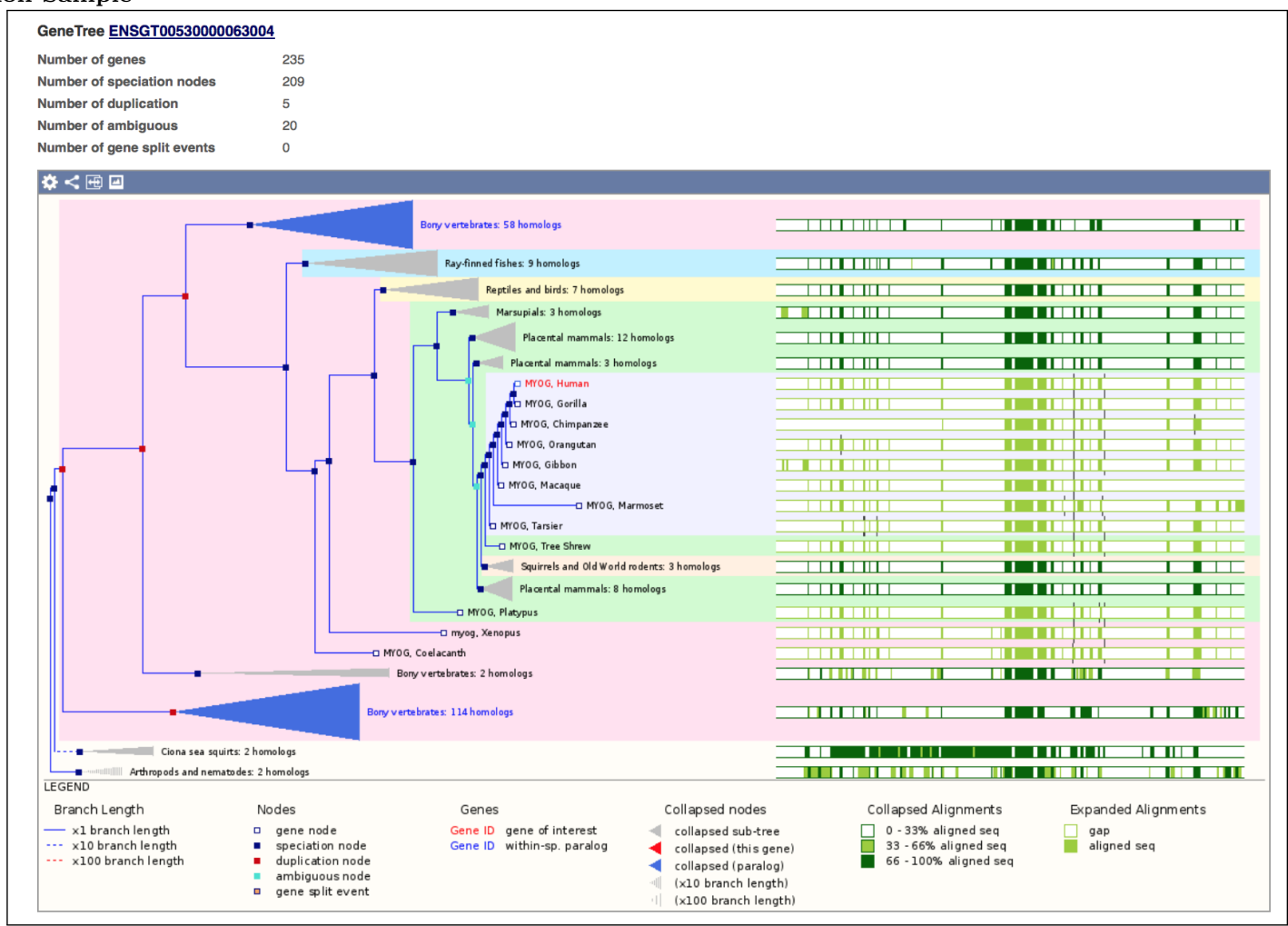




\section{Appendix D}

\section{Glossary}

The glossary is combined from many web-based glossaries. They can be found at :

- http://www.ncbi.nlm.nih.gov/Class/FieldGuide/glossary.html

- http://mousecyc.jax.org/glossary.shtml

- http://www.genome.gov/Glossary/

- www. oxforddictionaries.com/definition/english/glossary

- http://www.biology-online.org/dictionary/Main_Page

- http://smart.embl-heidelberg.de/help/smart_glossary.shtml

- http://www.ncbi.nlm.nih.gov/books/NBK21052/

- http://pfam.xfam.org/help\#tabview=tab9

Accession Number The accession number is the most general identifier used in the NCBI sequence databases. This is the identifier that should be used when citing a database record in a publication. The accession number points to a sequence record and does not change when the sequence is modified. In the Entrez system, using the accession number as a query will retrieve the most recent version of the record. The update history of a particular sequence record is tracked by the accession.version number. Changes in version numbers occur only when the actual sequence of a record has been modified and do not reflect any changes in the annotation. The specific version of a record is also tracked by another identifier that is mainly for internal NCBI use called the GI number.

Active site residues Within an enzyme, a small number of residues are directly involved in the catalysis of a reaction. These are termed active site residues.

Alias An alternative symbol or name for part of the sequence of a known gene that resembles 
names for other anonymous DNA segments. For example, D6Mit236 is an alias for Cftr.

Amino acid An organic acid carrying an amino group. Proteins are linear polymers of the L-forms of 20 common amino acids linked by peptide bonds.

Annotation 1. n. Database entries that provide supplementary information about a biological entity, such as annotation of pathways or regulatory sites. 2. v. The analysis process used to create annotations such as Sequence Annotation.

Associated Genes Associated Genes are genes that have been linked to a gene model by hand curation.

Base pair One of the pairs of chemical bases joined by hydrogen bonds that connect the complementary strands of a DNA molecule or of an RNA molecule that has two strands; the base pairs are adenine with thymine and guanine with cytosine in DNA and adenine with uracil and guanine with cytosine in RNA.

Canonical transcript The canonical transcript is the best representative transcript for a given gene model. Non-canonical: All other transcripts for a gene model that are not the canonical transcript.

cDNA Complementary DNA. A DNA copy of an mRNA or complex sample of mRNAs, made using reverse transcriptase.

CDS Coding sequence; region of nucleotides that corresponds with the sequence of amino acids in a protein (location includes start and stop codons).

Central Dogma The principal statement of the molecular basis of inheritance. In its simplest form:

"DNA makes RNA makes protein."

This means that (generally) genetic information is stored in and transmitted as DNA. Genes are expressed by being copied as RNA (transcription), which is processed into mRNA via splicing and polyadenylation. The information in mRNA is translated into a protein sequence using a genetic code to interpret three-base codons as instructions to add one of twenty amino acids or to stop translation.

Codon Three bases in a DNA or RNA sequence that specify an amino acid or a termination signal (stop codon).

Coiled-coils Coiled coils are motifs found in proteins that structurally form alpha-helices that wrap or wind around each other. Normally, two to three helices are involved, but cases of up to seven alpha-helices have been reported. Coilded-coil are found in a wide variety of proteins, many functionally very important.

Domain A domain is a discrete structural unit of a protein. In principle, protein domains are capable of folding independently from the rest of the protein. Domains can often be identified 
by non-structural approaches based on conserved amino acid sequences. The NCBI's CDDsearch uses information from curated multiple sequence alignments to identify domains in protein sequences.

Domain composition Proteins with the same domain composition have at least one copy of each of domains of the query.

Ensembl Ensembl is a joint project between EBI-EMBL and the Sanger Institute to provide automatic annotation of eukaryotic genomes.

Entrez Entrez is an integrated search and retrieval system that integrates information from various databases at NCBI, including nucleotide and protein sequences, 3D structures and structural domains, genomes, variation data (SNPs), gene expression data, genetic mapping data, population studies, OMIM, taxonomy, books online, and the biomedical literature.

Essential amino acid Essential amino acids cannot be made by the body. As a result, they must come from food. The nine essential amino acids are: histidine, isoleucine, leucine, lysine, methionine, phenylalanine, threonine, tryptophan, and valine.

Eukaryote A cell or organism with membrane-bound, structurally discrete nucleus and other welldeveloped sub-cellular compartments.

Evidence Type The source of evidence to support the gene model.

Exon Part of a gene whose sequence is present in a mature mRNA after splicing.

Expect Value (E-value) In BLAST statistics, the Expect value is the number of alignments with a particular score, or a better score, that are expected to occur by chance when comparing two random sequences.

Gene The fundamental unit of inheritance, comprising a segment of DNA (or RNA in some viruses) that codes for one or several related functions and occupies a fixed position (locus) on a chromosome. "proteins coded directly by genes". (In technical use) a distinct sequence of nucleotides forming part of a chromosome, the order of which determines the order of monomers in a polypeptide or nucleic acid molecule which a cell (or virus) may synthesize.

Gene expression The conversion of the information from the gene into mRNA via transcription and then to protein via translation resulting in the phenotypic manifestation of the gene.

Gene Model A representation of a gene that contains information about features of the transcript such as exon- intron boundaries, splice sites, UTRs, etc. A gene model is a mapping of gene features such as coding regions and exon intron boundaries onto the the genomic DNA of an organism. Gene models typically provide a predicted transcript and protein sequence. A simple kind of gene model can be made by aligning an expressed sequence (cDNA) to the genomic DNA sequence. More precise exon intron boundaries can be identified by constraining the aligned segments using consensus splicing signals. 
Gene product A gene product is any protein or enzyme that occurs as a result of translation of a gene. A gene, then, refers to a particular piece of DNA that, in genetic code, contains the recipe for a gene product.

Gene products are the actual materials that carry on the daily functions of life, such as digesting foods, making new living tissue, and converting materials into energy; actions that virtually all living things undergo in life.

Intron Part of a gene whose sequence is transcribed but not present in a mature mRNA after splicing.

Locus Literally, "place". The location of a gene or set of genes on a chromosome.

Low complexity regions Low complexity regions are regions of biased sequence composition, usually comprised of different types of repeats. These regions have been shown to be functionally important in some proteins, but they are generally not well understood and are masked out to focus on globular domains within the protein.

Motif A motif is a short, well-conserved nucleotide or amino acid sequence that represents a minimal functional domain. It is often a consensus for several aligned sequences. The PROSITE database is a popular collection of protein motifs, including motifs for enzyme catalytic sites, prosthetic group attachment sites (heme, biotin, etc), and regions involved in binding another protein. Examples of DNA motifs are transcription factor binding sites.

mRNA Messenger RNA. An RNA molecule that is the product of transcription of a gene. In eukaryotes, that molecule often has to be spliced and polyadenylated before it can be translated into a protein product.

Mutation 1. The process through which genes undergo a structural change.

2. Any permanent change in DNA, i.e., in its nucleotide sequence. Examples include chromosome rearrangements and point mutations.

Nonessential amino acid An amino acid that can be synthesized in the body and does not have to be obtained from the diet.

Open Reading Frame (ORF) 1. A length of nucleotide sequence that lacks termination codons in a given reading frame.

2. An open reading frame is a portion of a DNA molecule that, when translated into amino acids, contains no stop codons. The genetic code reads DNA sequences in groups of three base pairs, which means that a double-stranded DNA molecule can read in any of six possible reading frames - three in the forward direction and three in the reverse. A long open reading frame is likely part of a gene.

Orthologue Orthologues are genes derived from a common ancestor through vertical descent. This is often stated as the same gene in different species. In contrast, paralogs are genes within the same genome that have evolved by duplication. 
Paralog Paralogs are usually described as genes within the same genome that have evolved by duplication.

PFAM Pfam is a database of protein domain families represented as (i) multiple alignments, and (ii) HMM-profiles.

Prokaryote A microscopic single-celled organism that has neither a distinct nucleus with a membrane nor other specialized organelles. Prokaryotes include the bacteria and cyanobacteria.

Protein A large complex molecule made up of one or more chains of amino acids joined by peptide bonds. Proteins are the principal constituents of cellular material and serve as enzymes, hormones, structural elements, and antibodies; they are synthesized in the body from their constituent amino acids.

Protein folding "Protein folding is the process by which a protein structure assumes its functional shape or conformation. It is the physical process by which a polypeptide folds into its characteristic and functional three-dimensional structure from random coil. Each protein exists as an unfolded polypeptide or random coil when translated from a sequence of mRNA to a linear chain of amino acids. This polypeptide lacks any stable (long-lasting) three-dimensional structure. Amino acids interact with each other to produce a well-defined three-dimensional structure, the folded protein (the right hand side of the figure), known as the native state. The resulting three-dimensional structure is determined by the amino acid sequence (Anfinsen's dogma). Experiments beginning in the 1980s indicate the codon for an amino acid can also influence protein structure. Source: http://en.wikipedia.org/wiki/Protein_folding.

Sequence Alignment A sequence alignment is a residue by residue comparison of two or more sequences. In the alignment, the relative positions of the sequences are adjusted to optimize (usually maximize) the alignment score derived by reference to some scoring matrix. In some cases gaps with associated penalties may be inserted into one or more sequences to optimize the alignment score.

Sequence Annotation 1. n. Additional information added to genomic sequence to identify genes, delimit the intron and exon structures of those genes, identify regulatory elements, note the positions of allelic variation, etc. 2. v. The analysis process used to create sequence annotations. The process relies heavily on the homology principle, whereby similarity to known genes is used to help identify new genes and propose functions for them.

Sequence ID (SeqID) Sequence accession identifier. A unique alphanumeric character string that unambiguously identifies a sequence record in a database. Examples of genomic sequence providers are NCBI and Ensembl; examples of sequence IDs from these providers are 16590 and ENSMUSG00000053869, respectively.

Signal peptides Signal peptides are short regions ( $<60$ residues long) found at the N-terminus of proteins, which direct the post-translational transport of a protein and are subsequently 
removed by peptidases. More specifically, a signal peptide is characterized by a short hydrophobic helix (approximately 7-15 residues). This helix is preceded by a slight positively charged region of highly variable length (approximately 1-12 residues). Between the hydrophobic helix and the cleavage site is a somewhat polar and uncharged region, of between 3 and 8 amino-acids.

SMART SMART (Simple Modular Architecture Retrieval Tool) is a database of conserved domains that allows automatic identification and annotation of domains in user-supplied protein sequences. Th SMART data are used create one of the sets of PSSMs used in the CD-Search.

Splice In genetics: splice means Join or insert (a gene or gene fragment): "they have spliced a gene into tomatoes that improves flavour"

Splicing Part of the processing of an RNA transcript into mRNA, in which introns are removed enzymatically.

Strain Strain is a low-level taxonomic rank used in three related ways. In Microbiology, a strain is a genetic variant or subtype of a microorganism (e.g. virus or bacterium or fungus). In plants, a strain is a designated group of offspring that have descended from a modified plant, produced either by conventional breeding or by biotechnological means or result from genetic mutation. In rodents, a strain is a group of animals that is genetically uniform.

Stop Codon A stop codon is a trinucleotide sequence within a messenger RNA (mRNA) molecule that signals a halt to protein synthesis. The genetic code describes the relationship between the sequence of DNA bases (A, C, G, and T) in a gene and the corresponding protein sequence that it encodes. The cell reads the sequence of the gene in groups of three bases. Of the 64 possible combinations of three bases, 61 specify an amino acid, while the remaining three combinations are stop codons.

Structured Data Structured data are data that have been represented in a manner that allows computation with those data. Data become structured when they are carefully dissected and assigned to distinct fields of a database with clearly defined meanings, so that the data are independently queryable and computable. Therefore, we can ask questions across the data such as "find all enzymes that use magnesium as a cofactor" or "find all pathways in which pyruvate is an input substrate".

Structural Gene A gene that encodes an enzyme or structural protein, in contrast to a regulatory gene.

Structural Protein A protein that functions as a structural element of cells rather than as an enzyme, for example, collagen.

STS Sequence Tagged Site. A short segment of unique sequence derived from genomic DNA. A large collection of STSs can be used to assemble a physical map of the genome from a collection of genomic clones (e.g., BACs or YACs) by testing each clone for the presence of each STS. 
Two clones that contain one or more STSs in common must overlap. For examples, see the physical maps of the mouse genome at MGI.

Synthesize (chemistry, biochemistry) To produce substance by combining chemical precursors.

Tab-delimited file A text file that uses tabs to separate adjacent fields. It is a common format for downloading information into a spreadsheet.

Taxon A stable unique identification number for the taxon of the source oganism. A taxonomy ID number is assigned to each taxon (species, genus, family, etc.) in the NCBI Taxonomy Database. See also the Organism field, above.

Transcription The enzymatic synthesis of an RNA molecule directed by information in a DNA molecule.

Translation The enzymatic synthesis of a protein molecule directed by the information in an mRNA molecule. The mRNA is read from the 5' end to the 3 ' end, with the protein being synthesized from the amino terminus to the carboxyl terminus.

Transmembrane domain Transmembrane domain usually denotes a single transmembrane alpha helix of a transmembrane protein. Also known as an integral protein.

Transmembrane protein Membrane protein that extends through the lipid bilayer, with part of its mass on either side of the membrane.

tRNA Transfer RNA. Small RNA molecules that bind to the codons of mRNA in the ribosome after being "charged" with amino acids.

Visualization idiom is a specific sequence of data enrichment and enhancement transformations, visualization mappings and rendering transformations that produce an abstract display of a scientific data set.

X Chromosome One of pair of chromosomes that is sexually dimorphic in mammals. Normal female mammals have two $\mathrm{X}$ chromosomes, while normal male mammals have an $\mathrm{X}$ chromosome and a $\mathrm{Y}$ chromosome.

Y Chromosome One of pair of chromosomes that is sexually dimorphic in mammals. Normal female mammals have two $\mathrm{X}$ chromosomes, while normal male mammals have an $\mathrm{X}$ chromosome and a $\mathrm{Y}$ chromosome.

3' (3-prime) A term that identifies one end of a single-stranded nucleic acid molecule. The 3' end is that end of the molecule which terminates in a 3' phosphate group. The 3' direction is the direction toward the 3' end. Nucleic acid sequences are written with the 5' end to the left and the 3' end to the right, in reference to the direction of DNA synthesis during replication (from 5' to 3'), RNA synthesis during transcription (from 5' to 3'), and the reading of mRNA sequence (from 5' to 3') during translation. 
3' UTR 3' Untranslated Region. That portion of an mRNA from the 3' end to the position of the last codon used in translation.

5' (5-prime) A term that identifies one end of a single-stranded nucleic acid molecule. The 5' end is that end of the molecule which terminates in a 5' phosphate group. The $5^{\prime}$ direction is the direction toward the 5' end. Nucleic acid sequences are written with the 5' end to the left and the 3' end to the right, in reference to the direction of DNA synthesis during replication (from 5' to 3'), RNA synthesis during transcription (from 5' to 3'), and the reading of mRNA sequence (from 5' to 3') during translation.

5' UTR 5' Untranslated Region. That portion of an mRNA from the 5' end to the position of the first codon used in translation. 